\title{
Modes of micromolar host-guest binding of $\beta$-cyclodextrin complexes revealed by NMR spectroscopy in salt water
}

\author{
Supplementary Information
}

Josef Tomeček, ${ }^{a}$ Andrea Čablová, ${ }^{a}$ Aneta Hromádková, ${ }^{a}$ Jan Novotný, ${ }^{* b, c, d}$ Radek Marek, ${ }^{\text {b,c,d }}$ Ivo Durník, ${ }^{\text {b,c }}$ Petr Kulhánek, ${ }^{* b, c}$ Zdeňka Prucková, ${ }^{a}$ Michal Rouchal, ${ }^{a}$ Lenka Dastychová, ${ }^{\text {a }}$ and Robert Vícha*a

a Department of Chemistry, Faculty of Technology, Tomas Bata University in Zlín, Vavrečkova 275, 760 01 Zlín, Czech Republic. E-mail: rvicha@utb.cz; Tel: +420-576031103

${ }^{\mathrm{b}}$ CEITEC - Central European Institute of Technology, Masaryk University, Kamenice 5, 62500 Brno, Czech Republic. E-mail: kulhanek@chemi.muni.cz; Tel: +420-549495459 (P. Kulhánek). E-mail: jan.novotny@ceitec.muni.cz; Tel: +420-549496440 (J. Novotný).

${ }^{\mathrm{c}}$ National Centre for Biomolecular Research, Faculty of Science, Masaryk University, Kamenice 5, 62500 Brno, Czech Republic.

${ }^{\mathrm{d}}$ Department of Chemistry, Faculty of Science, Masaryk University, Kamenice 5, 62500 Brno, Czech Republic.

\section{Table of Contents}

${ }^{1} \mathrm{H}$ and ${ }^{13} \mathrm{C}\left\{{ }^{1} \mathrm{H}\right\}$ NMR spectra of compounds $\mathbf{4 a}, \mathbf{4 b}, \mathbf{5 a}, \mathbf{5 b}, \mathbf{8}-\mathbf{1 2}$

Stacking plots of NMR titrations

ITC data for the guests $\mathbf{5 a}, \mathbf{5 b}, \mathbf{1 0}-\mathbf{1 2}$

Crystal data and structure refinement of compounds $\mathbf{3 b}, \mathbf{5 b}$

Atomic coordinates, geometrical parameters and atomic displacement parameters

ESI-MS spectra of free guests

ESI-MS spectra of complexes

Computational details 
${ }^{1} \mathrm{H}$ and ${ }^{13} \mathrm{C}\left\{{ }^{1} \mathrm{H}\right\}$ NMR spectra of compounds $4 \mathrm{a}, 4 \mathrm{~b}, 5 \mathrm{a}, 5 \mathrm{~b}, 8,9,10,11,12$

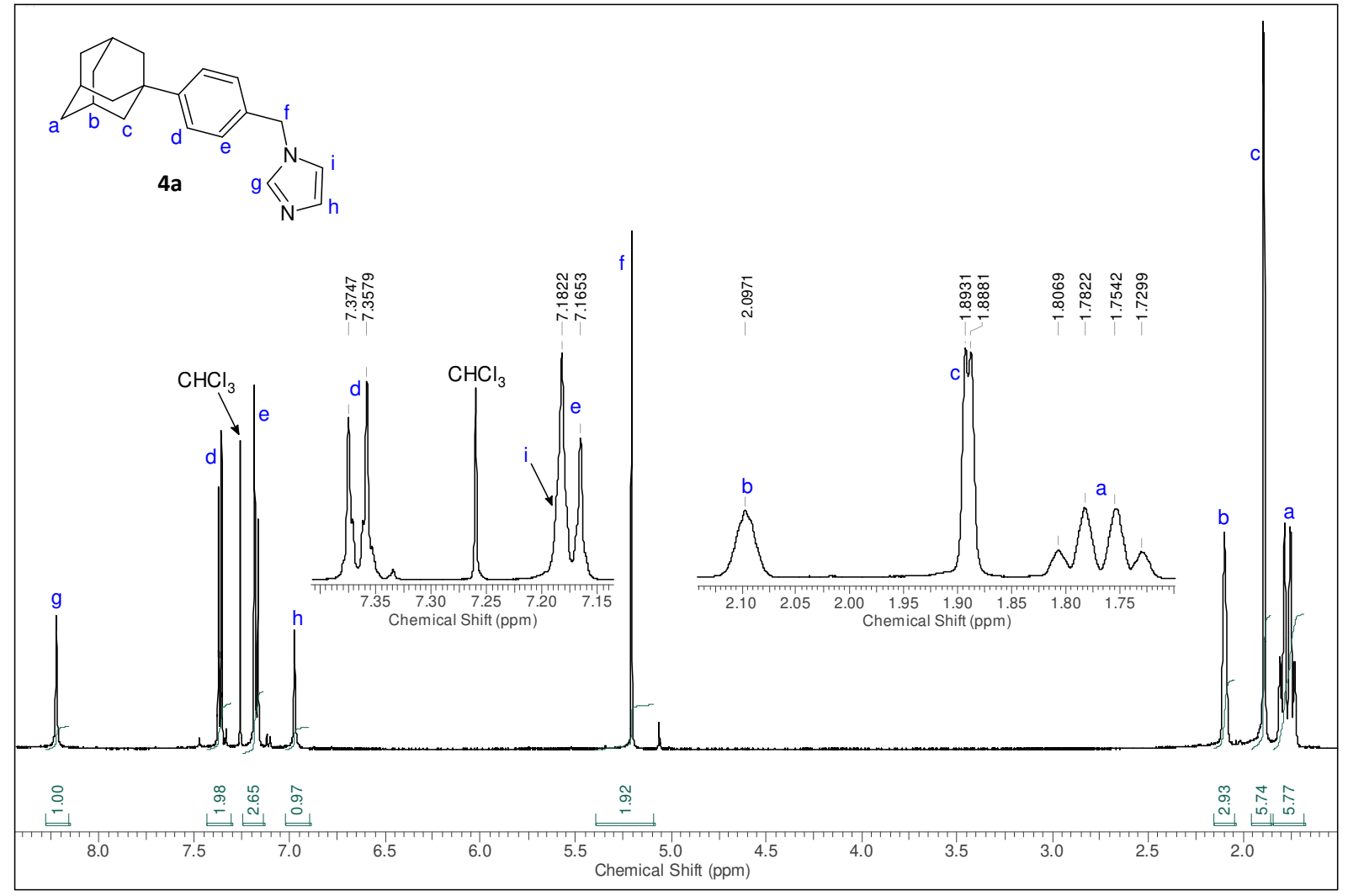

Figure $\mathbf{S 1}$ The ${ }^{1} \mathrm{H}$ NMR $\left(\mathrm{CDCl}_{3}, 500 \mathrm{MHz}\right)$ spectrum of compound $\mathbf{4 a}$.

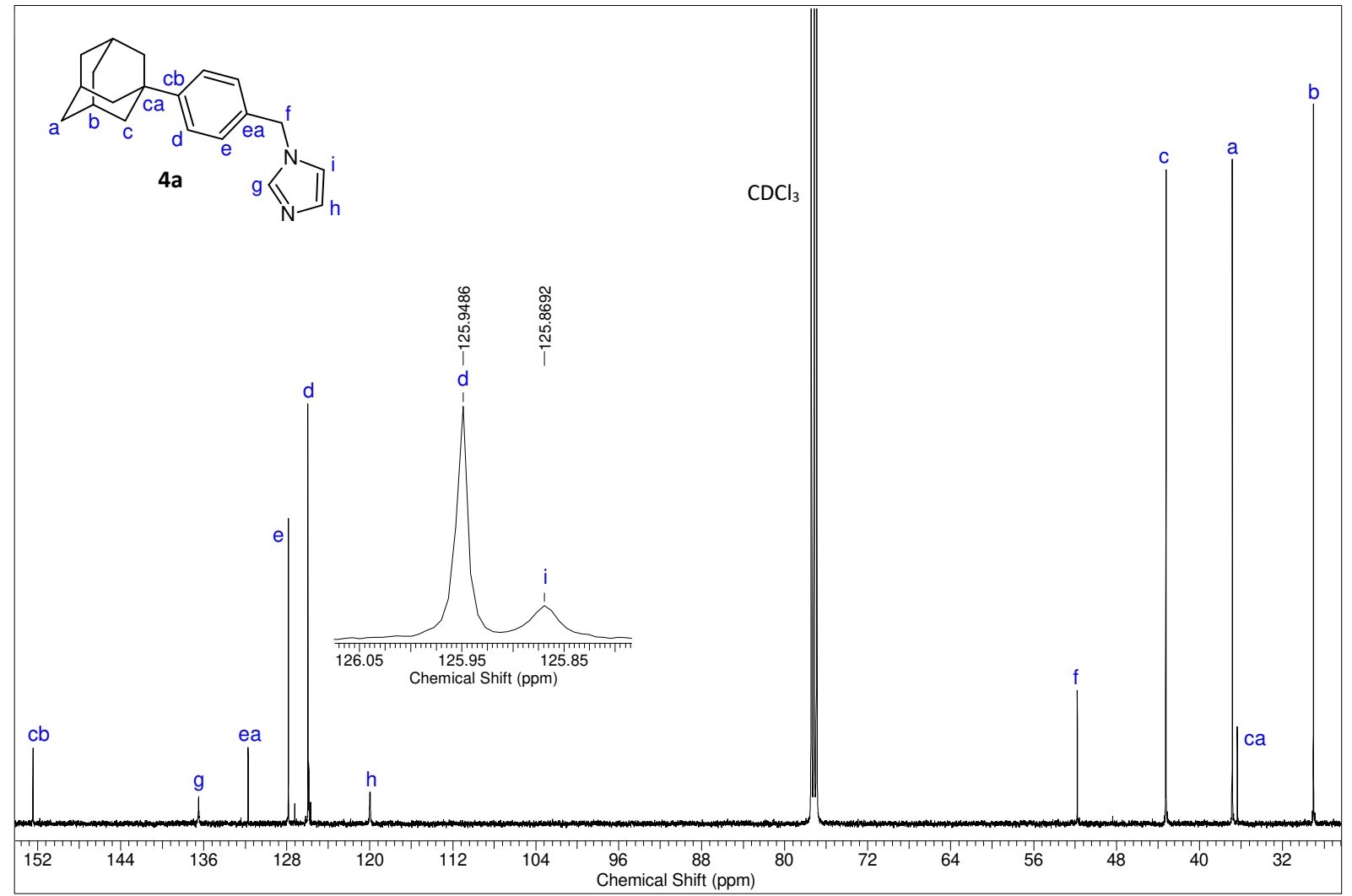

Figure S2 The ${ }^{13} \mathrm{C}\left\{{ }^{1} \mathrm{H}\right\}$ NMR $\left(\mathrm{CDCl}_{3}, 126 \mathrm{MHz}\right)$ spectrum of compound $\mathbf{4 a}$. 


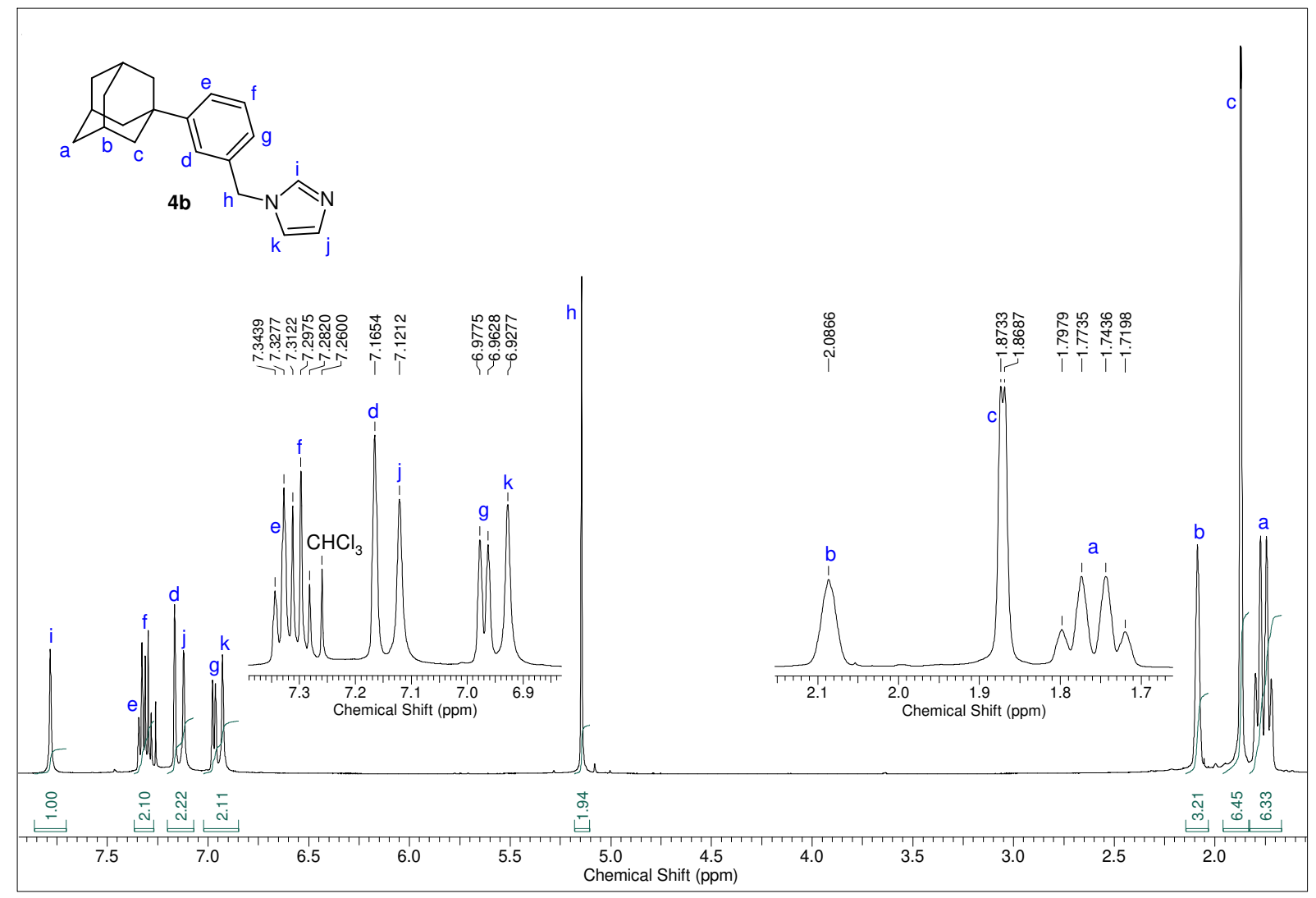

Figure $\mathbf{S 3}$ The ${ }^{1} \mathrm{H} \mathrm{NMR}\left(\mathrm{CDCl}_{3}, 500 \mathrm{MHz}\right)$ spectrum of compound $\mathbf{4 b}$.

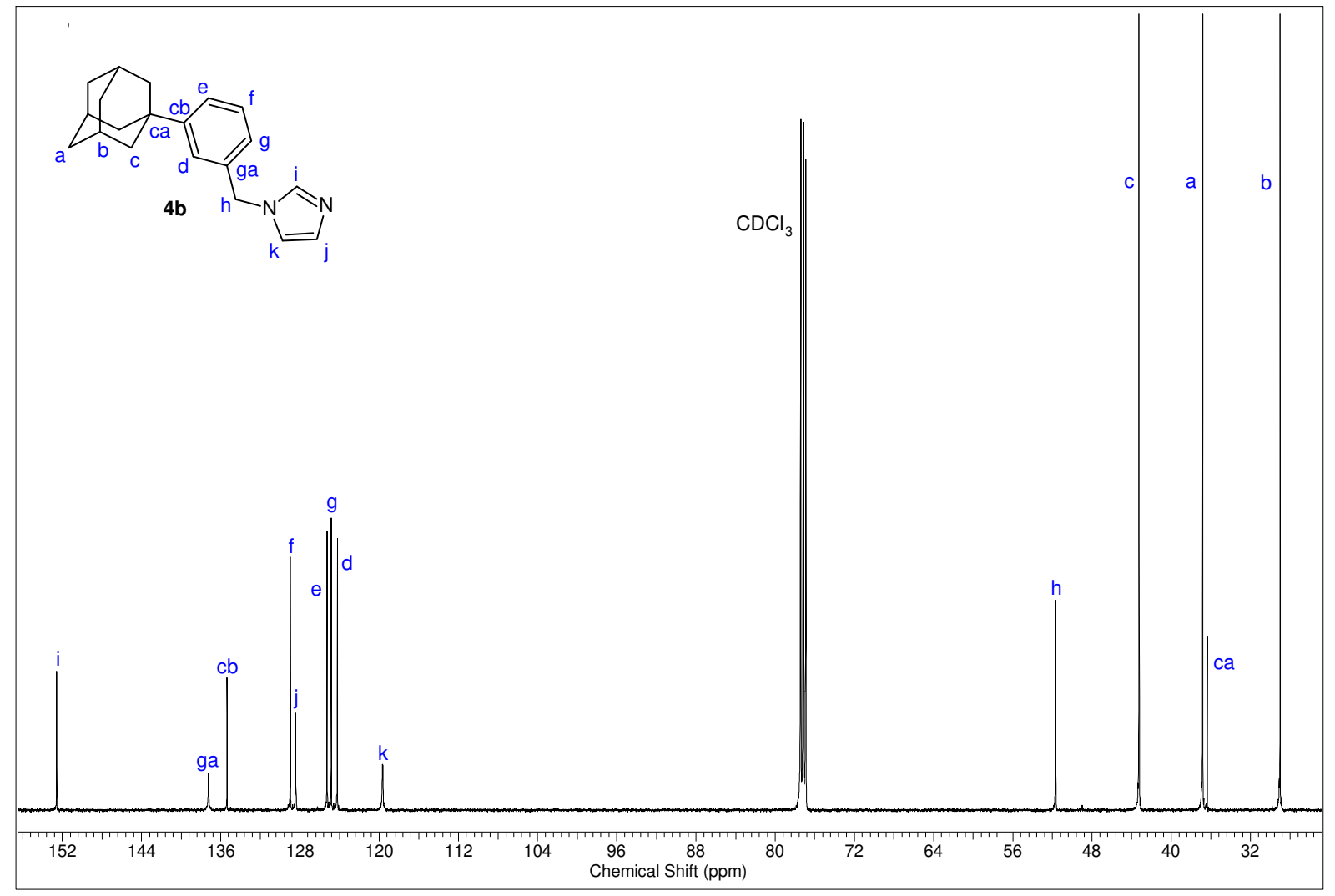

Figure $\mathbf{S 4}$ The ${ }^{13} \mathrm{C}\left\{{ }^{1} \mathrm{H}\right\}$ NMR $\left(\mathrm{CDCl}_{3}, 126 \mathrm{MHz}\right)$ spectrum of compound $\mathbf{4 b}$. 


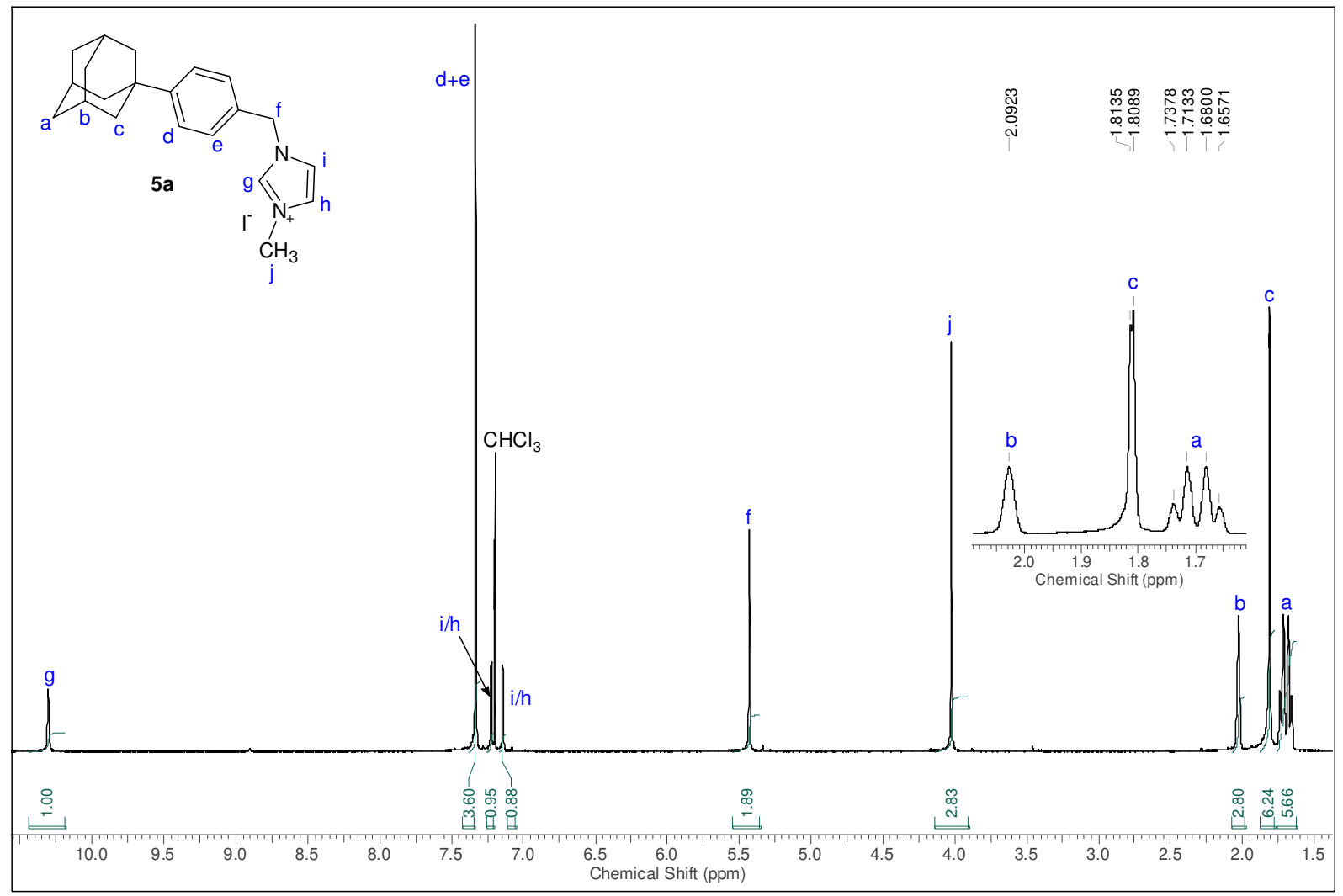

Figure $\mathbf{S 5}$ The ${ }^{1} \mathrm{H}$ NMR $\left(\mathrm{CDCl}_{3}, 500 \mathrm{MHz}\right)$ spectrum of compound $\mathbf{5 a}$.

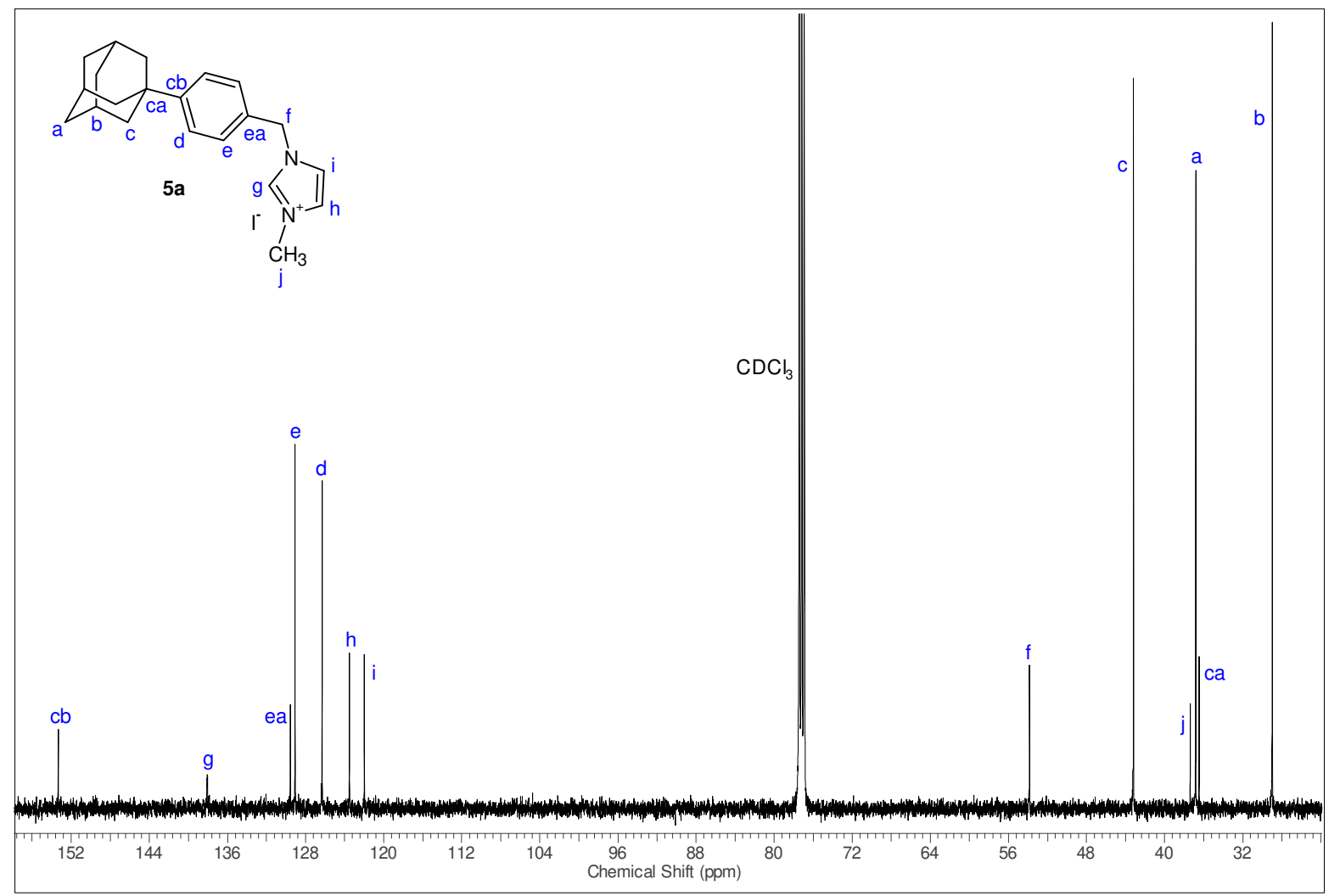

Figure S6 The ${ }^{13} \mathrm{C}\left\{{ }^{1} \mathrm{H}\right\}$ NMR $\left(\mathrm{CDCl}_{3}, 126 \mathrm{MHz}\right)$ spectrum of compound $5 \mathbf{a}$. 


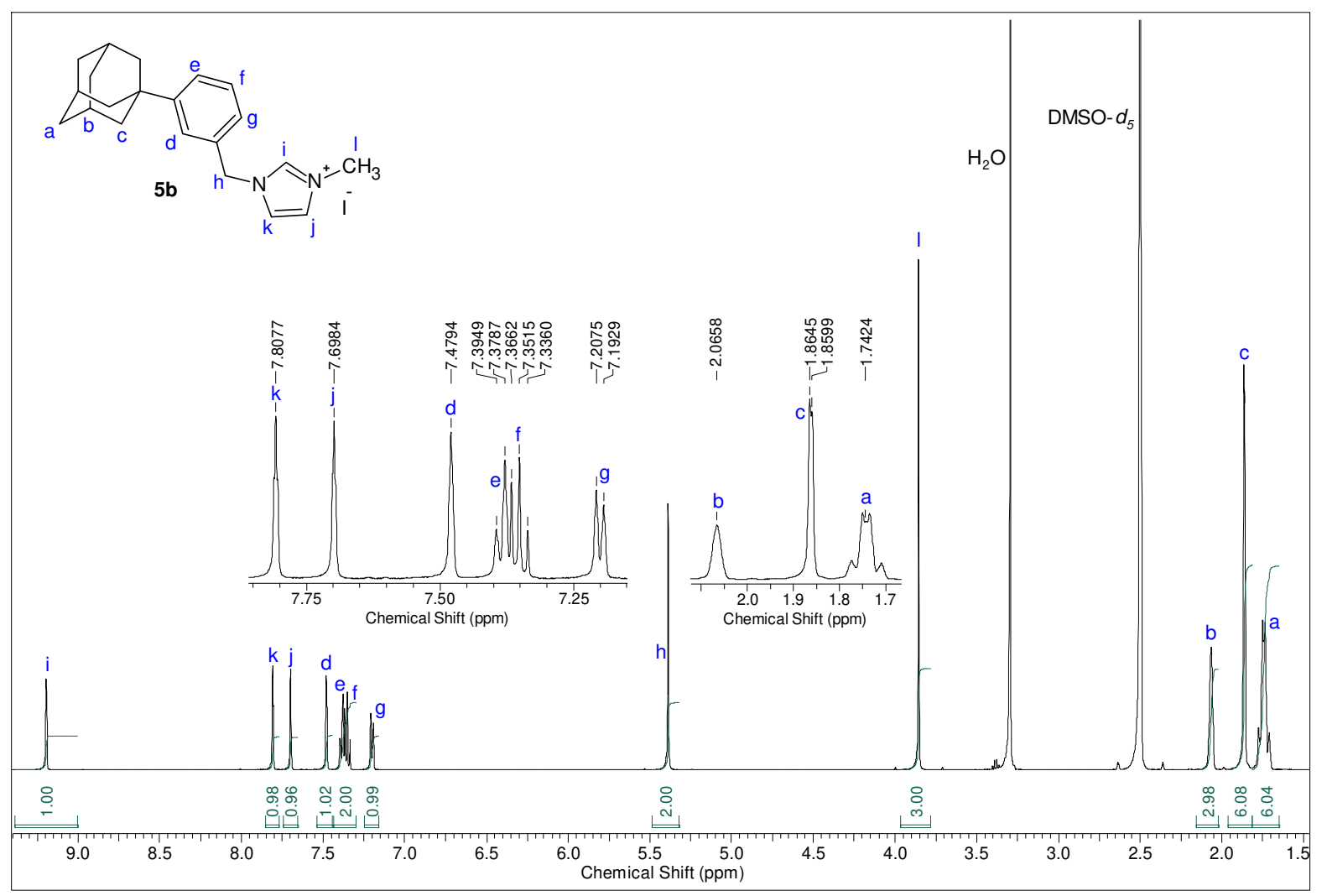

Figure S7 The ${ }^{1} \mathrm{H}$ NMR (DMSO- $d_{6}, 500 \mathrm{MHz}$ ) spectrum of compound $\mathbf{5 b}$.

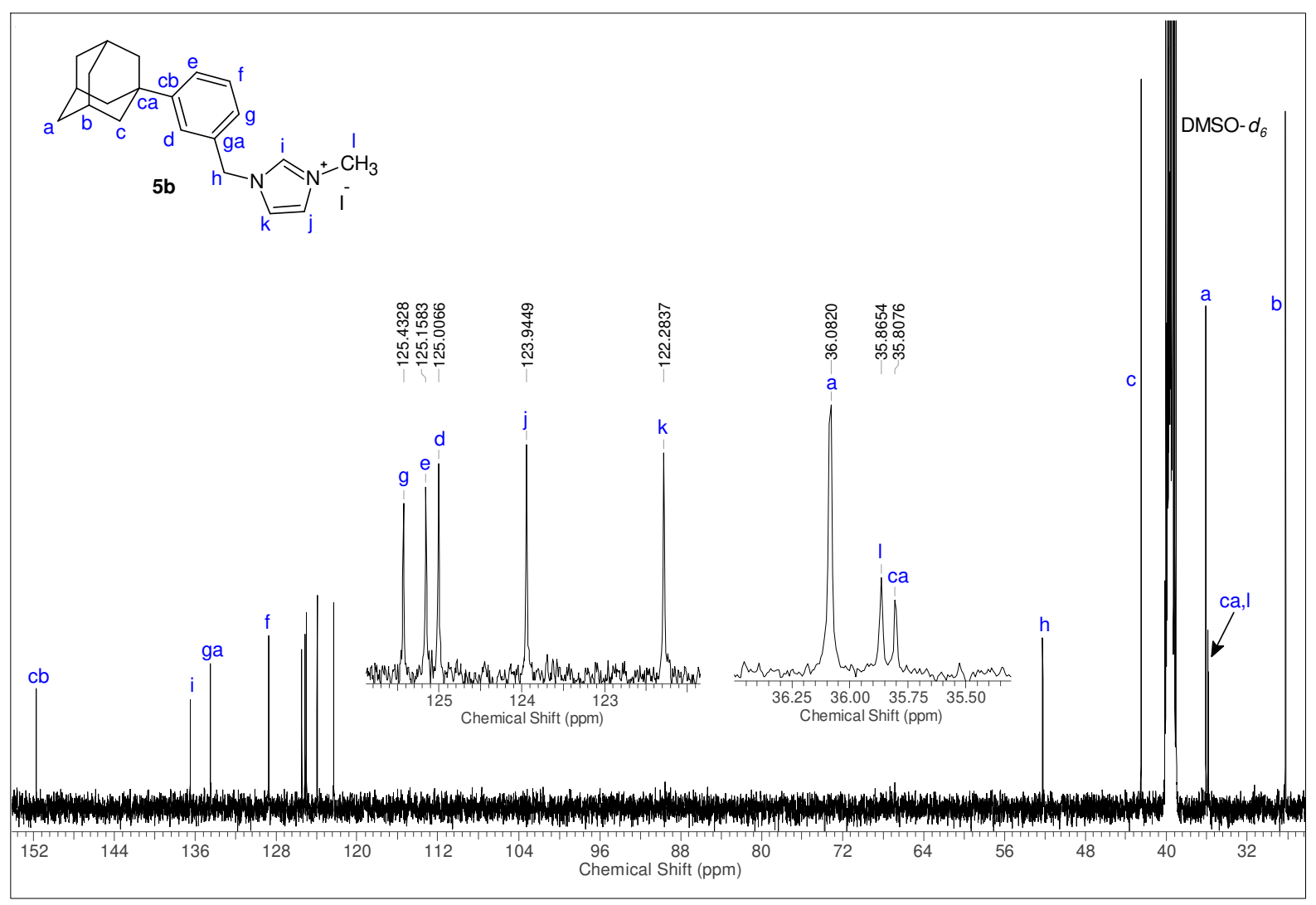

Figure S8 The ${ }^{13} \mathrm{C}\left\{{ }^{1} \mathrm{H}\right\}$ NMR (DMSO- $d_{6}, 126 \mathrm{MHz}$ ) spectrum of compound $\mathbf{5 b}$. 


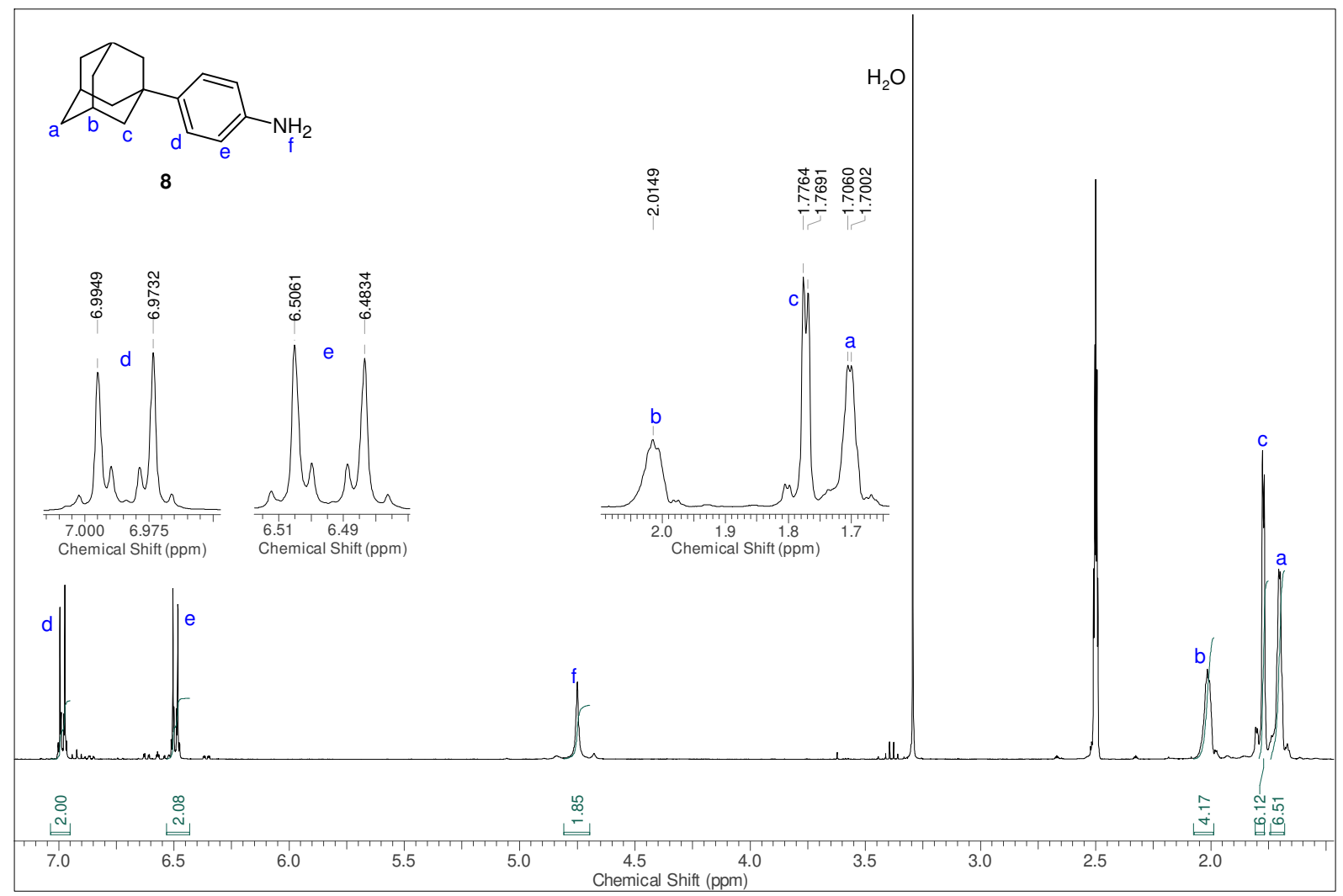

Figure S9 The ${ }^{1} \mathrm{H}$ NMR (DMSO- $d_{6}, 400 \mathrm{MHz}$ ) spectrum of compound 8 .

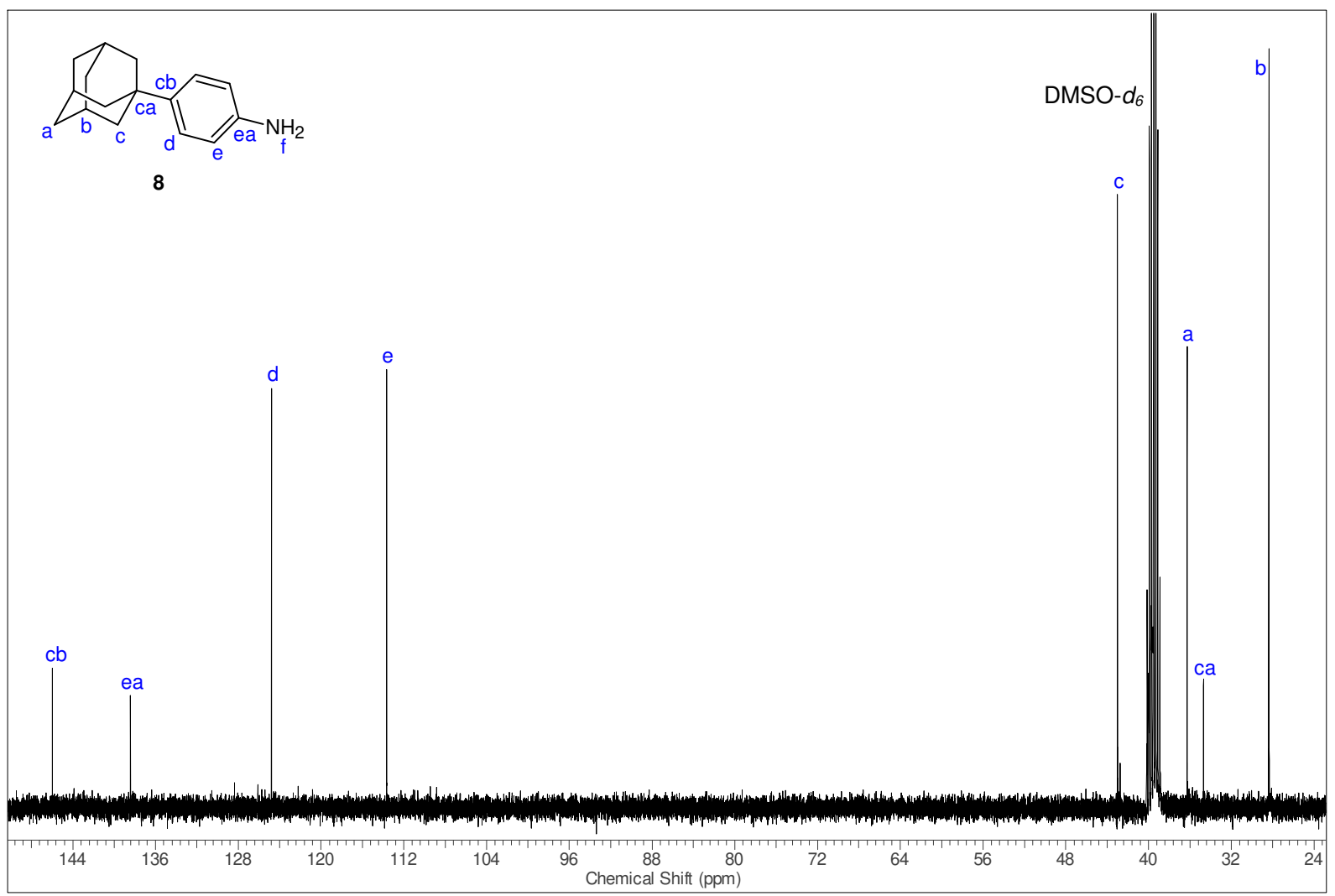

Figure S10 The ${ }^{13} \mathrm{C}\left\{{ }^{1} \mathrm{H}\right\}$ NMR (DMSO- $d_{6}, 101 \mathrm{MHz}$ ) spectrum of compound 8. 


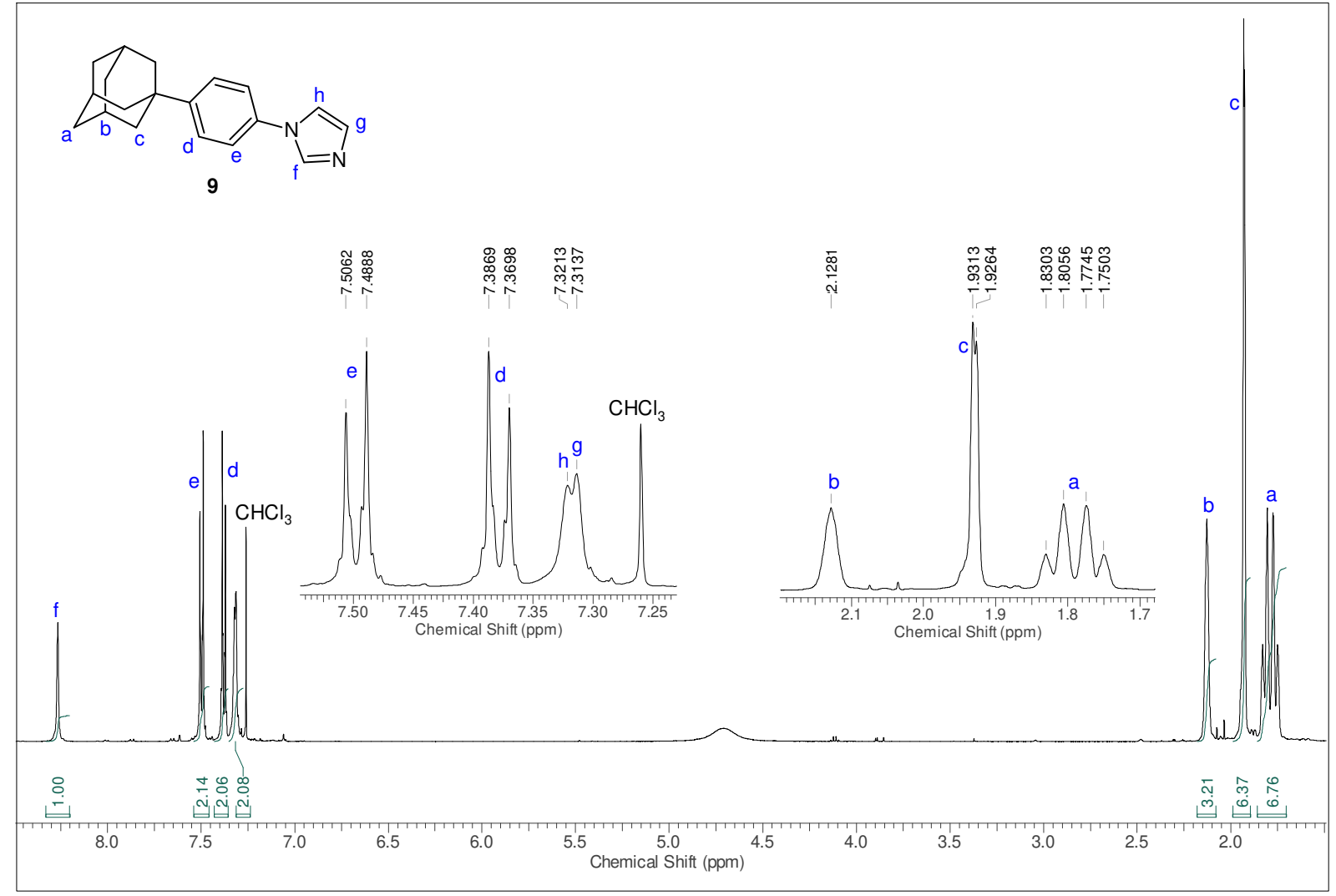

Figure S11 The ${ }^{1} \mathrm{H}$ NMR $\left(\mathrm{CDCl}_{3}, 500 \mathrm{MHz}\right)$ spectrum of compound 9.

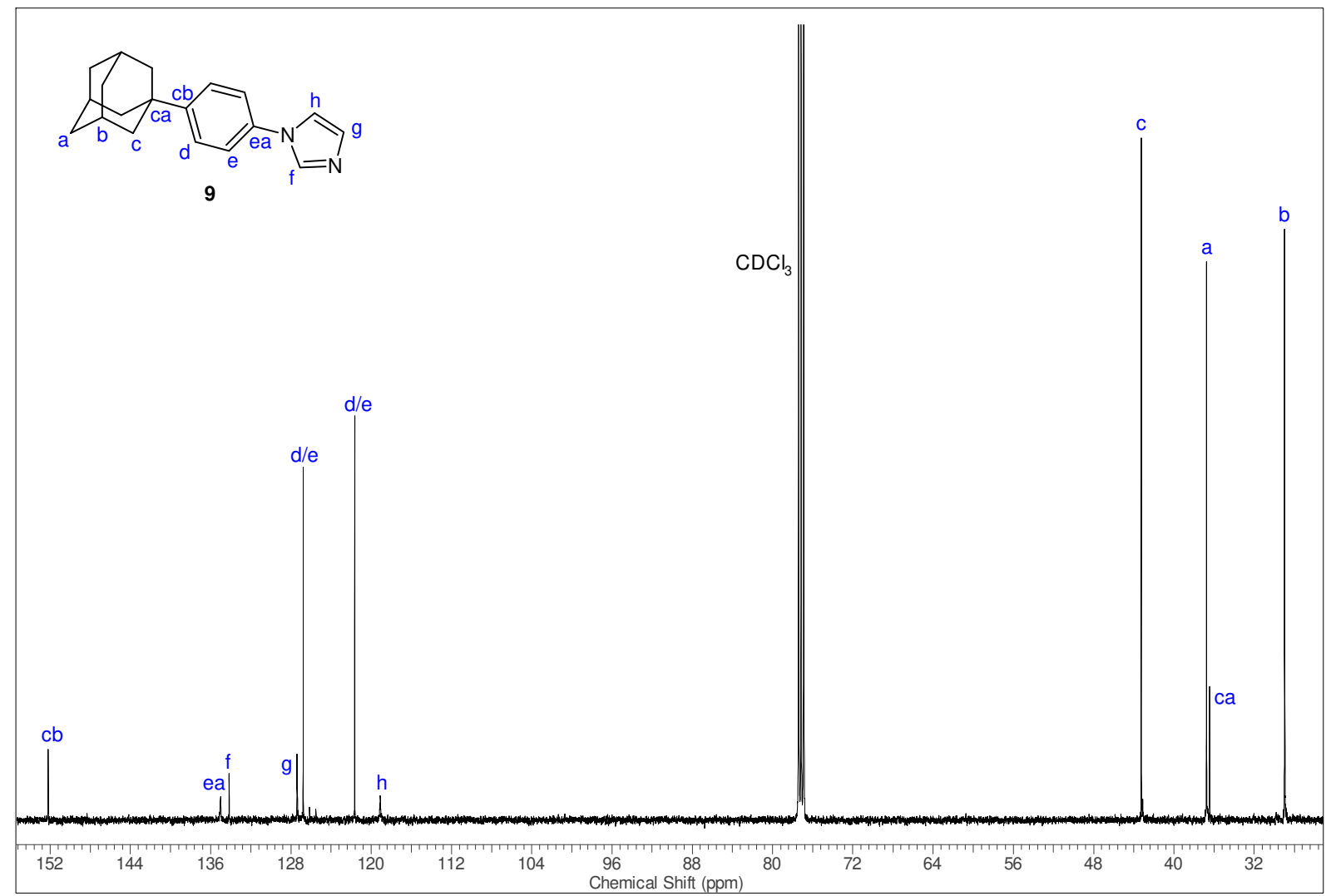

Figure S12 The ${ }^{13} \mathrm{C}\left\{{ }^{1} \mathrm{H}\right\}$ NMR $\left(\mathrm{CDCl}_{3}, 126 \mathrm{MHz}\right)$ spectrum of compound 9. 


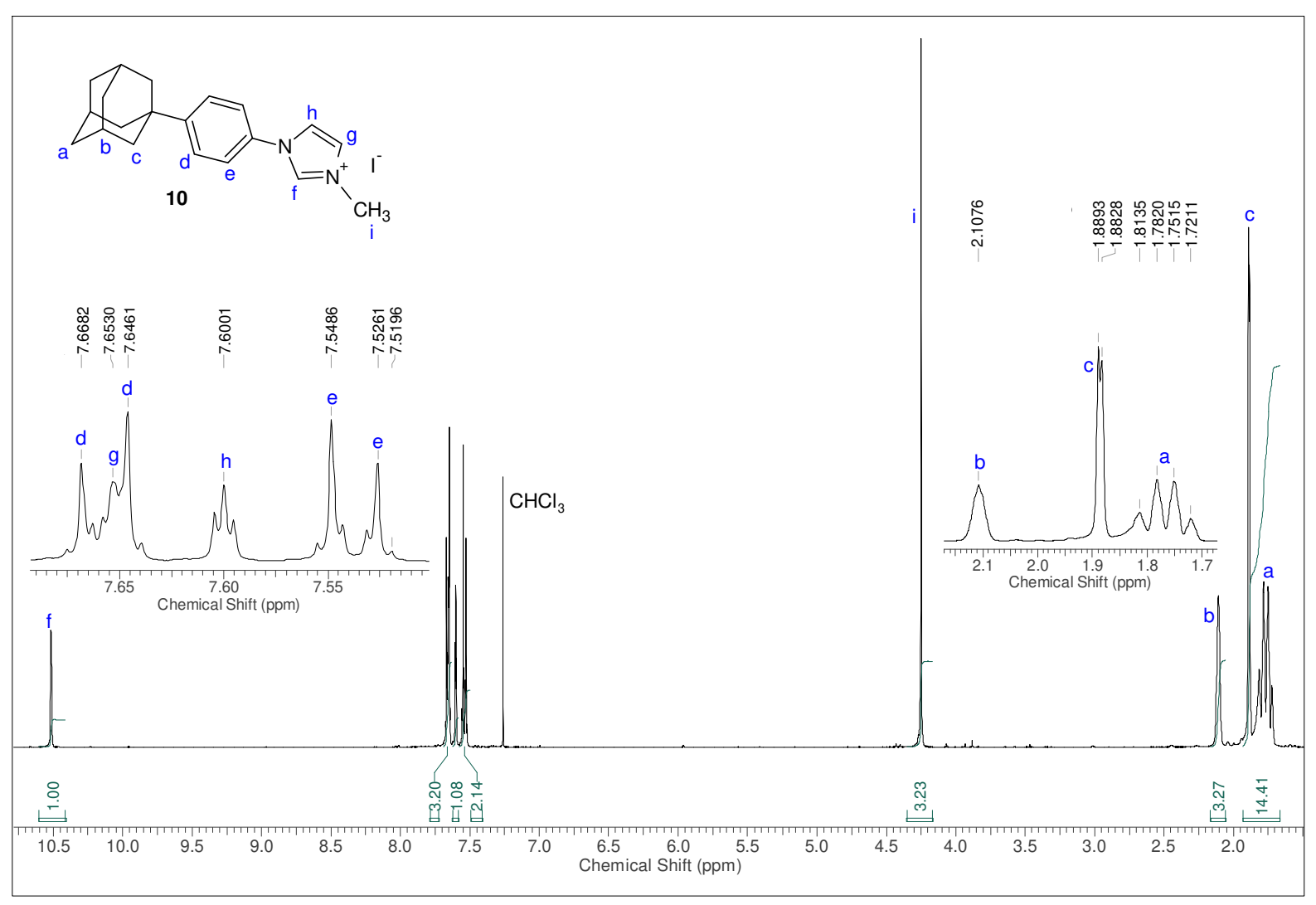

Figure S13 The ${ }^{1} \mathrm{H}$ NMR $\left(\mathrm{CDCl}_{3}, 500 \mathrm{MHz}\right)$ spectrum of compound $\mathbf{1 0}$.

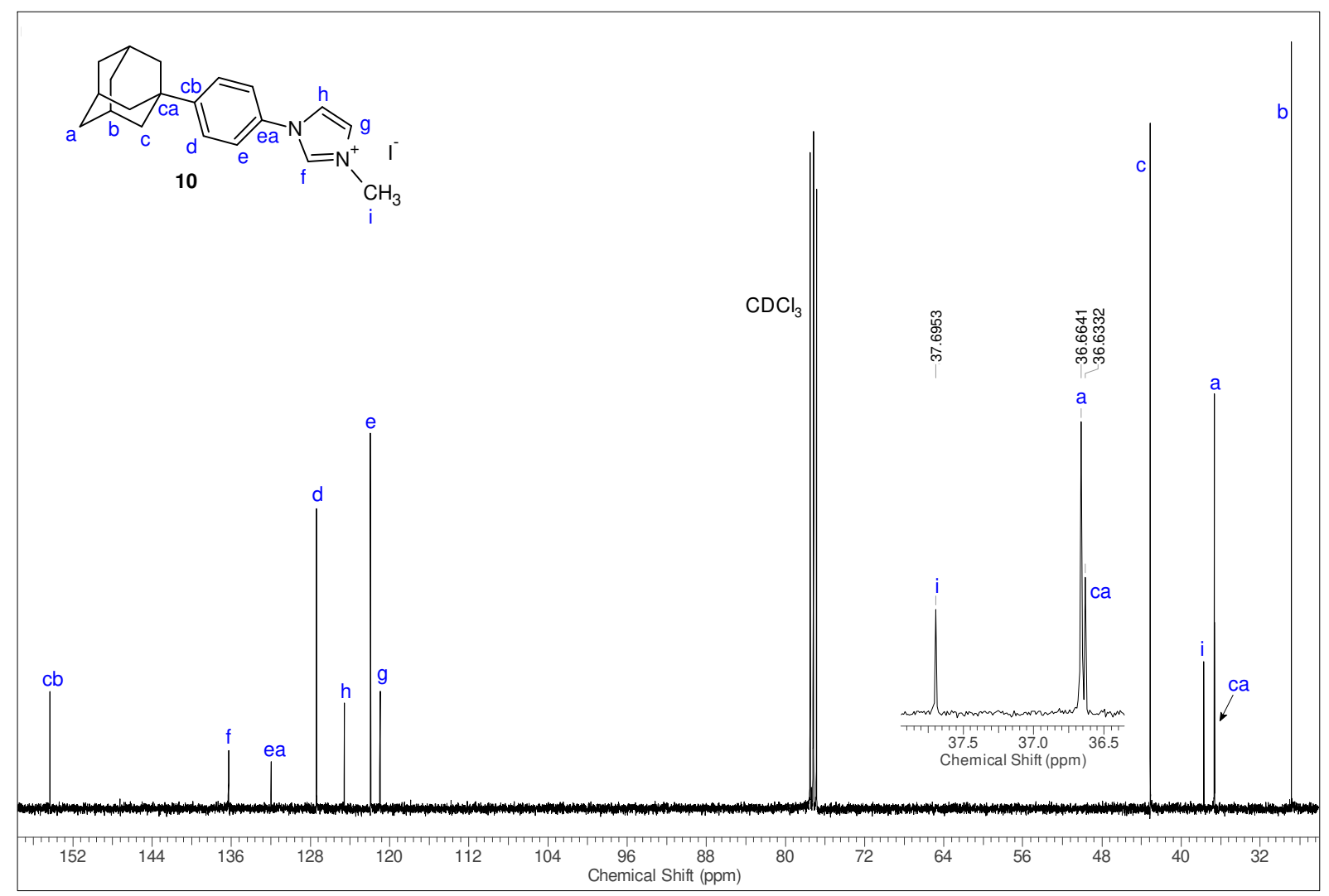

Figure S14 The ${ }^{13} \mathrm{C}\left\{{ }^{1} \mathrm{H}\right\}$ NMR $\left(\mathrm{CDCl}_{3}, 126 \mathrm{MHz}\right)$ spectrum of compound 10. 


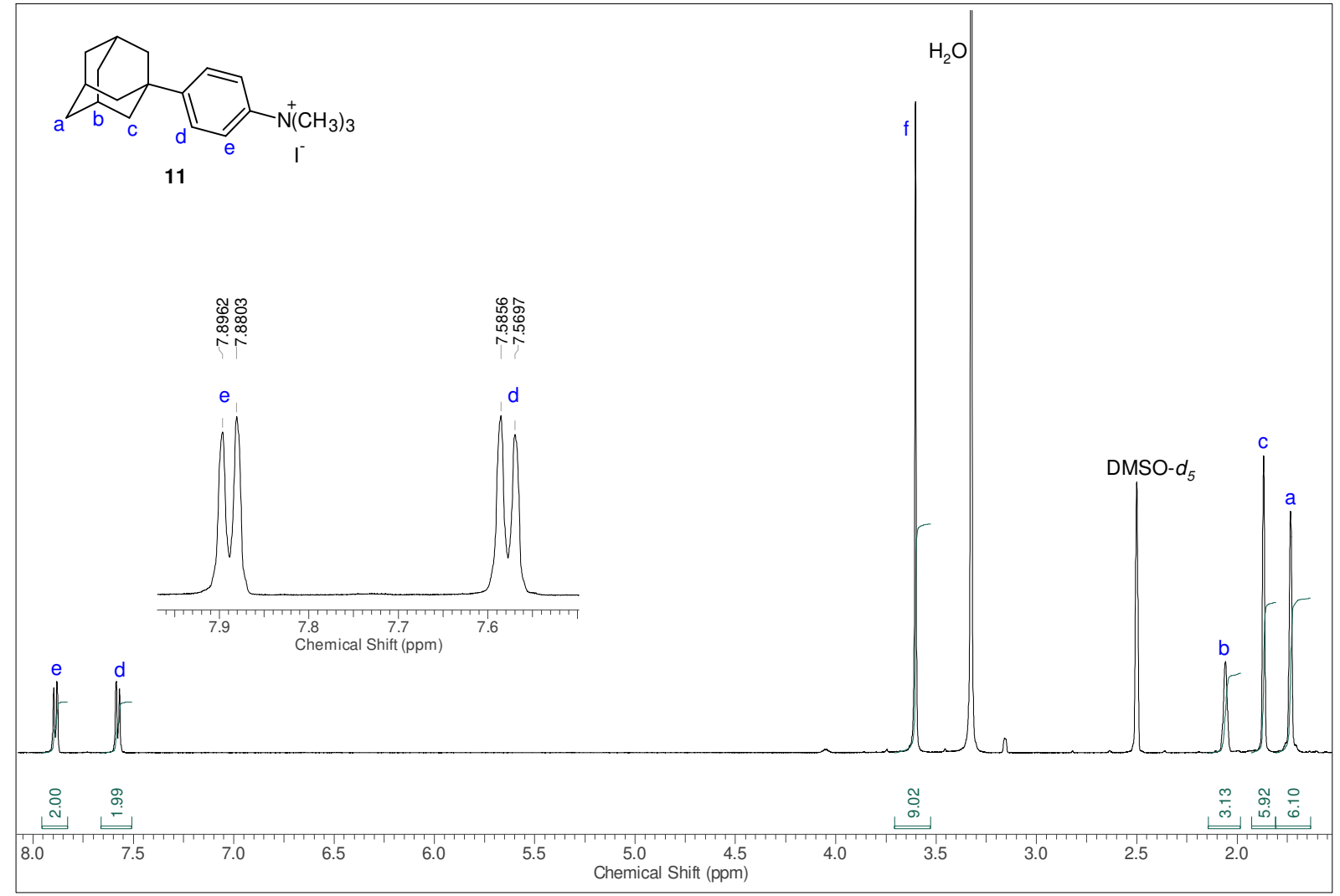

Figure S15 The ${ }^{1} \mathrm{H}$ NMR (DMSO- $d_{6}, 500 \mathrm{MHz}$ ) spectrum of compound 11.

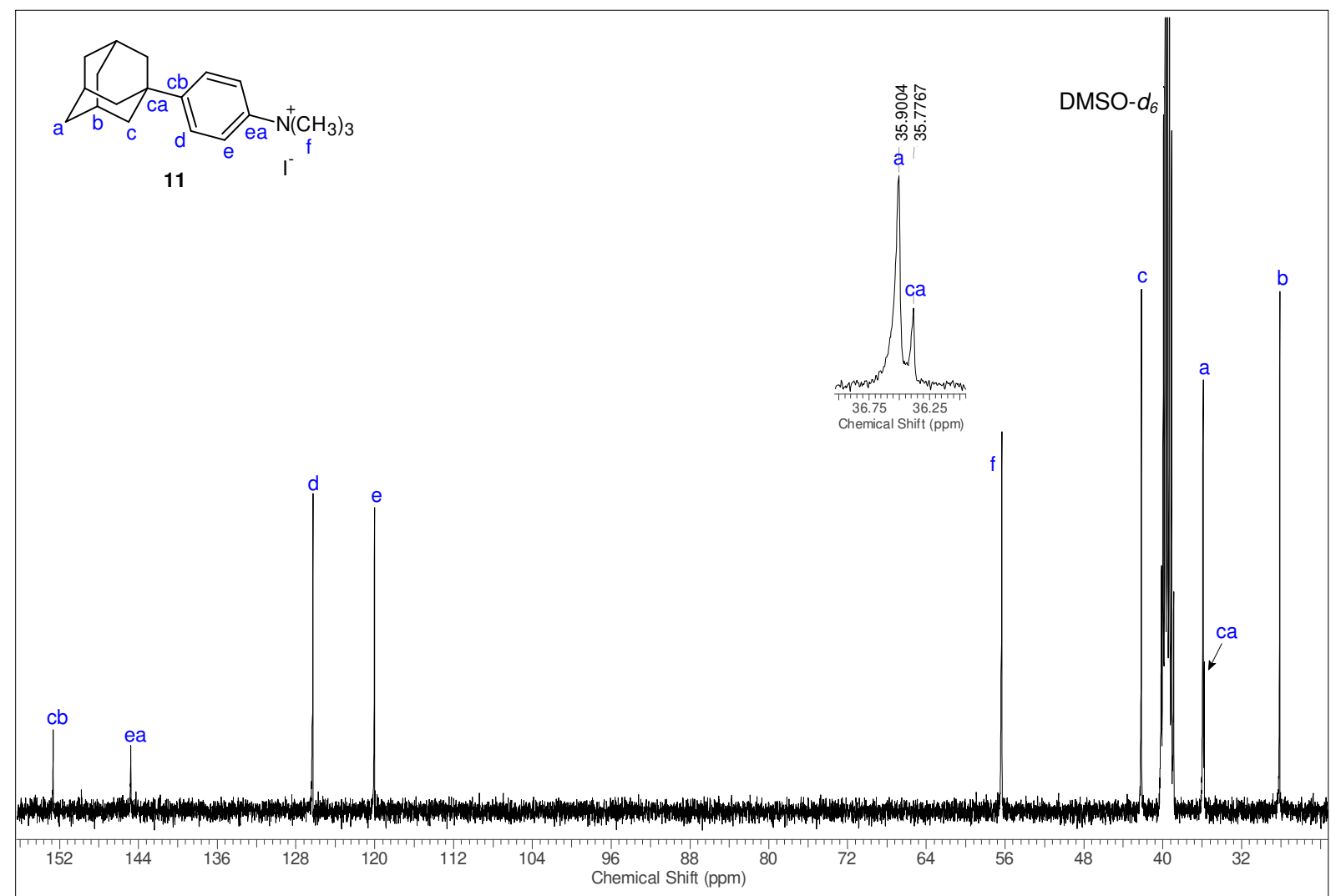

Figure S16 The ${ }^{13} \mathrm{C}\left\{{ }^{1} \mathrm{H}\right\}$ NMR (DMSO- $d_{6}, 126 \mathrm{MHz}$ ) spectrum of compound 11. 


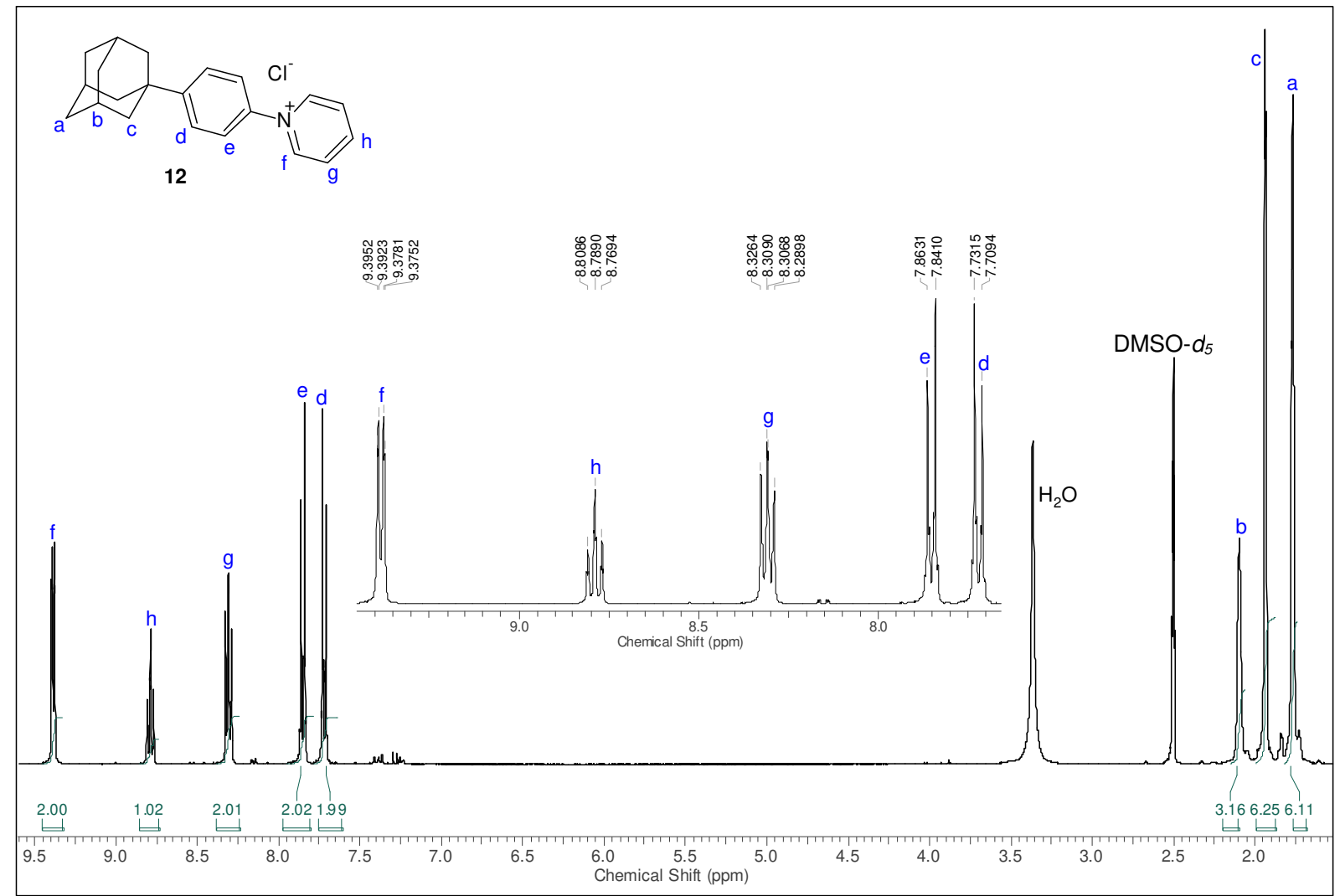

Figure S17 The ${ }^{1} \mathrm{H}$ NMR (DMSO- $d_{6}, 400 \mathrm{MHz}$ ) spectrum of compound 12.

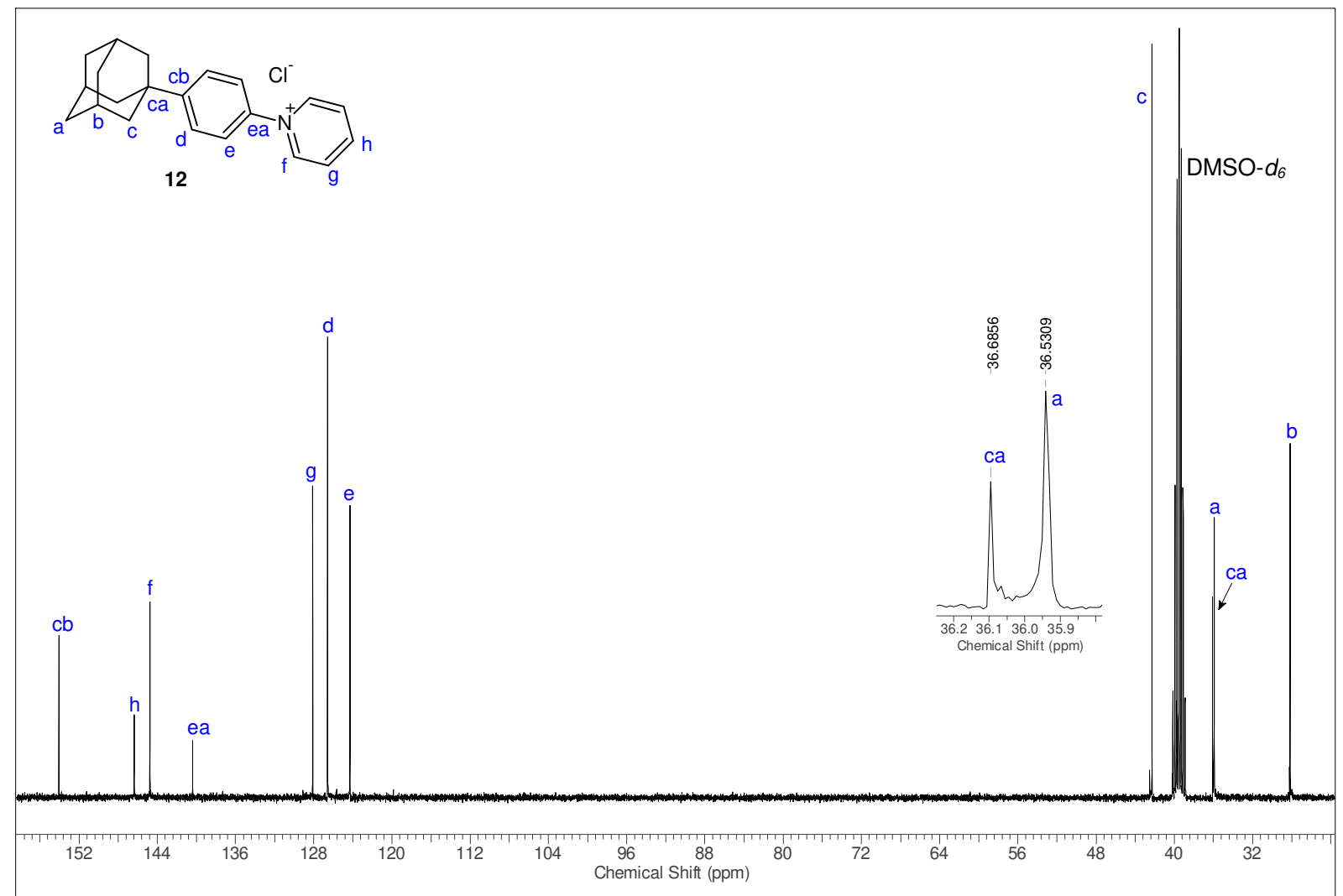

Figure S18 The ${ }^{13} \mathrm{C}\left\{{ }^{1} \mathrm{H}\right\}$ NMR (DMSO- $d_{6}, 101 \mathrm{MHz}$ ) spectrum of compound 12. 
Stacking plots of NMR titrations.

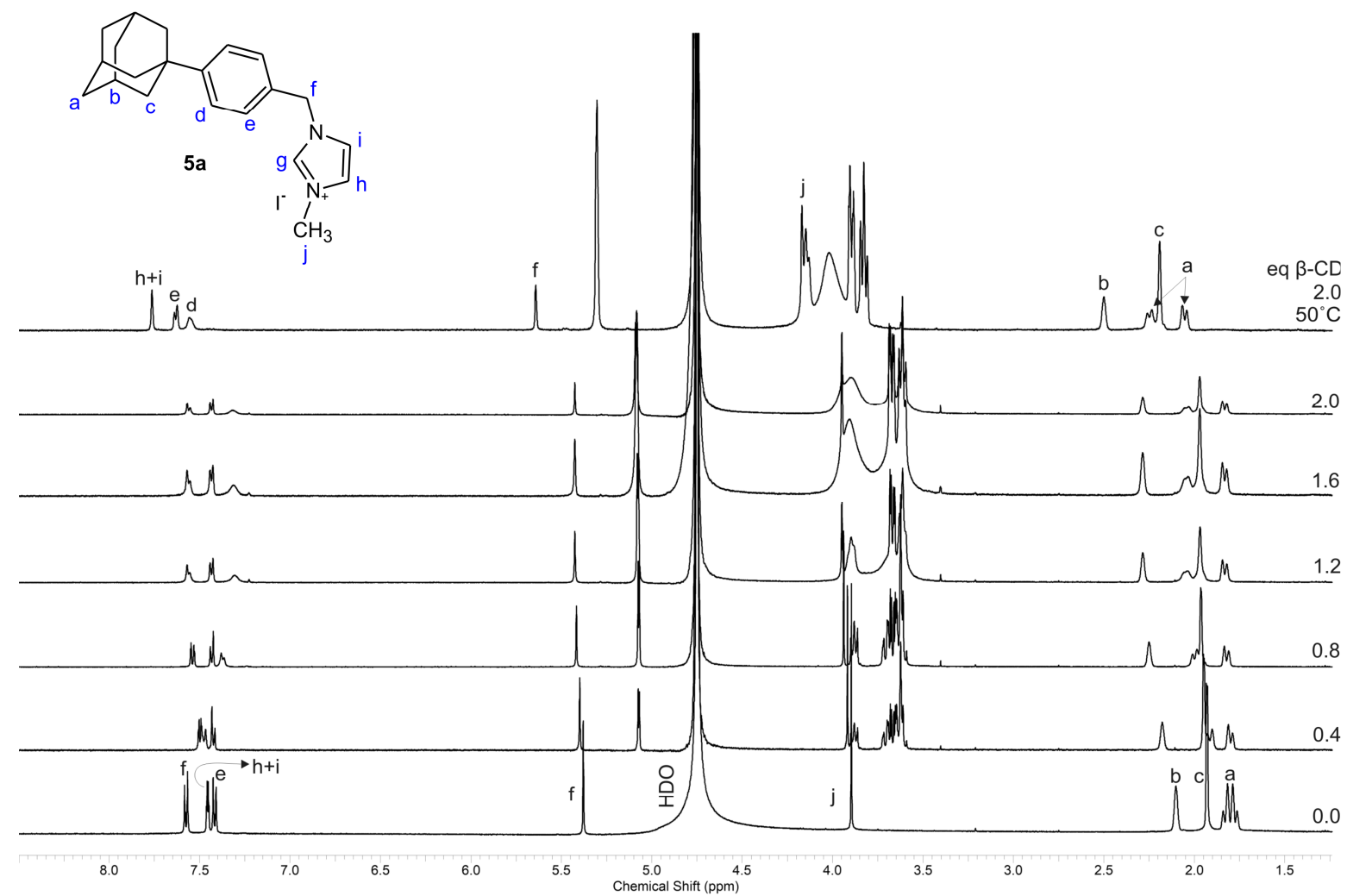

Figure S19 Stacking plot of ${ }^{1} \mathrm{H}$ NMR spectra $(400 \mathrm{MHz})$ of mixtures of $\mathbf{5 a}$ with $\beta-\mathrm{CD}\left(\mathrm{D}_{2} \mathrm{O}, 303 \mathrm{~K}\right.$ if not stated otherwise).

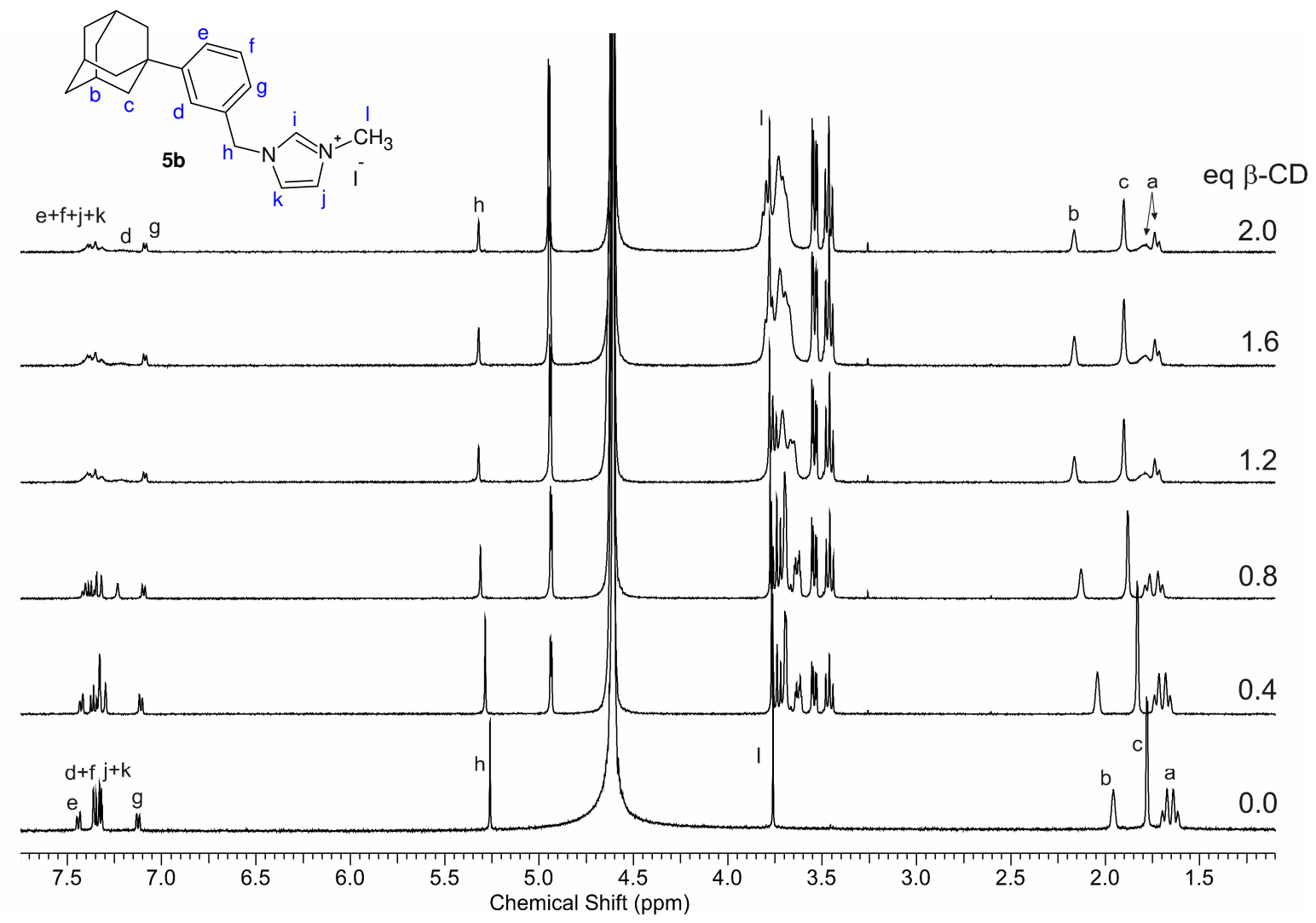

Figure S20 Stacking plot of ${ }^{1} \mathrm{H}$ NMR spectra $(400 \mathrm{MHz})$ of mixtures of $\mathbf{5 b}$ with $\beta-\mathrm{CD}\left(\mathrm{D}_{2} \mathrm{O}, 303 \mathbf{K}\right)$. 


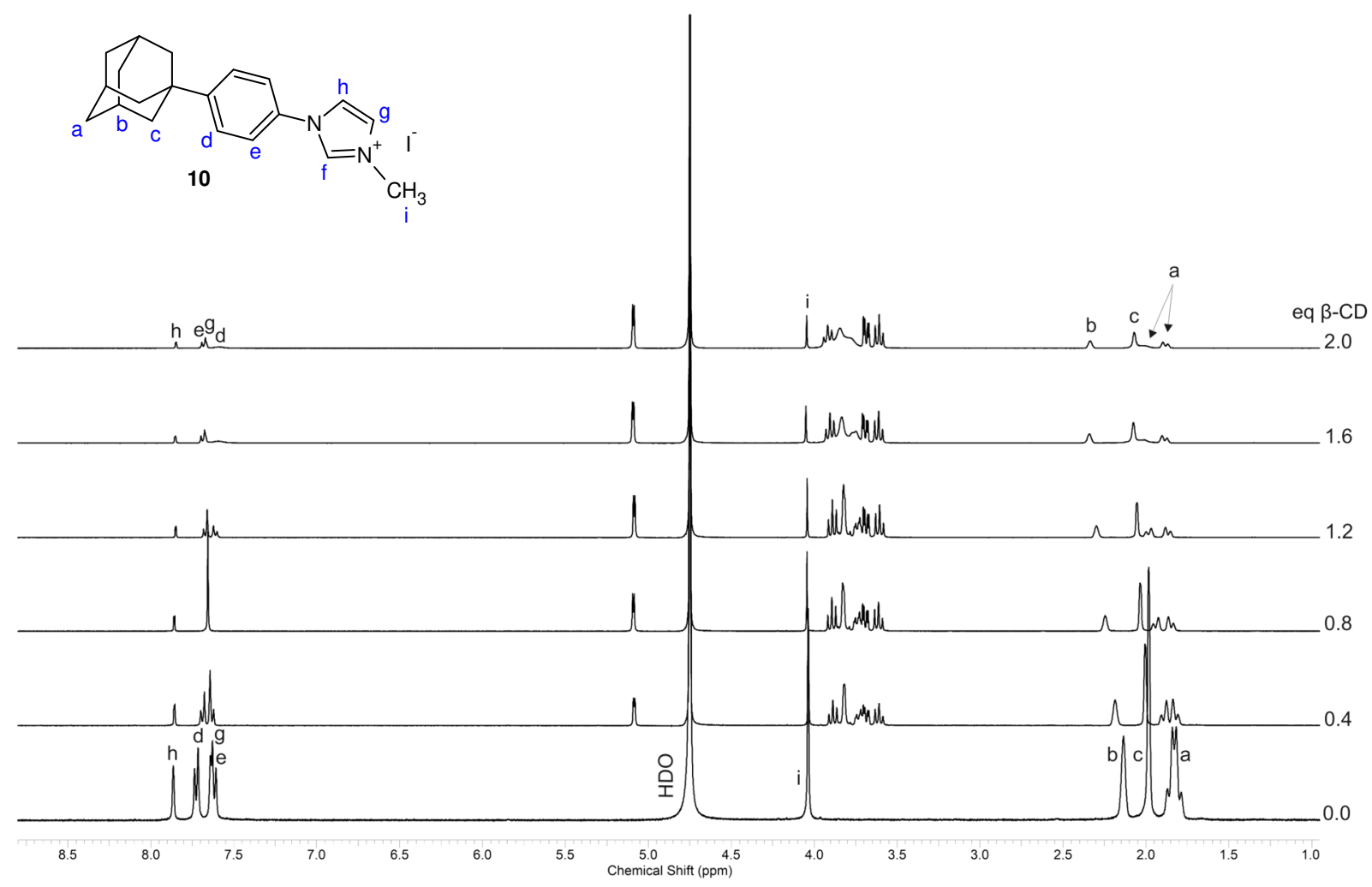

Figure S21 Stacking plot of ${ }^{1} \mathrm{H}$ NMR spectra $(400 \mathrm{MHz})$ of mixtures of $\mathbf{1 0}$ with $\beta$-CD $\left(\mathrm{D}_{2} \mathrm{O}, 303 \mathrm{~K}\right)$.

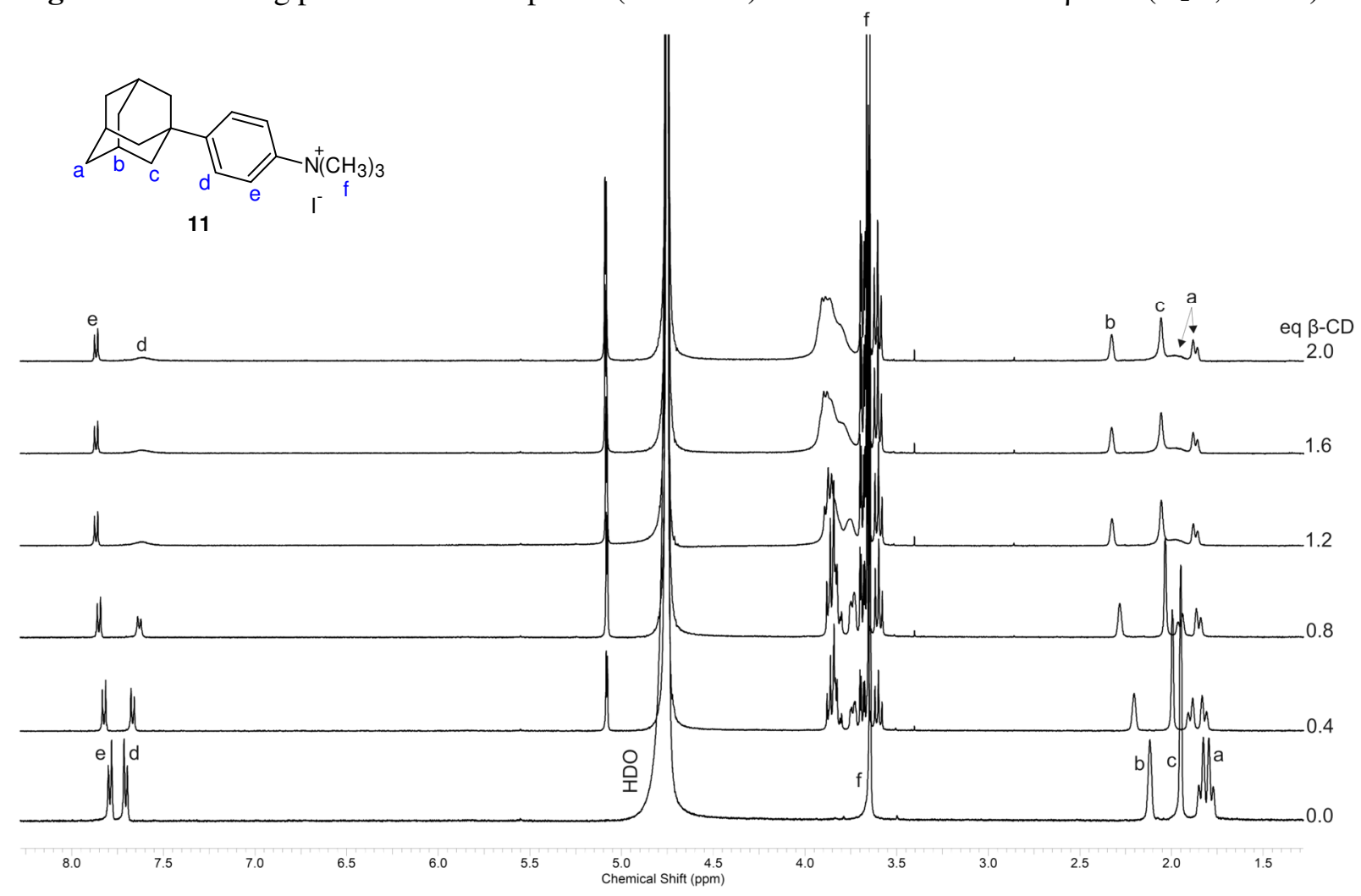

Figure S22 Stacking plot of ${ }^{1} \mathrm{H}$ NMR spectra $(400 \mathrm{MHz})$ of mixtures of $\mathbf{1 1}$ with $\beta$-CD $\left(\mathrm{D}_{2} \mathrm{O}, 303 \mathrm{~K}\right)$. 

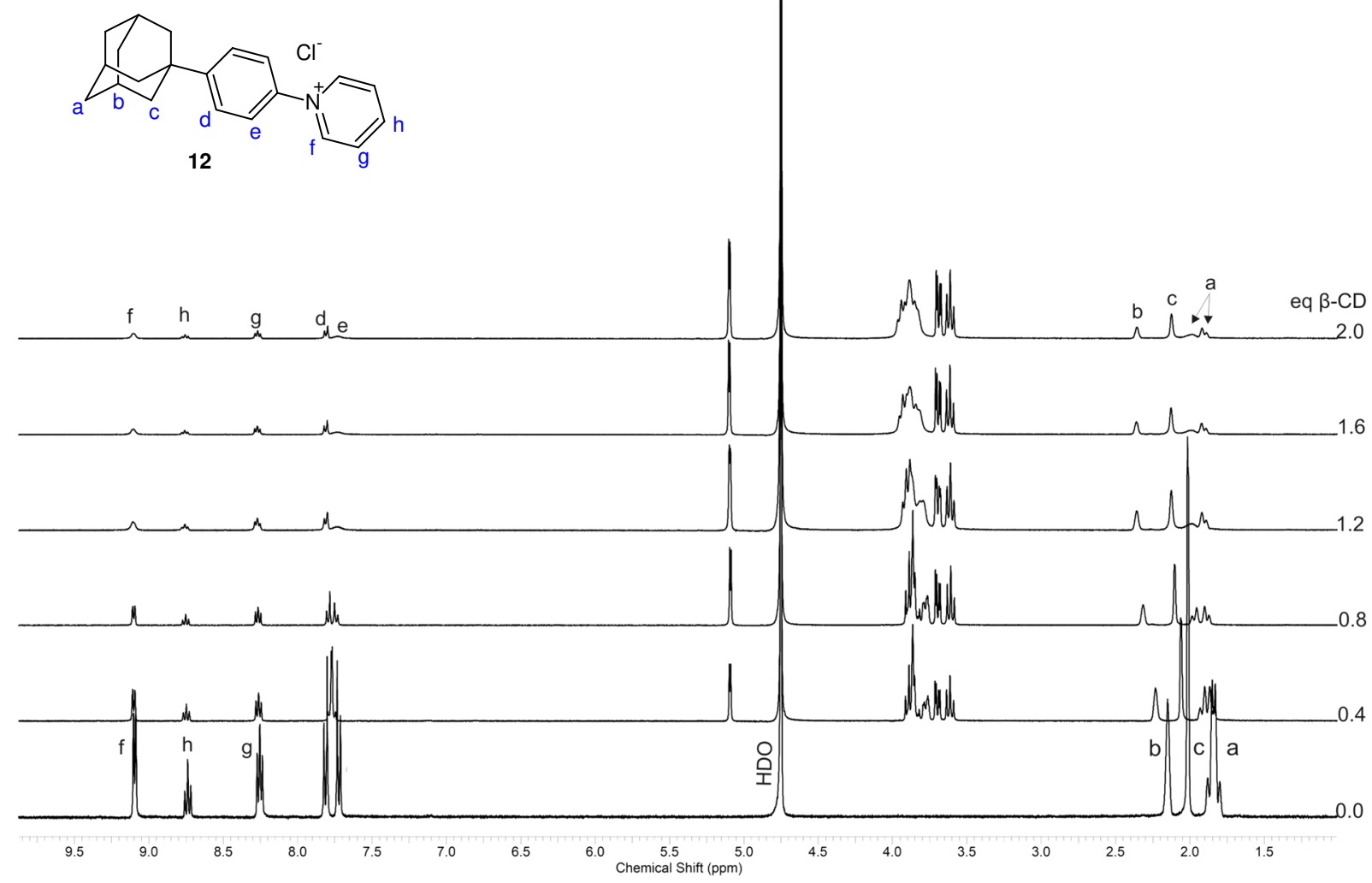

Figure S23 Stacking plot of ${ }^{1} \mathrm{H}$ NMR spectra (400 MHz) of mixtures of 12 with $\beta-\mathrm{CD}\left(\mathrm{D}_{2} \mathrm{O}, 303 \mathrm{~K}\right)$.

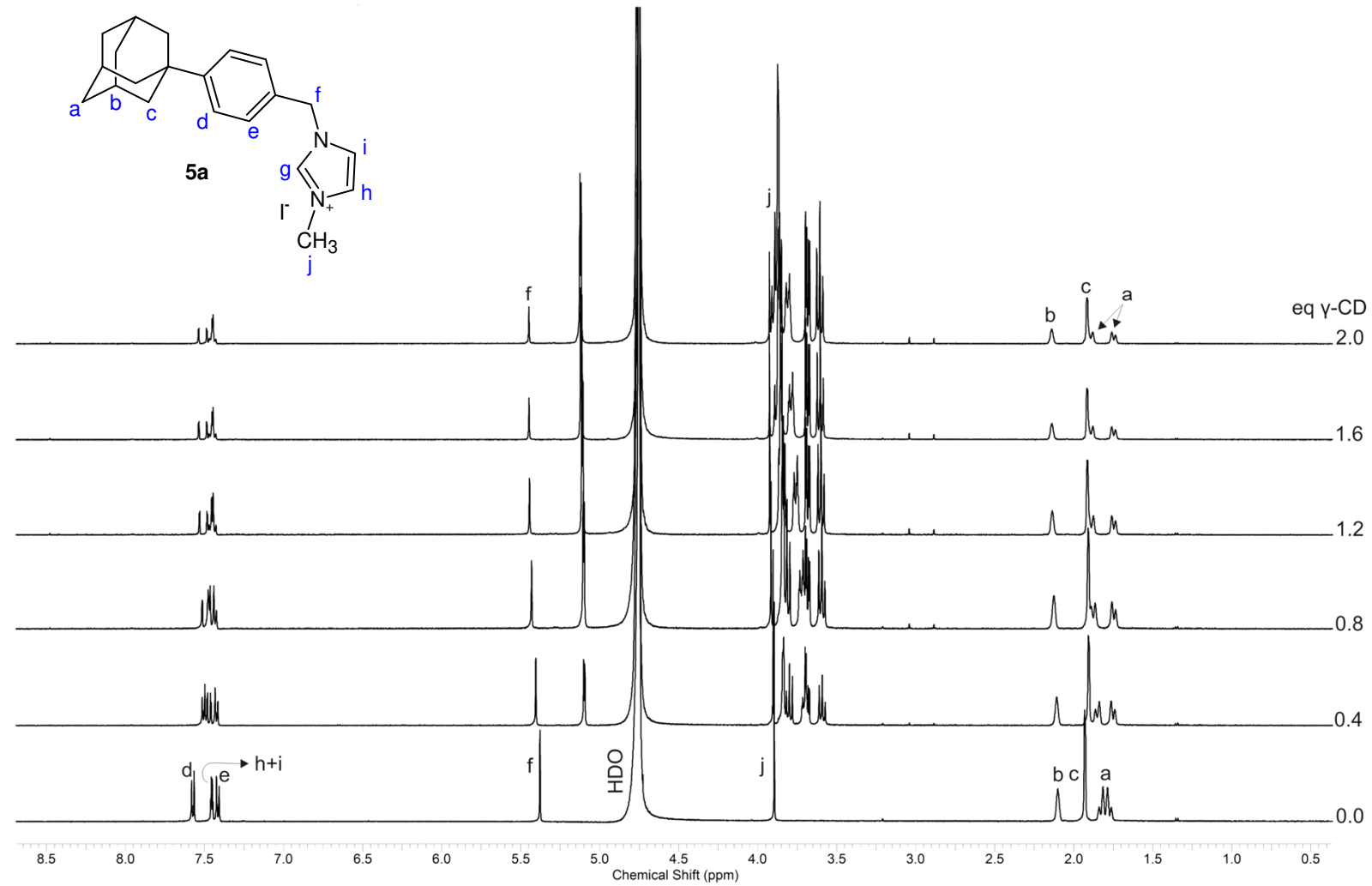

Figure S24 Stacking plot of ${ }^{1} \mathrm{H}$ NMR spectra $(400 \mathrm{MHz})$ of mixtures of $\mathbf{5 a}$ with $\gamma$-CD $\left(\mathrm{D}_{2} \mathrm{O}, 303 \mathrm{~K}\right)$. 


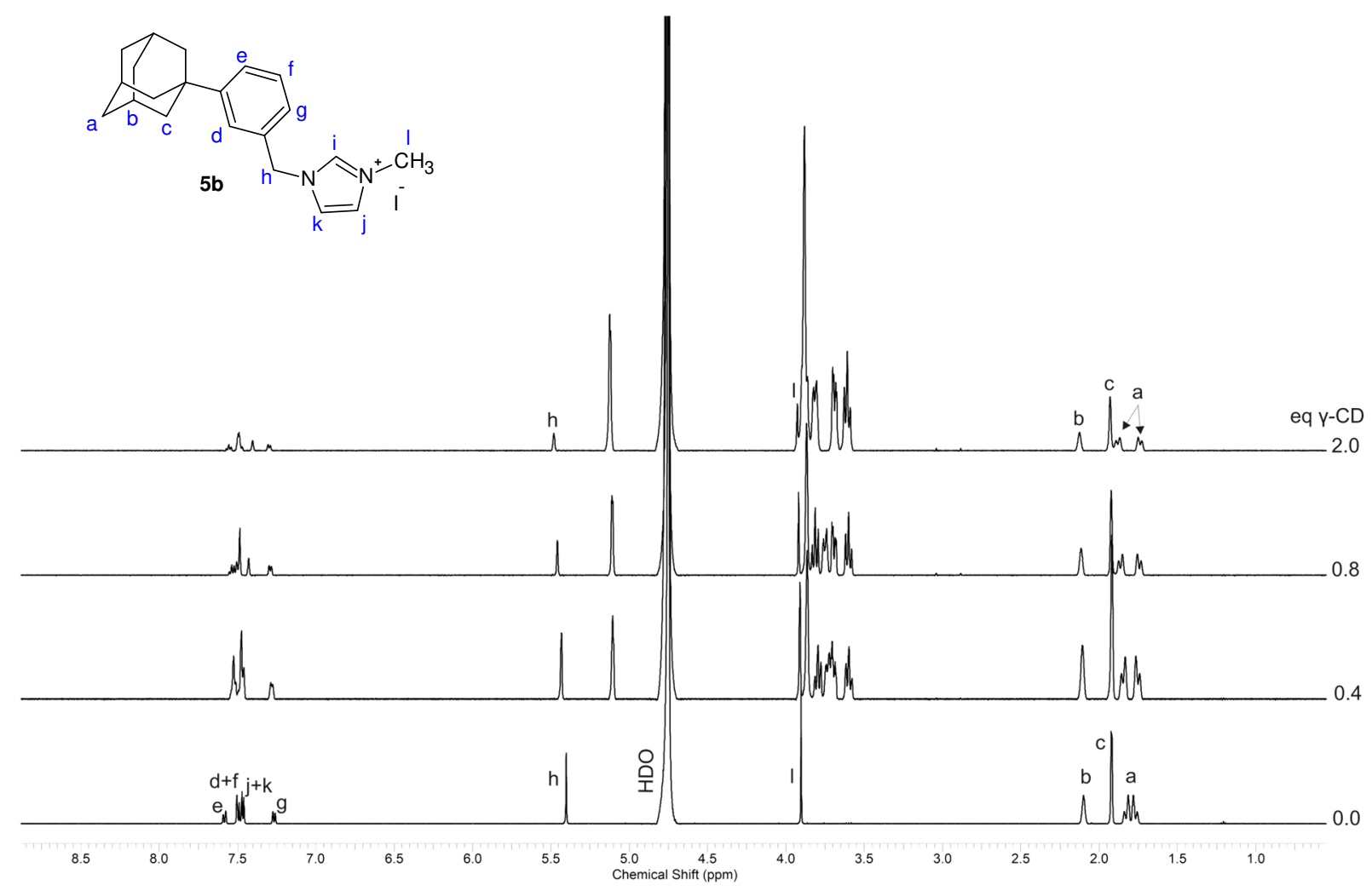

Figure S25 Stacking plot of ${ }^{1} \mathrm{H}$ NMR spectra (400 MHz) of mixtures of $\mathbf{5 b}$ with $\gamma$-CD $\left(\mathrm{D}_{2} \mathrm{O}, 303 \mathbf{K}\right)$.

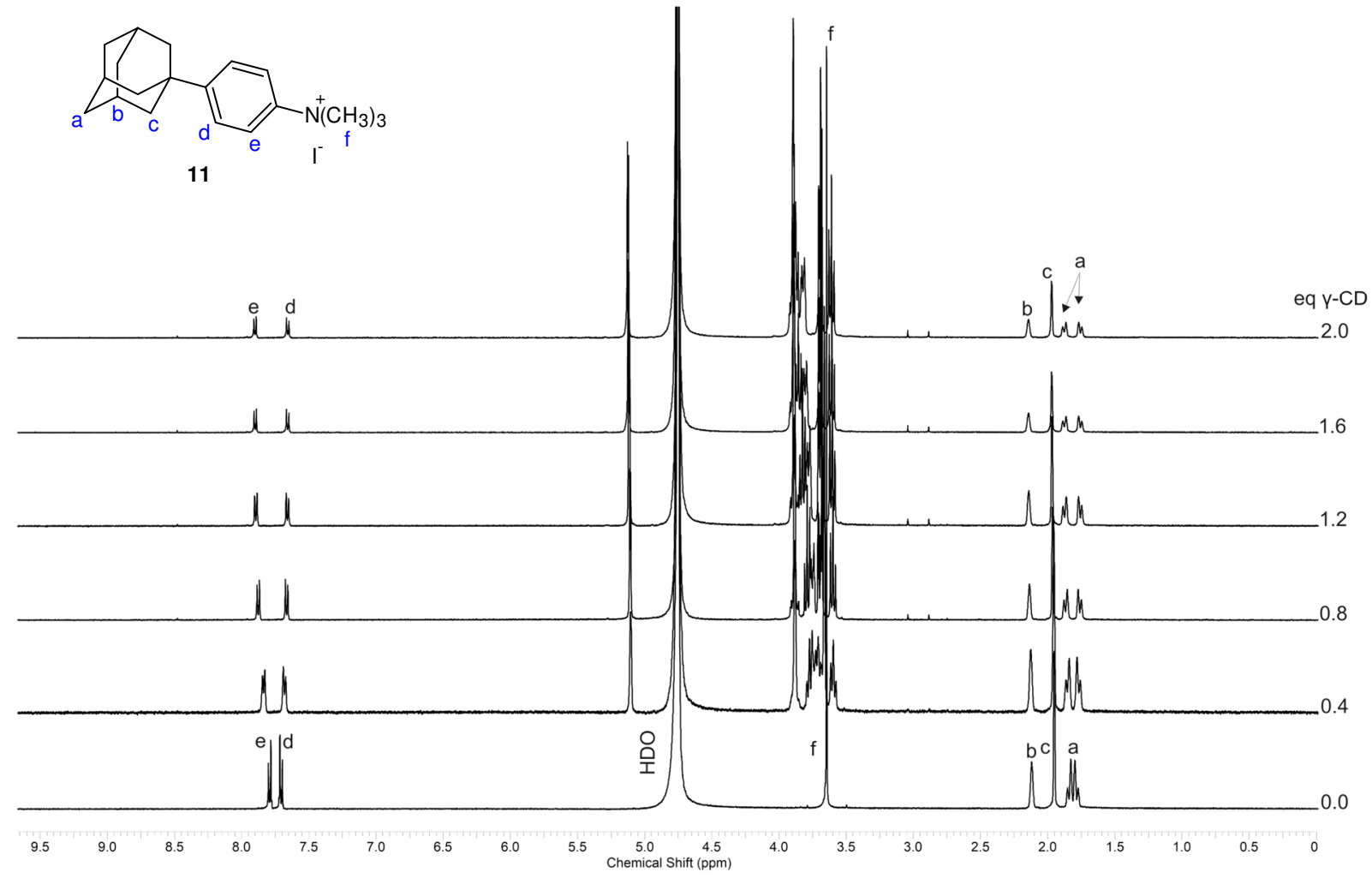

Figure S26 Stacking plot of ${ }^{1} \mathrm{H}$ NMR spectra (400 MHz) of mixtures of 11 with $\gamma-\mathrm{CD}\left(\mathrm{D}_{2} \mathrm{O}, 303 \mathrm{~K}\right)$. 


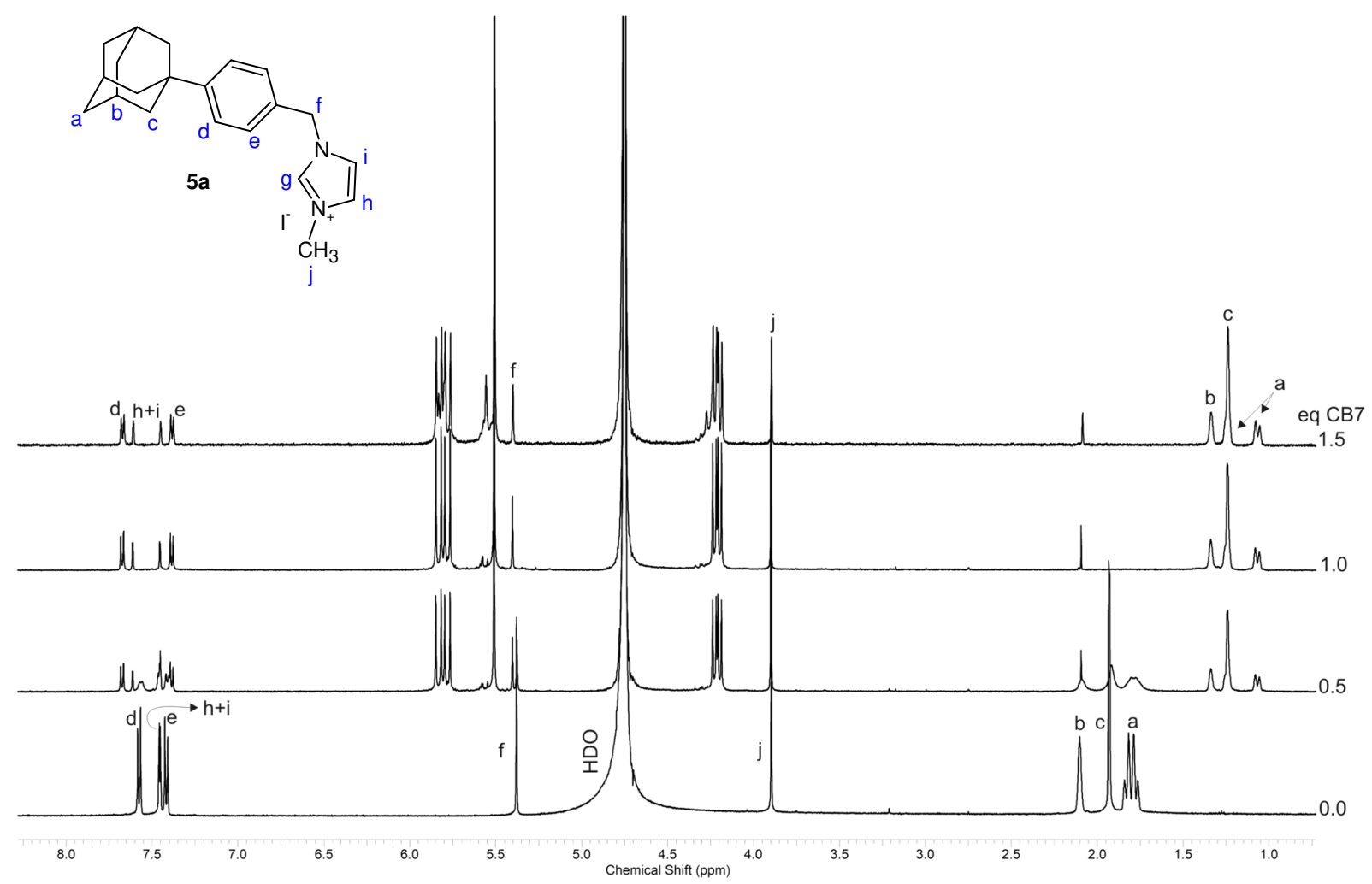

Figure S27 Stacking plot of ${ }^{1} \mathrm{H}$ NMR spectra (400 MHz) of mixtures of 5a with CB7 ( $\left.\mathrm{D}_{2} \mathrm{O}, 303 \mathrm{~K}\right)$.

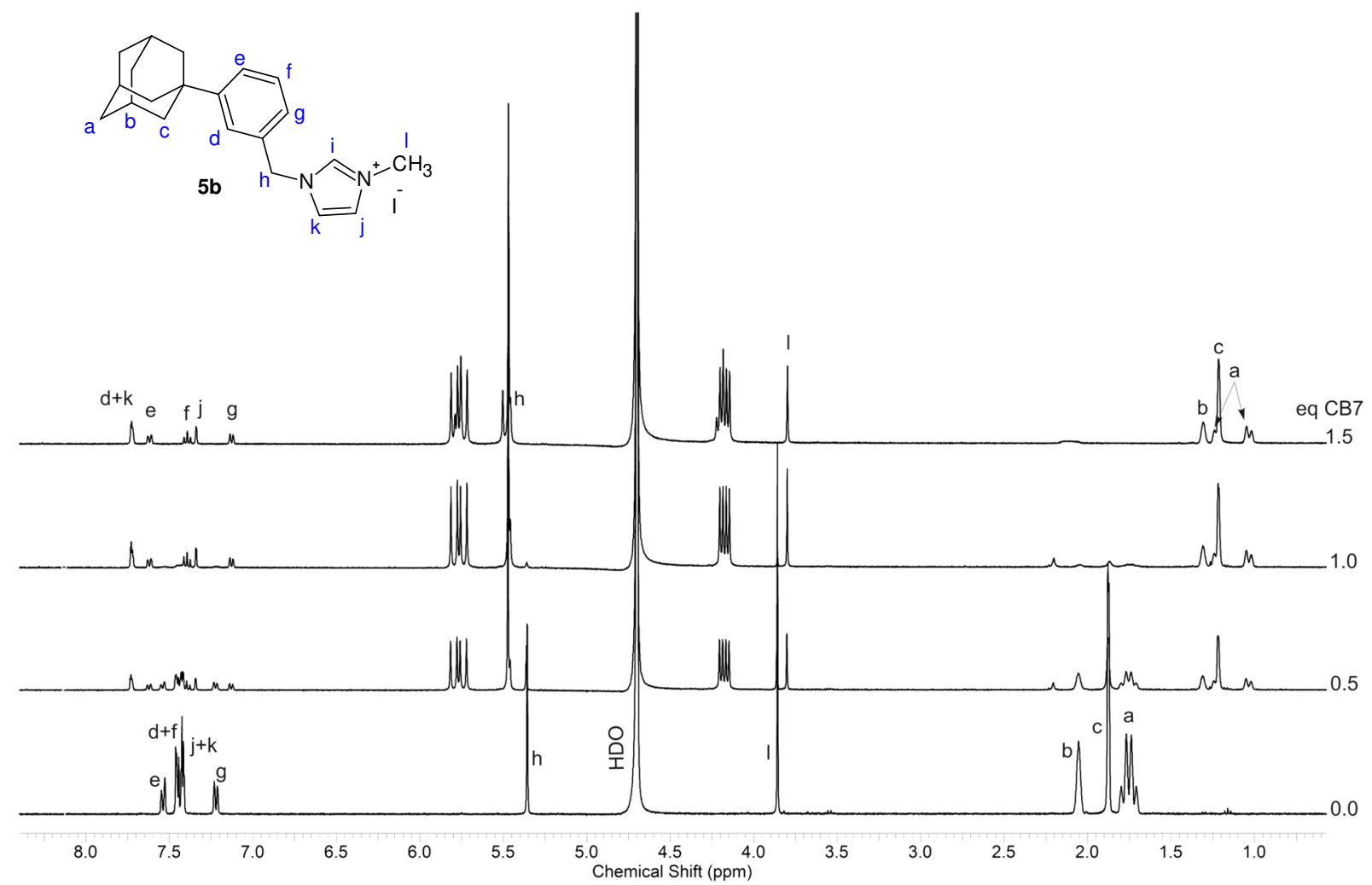

Figure S28 Stacking plot of ${ }^{1} \mathrm{H}$ NMR spectra (400 MHz) of mixtures of $\mathbf{5 b}$ with CB7 ( $\left.\mathrm{D}_{2} \mathrm{O}, 303 \mathbf{K}\right)$. 


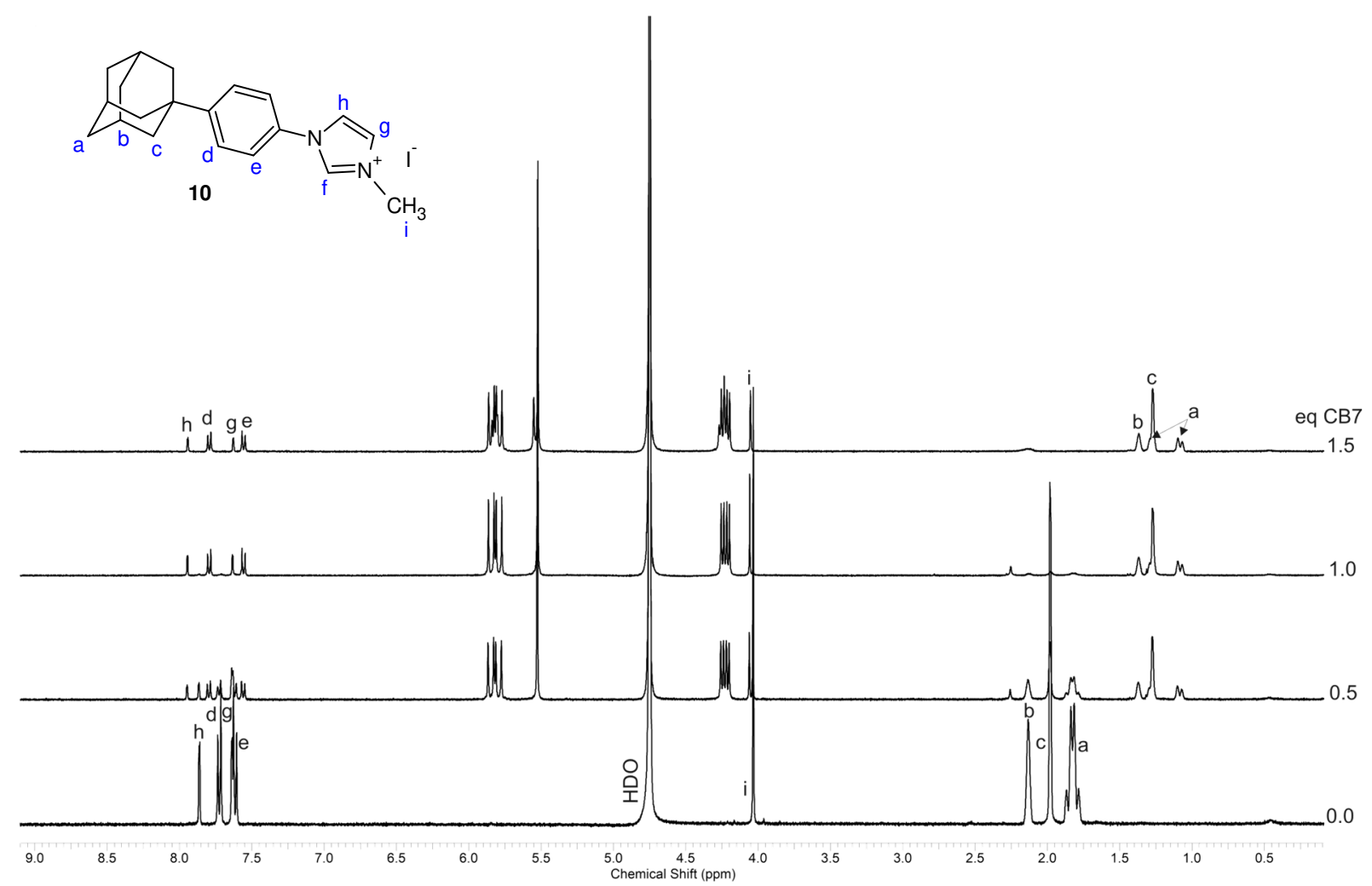

Figure S29 Stacking plot of ${ }^{1} \mathrm{H}$ NMR spectra $(400 \mathrm{MHz})$ of mixtures of $\mathbf{1 0}$ with $\mathrm{CB} 7\left(\mathrm{D}_{2} \mathrm{O}, 303 \mathrm{~K}\right)$.

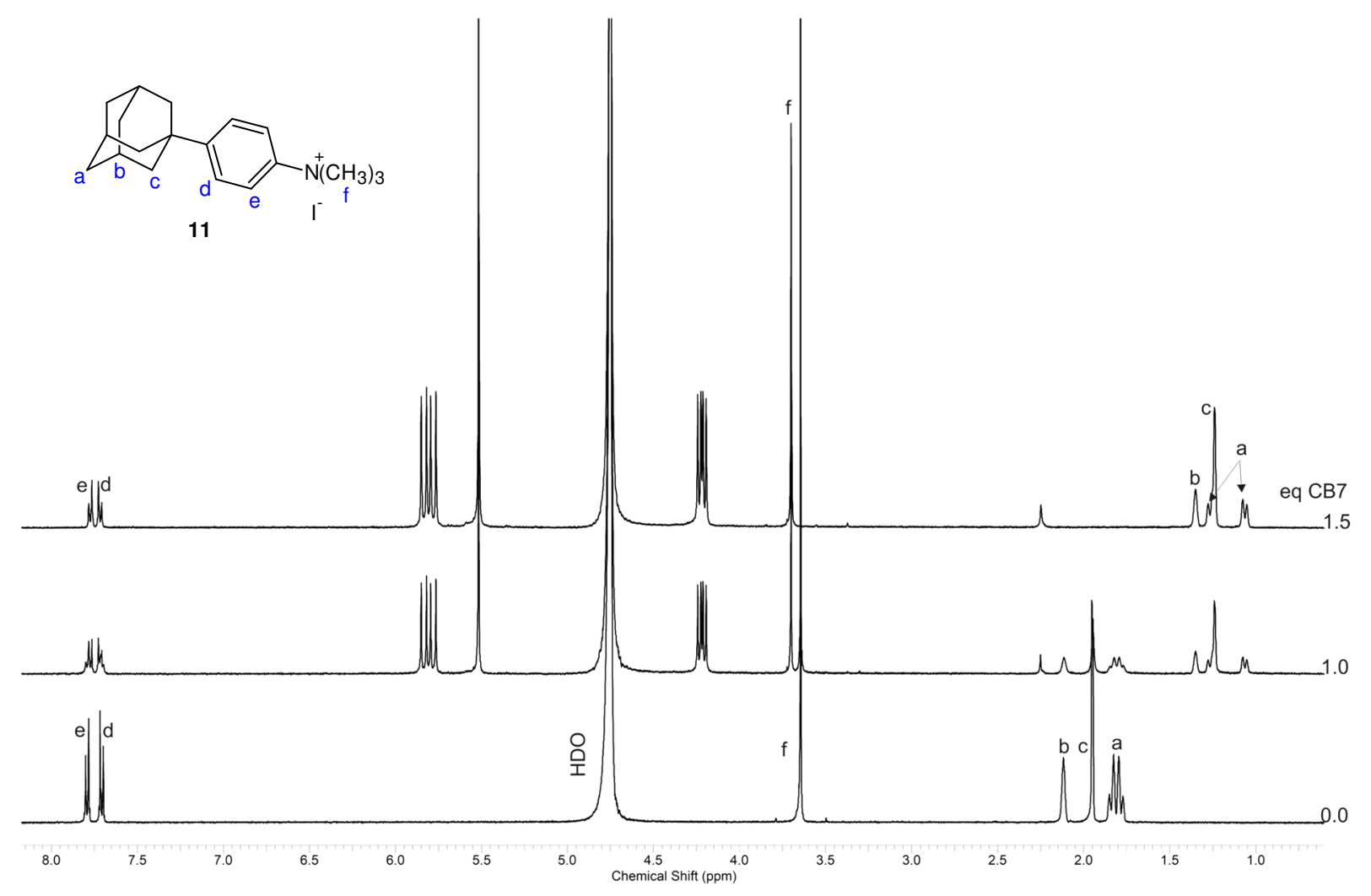

Figure S30 Stacking plot of ${ }^{1} \mathrm{H}$ NMR spectra (400 MHz) of mixtures of $\mathbf{1 1}$ with $\mathrm{CB} 7\left(\mathrm{D}_{2} \mathrm{O}, 303 \mathrm{~K}\right)$. 


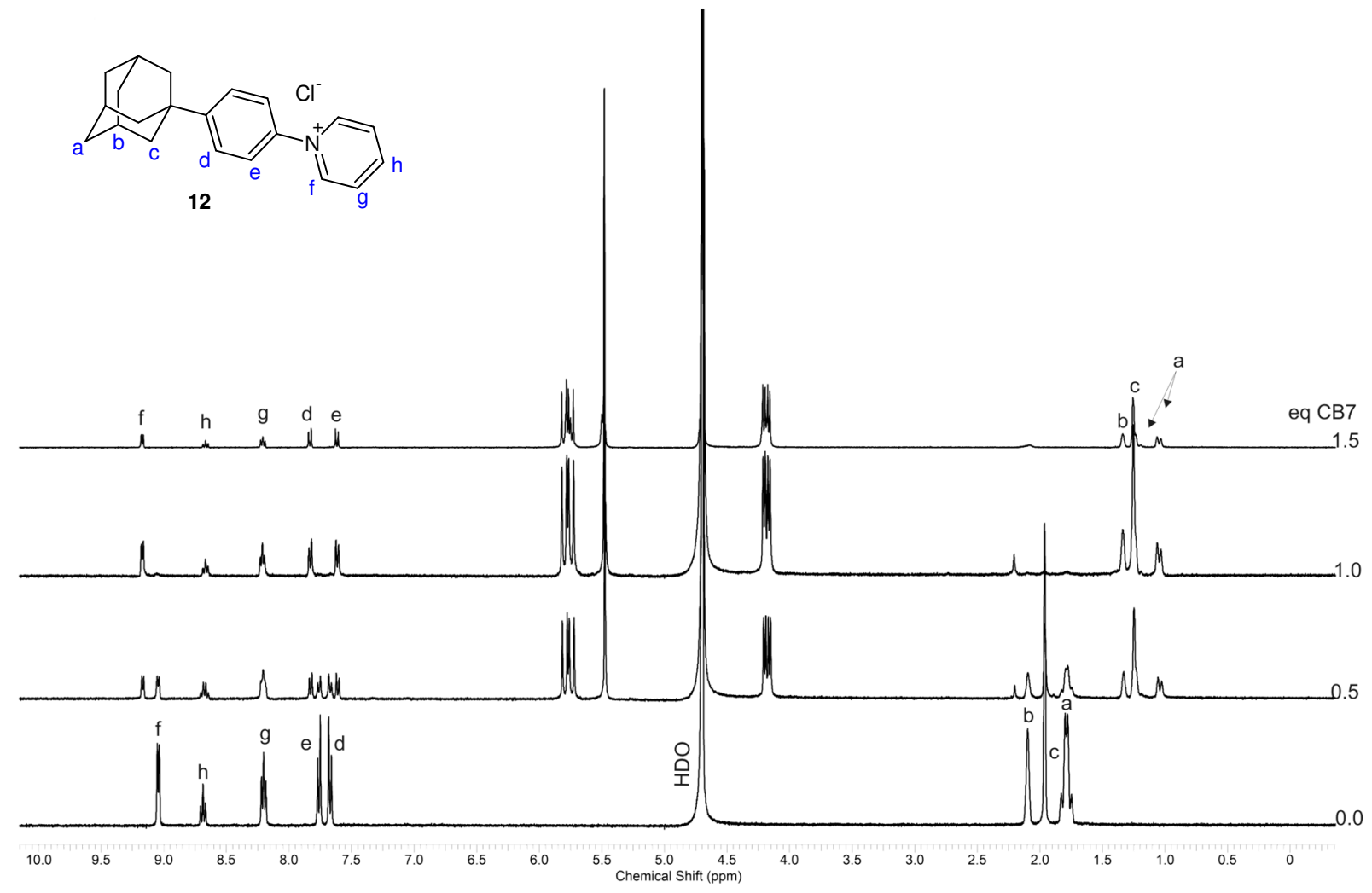

Figure S31 Stacking plot of ${ }^{1} \mathrm{H}$ NMR spectra (400 MHz) of mixtures of 12 with $\mathrm{CB} 7\left(\mathrm{D}_{2} \mathrm{O}, 303 \mathrm{~K}\right)$.

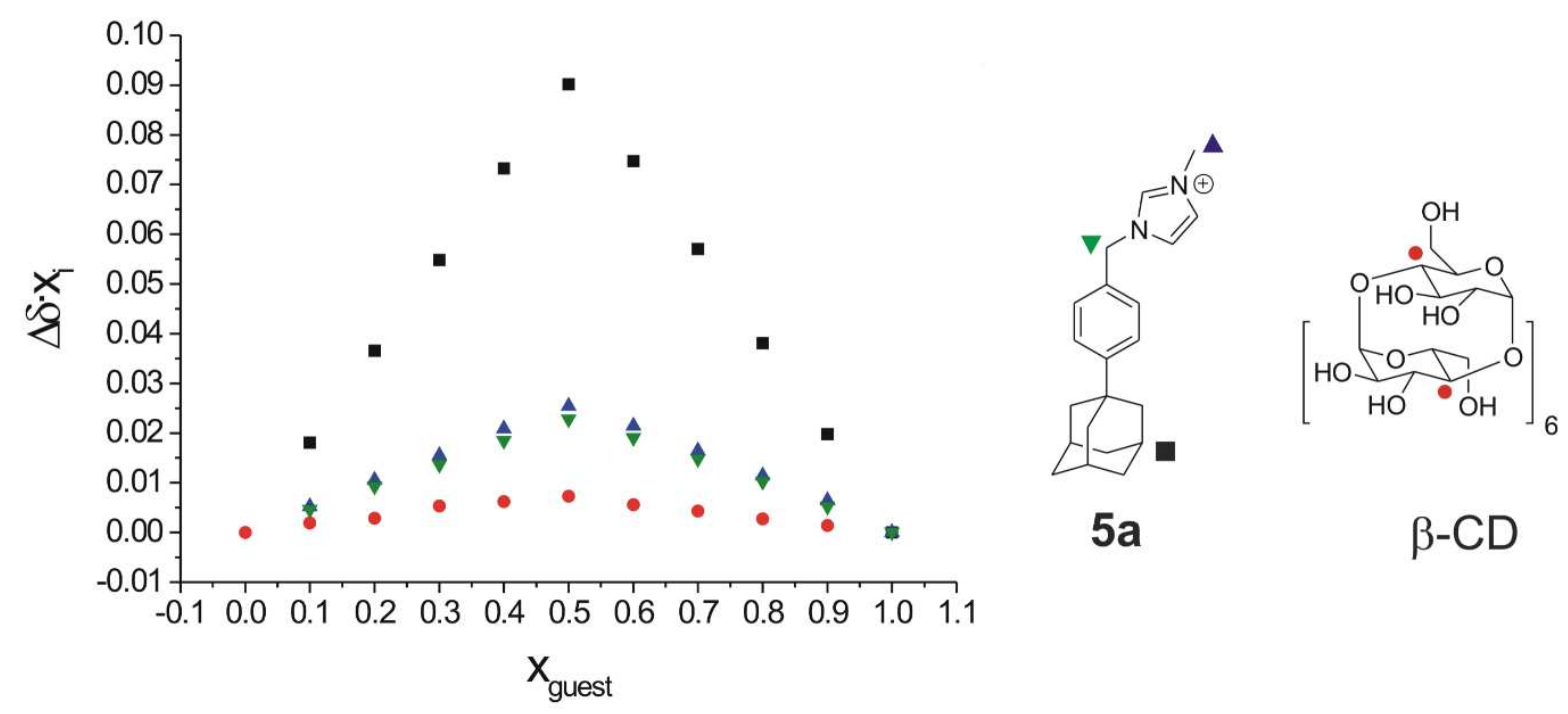

Figure S32 Job's plot for the guest $5 \mathbf{a}\left(500 \mathrm{MHz}, 303 \mathrm{~K}, \mathrm{D}_{2} \mathrm{O}\right)$. For $y$-axis: $x_{\mathrm{i}}=$ molar fraction of guest or host. 


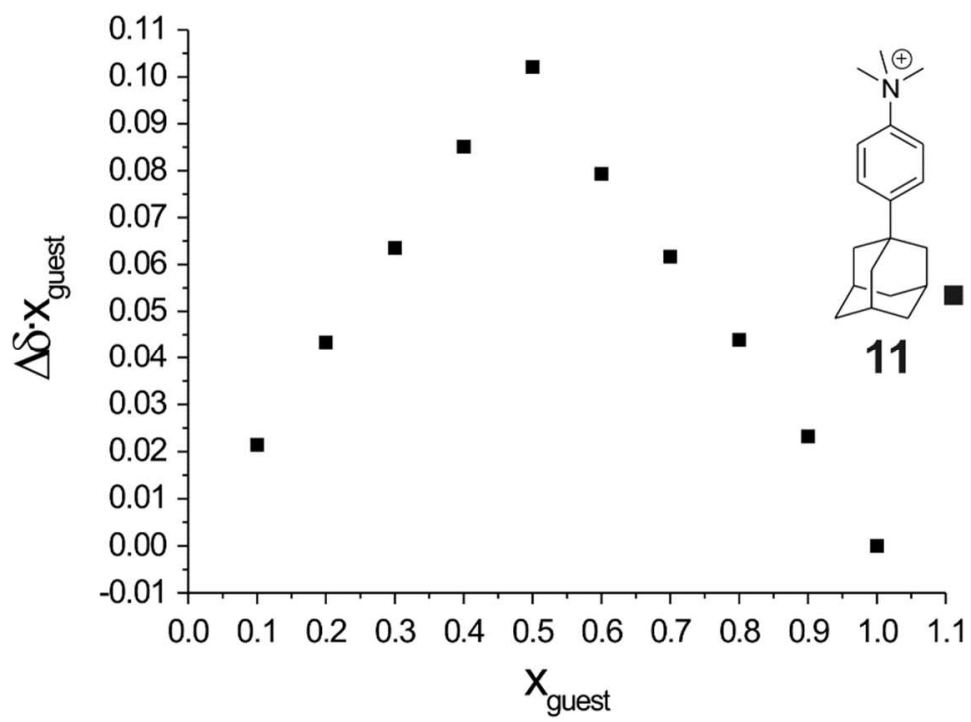

Figure S33 Job's plot for the guest $11\left(500 \mathrm{MHz}, 303 \mathrm{~K}, \mathrm{D}_{2} \mathrm{O}\right)$. 
ITC data for the guests $5 \mathrm{a}, \mathbf{5 b}, \mathbf{1 0 - 1 2}$

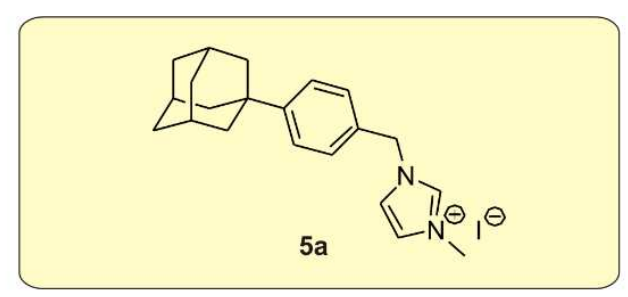

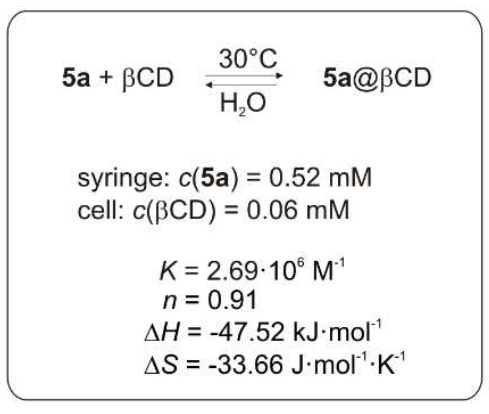

Time (min)

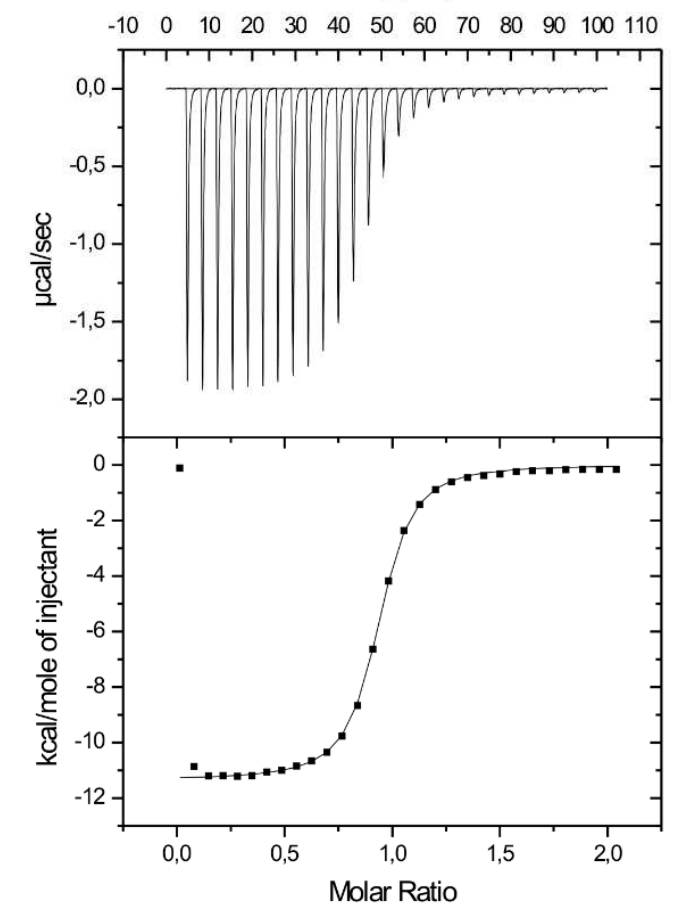

$5 a+C B 7 \underset{\mathrm{H}_{2} \mathrm{O}}{\stackrel{30^{\circ} \mathrm{C}}{\longrightarrow}} 5 \mathrm{a} @ \mathrm{CB} 7$

syringe: $c(5 \mathbf{a})=0.45 \mathrm{mM}$ cell: $c(\mathrm{CB} 7)=0.05 \mathrm{mM}$ $c($ HeMelmCl $)=0.41 \mathrm{mM}$

$K=6.61 \cdot 10^{9} \mathrm{M}^{-1}$ $n=1.06$

$\Delta H=-81.01 \mathrm{~kJ} \cdot \mathrm{mol}^{-1}$ $\Delta S=-79.12 \mathrm{~J} \cdot \mathrm{mol}^{-1} \cdot \mathrm{K}^{-1}$

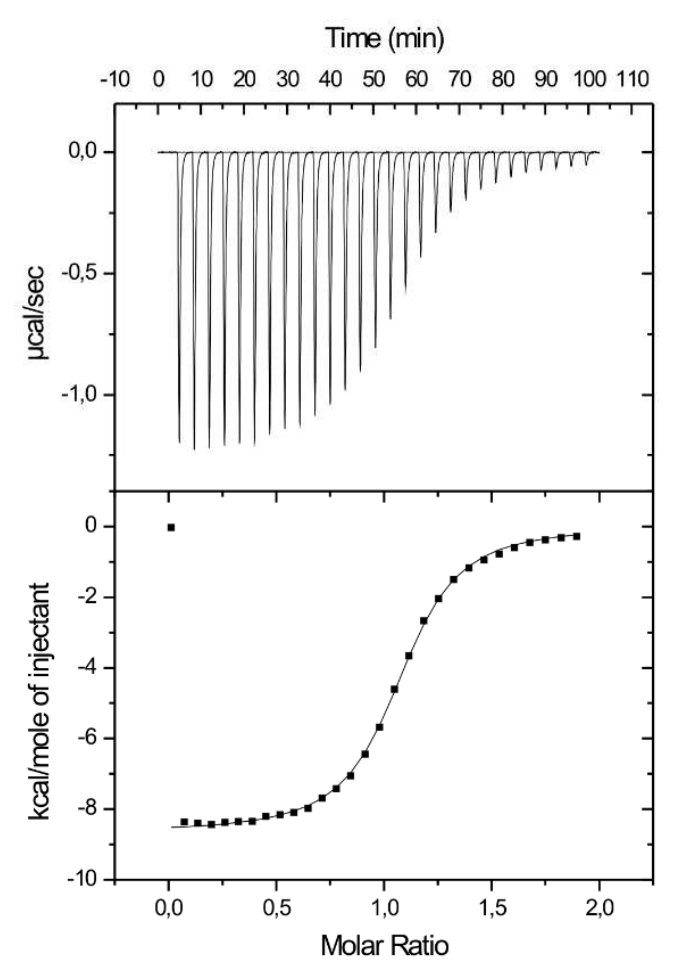

Figure S34 ITC data for the guest 5a and $\beta-C D$ and CB7 


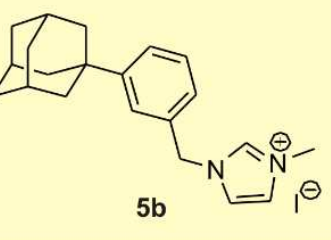

$5 \mathbf{b}+\beta C D \underset{\mathrm{H}_{2} \mathrm{O}}{\stackrel{30^{\circ} \mathrm{C}}{\longrightarrow}} 5 \mathbf{b} @ \beta C D$

syringe: $c(\mathbf{5 b})=0.53 \mathrm{mM}$ cell: $c(\beta C D)=0.05 \mathrm{mM}$

$K=4.20 \cdot 10^{5} \mathrm{M}^{-1}$ $n=0.98$

$\Delta H=-36.34 \mathrm{~kJ} \cdot \mathrm{mol}^{-1}$ $\Delta S=-12.23 \mathrm{~J} \cdot \mathrm{mol}^{-1} \cdot \mathrm{K}^{-1}$

Time (min)

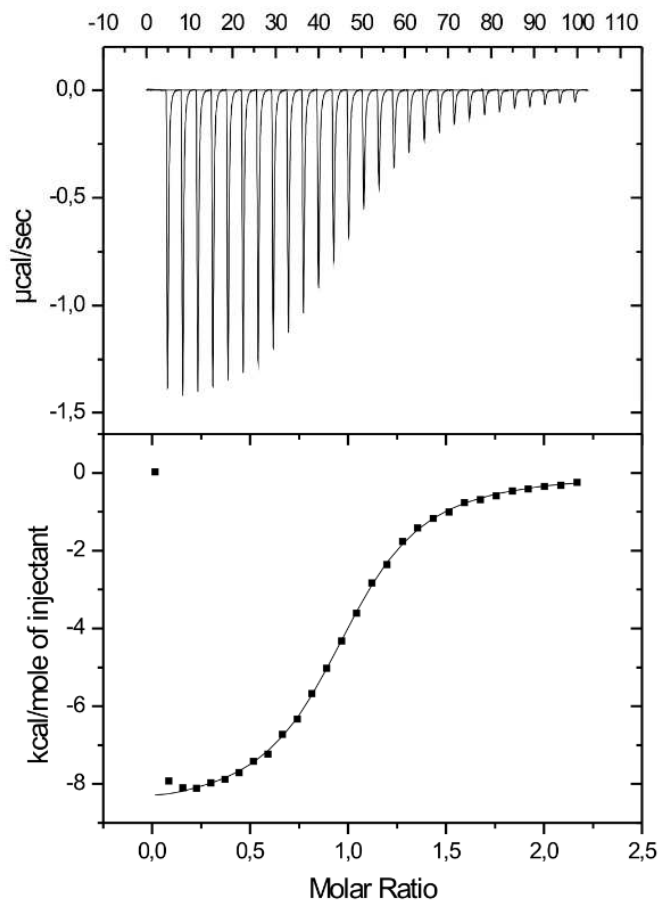

$5 \mathbf{b}+\mathrm{CB} 7 \underset{\mathrm{H}_{2} \mathrm{O}}{\stackrel{30^{\circ} \mathrm{C}}{\longrightarrow}} 5 \mathbf{b} @ \mathrm{CB} 7$

syringe: $c(5 \mathbf{b})=0.49 \mathrm{mM}$ cell: $c(\mathrm{CB} 7)=0.05 \mathrm{mM}$ $c(\mathrm{HeMelmCl})=4.84 \mathrm{mM}$

$K=2.63 \cdot 10^{10} \mathrm{M}^{-1}$

$n=0.98$

$\Delta H=-90.23 \mathrm{~kJ} \cdot \mathrm{mol}^{-1}$ $\Delta S=-98.03 \mathrm{~J} \cdot \mathrm{mol}^{-1} \cdot \mathrm{K}^{-1}$

Time (min)

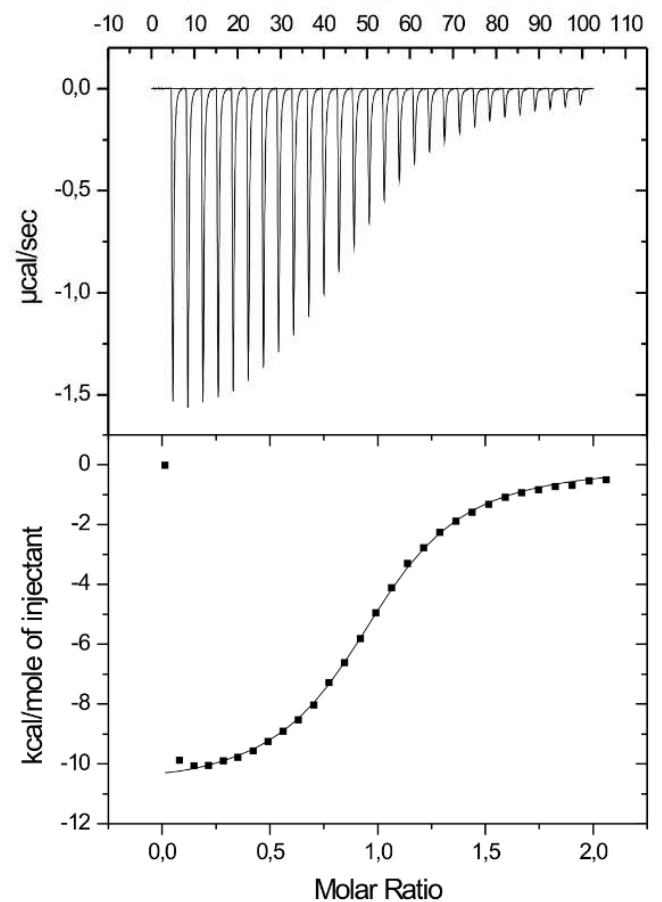

Figure S35 ITC data for the guest $\mathbf{5 b}$ and $\beta-\mathrm{CD}$ and CB7 


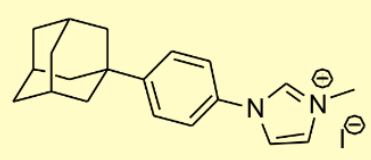

10

$10+\beta C D \underset{\mathrm{H}_{2} \mathrm{O}}{\stackrel{30^{\circ} \mathrm{C}}{\longrightarrow}} \quad 10 @ \beta C D$

syringe: $c(\mathbf{1 0})=1.65 \mathrm{mM}$ cell: $c(\beta C D)=0.17 \mathrm{mM}$

$K=8.68 \cdot 10^{5} \mathrm{M}^{-1}$ $n=0.98$

$\Delta H=-36.29 \mathrm{~kJ} \cdot \mathrm{mol}^{-1}$ $\Delta S=-6.03 \mathrm{~J} \cdot \mathrm{mol}^{-1} \cdot \mathrm{K}^{-1}$

Time (min)

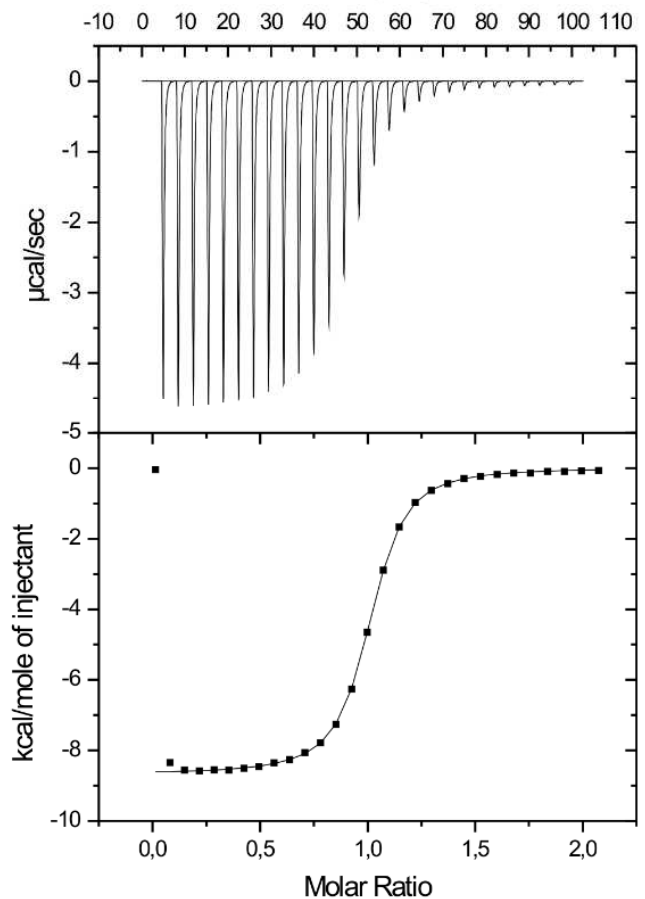

$10+\mathrm{CB} 7 \underset{\mathrm{H}_{2} \mathrm{O}}{\stackrel{30^{\circ} \mathrm{C}}{\longrightarrow}} 10 @ \mathrm{CB} 7$

syringe: $c(10)=0.58 \mathrm{mM}$ cell: $c(\mathrm{CB} 7)=0.06 \mathrm{mM}$ $c($ HeMelmCl $)=3.74 \mathrm{mM}$

$K=2.97 \cdot 10^{10} \mathrm{M}^{-1}$ $n=0.83$

$\Delta H=-84.86 \mathrm{~kJ} \cdot \mathrm{mol}^{-1}$ $\Delta S=-79.32 \mathrm{~J} \cdot \mathrm{mol}^{-1} \cdot \mathrm{K}^{-1}$

Time (min)

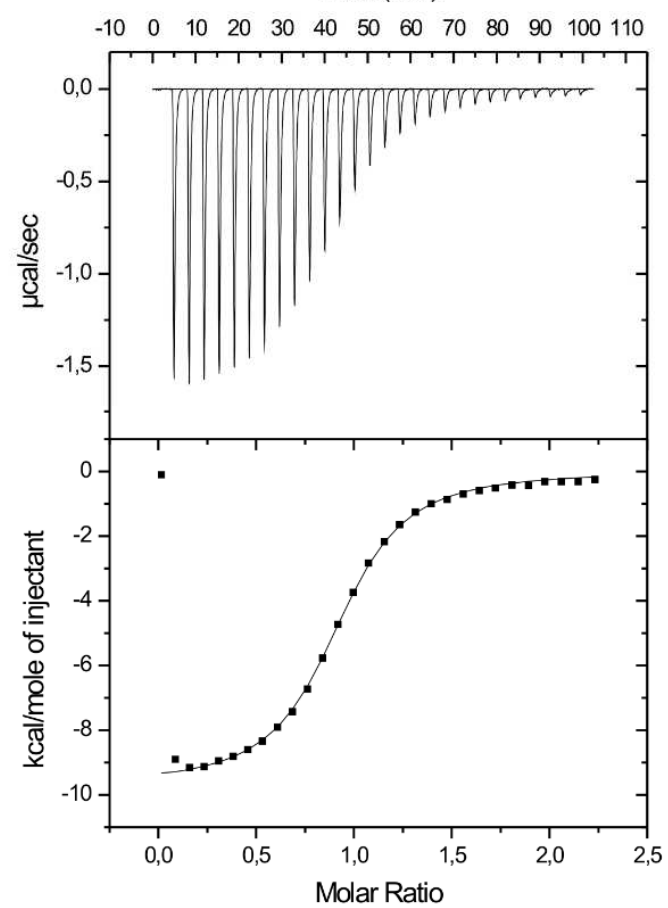

Figure S36 ITC data for the guest 10 and $\beta-C D$ and CB7 


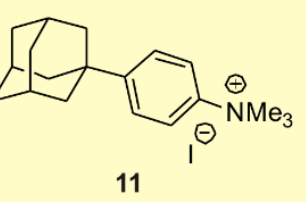

$11+\beta C D \underset{\mathrm{H}_{2} \mathrm{O}}{\stackrel{30^{\circ} \mathrm{C}}{\longrightarrow}} 11 @ \beta C D$

syringe: $c(11)=0.56 \mathrm{mM}$ cell: $c(\beta C D)=0.05 \mathrm{mM}$

$K=7.61 \cdot 10^{5} \mathrm{M}^{-1}$

$n=1.02$

$\Delta H=-34.24 \mathrm{~kJ} \cdot \mathrm{mol}^{-1}$

$\Delta S=-0.34 \mathrm{~J} \cdot \mathrm{mol}^{-1} \cdot \mathrm{K}^{-1}$

Time (min)

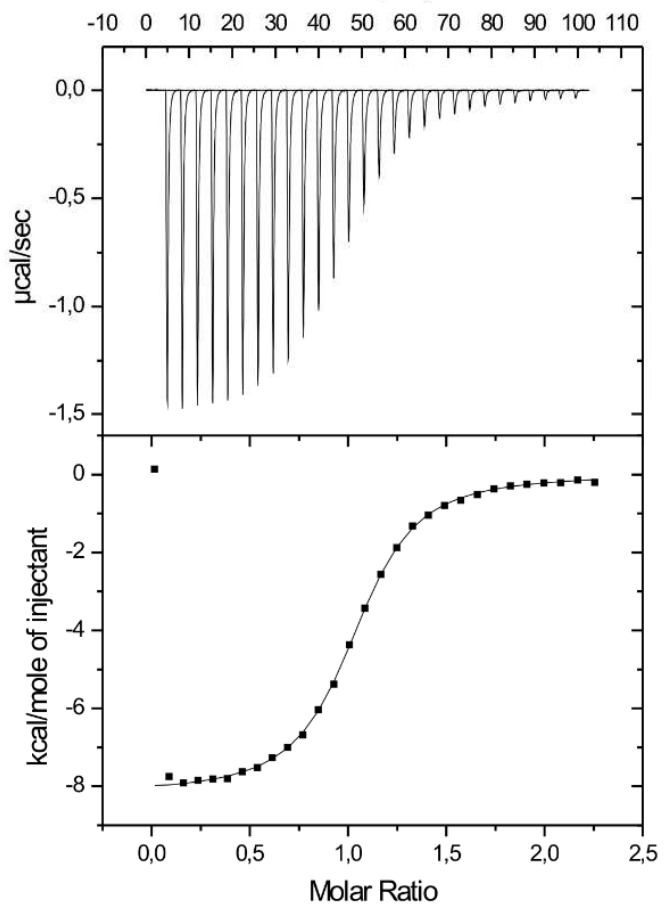

$11+\mathrm{CB} 7 \underset{\mathrm{H}_{2} \mathrm{O}}{\stackrel{30^{\circ} \mathrm{C}}{\longrightarrow}} 11 @ \mathrm{CB} 7$

syringe: $c(11)=0.46 \mathrm{mM}$ cell: $c(\mathrm{CB} 7)=0.06 \mathrm{mM}$ $c($ HeMelmCl $)=1.36 \mathrm{mM}$

$K=3.27 \cdot 10^{10} \mathrm{M}^{-1}$

$n=0.91$

$\Delta H=-81.01 \mathrm{~kJ} \cdot \mathrm{mol}^{-1}$ $\Delta S=-65.81 \mathrm{~J} \cdot \mathrm{mol}^{-1} \cdot \mathrm{K}^{-1}$

Time (min)

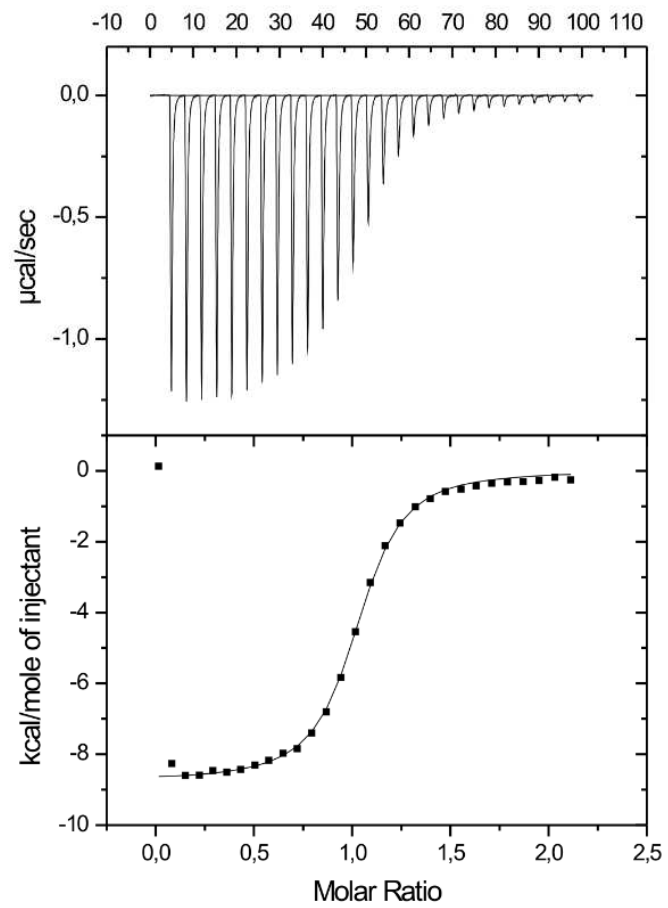

Figure S37 ITC data for the guest 11 and $\beta-C D$ and CB7 


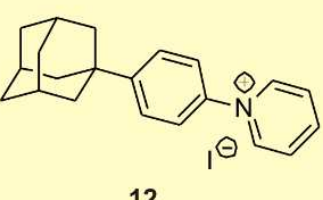

12

$12+\beta C D \underset{\mathrm{H}_{2} \mathrm{O}}{\stackrel{30^{\circ} \mathrm{C}}{\longrightarrow}} 12 @ \beta C D$

syringe: $c(12)=3.59 \mathrm{mM}$ cell: $c(\beta C D)=0.36 \mathrm{mM}$

$K=6.51 \cdot 10^{5} \mathrm{M}^{-1}$ $n=1.05$

$\Delta H=-33.94 \mathrm{~kJ} \cdot \mathrm{mol}^{-1}$ $\Delta S=-0.65 \mathrm{~J} \cdot \mathrm{mol}^{-1} \cdot \mathrm{K}^{-1}$

Time ( $\mathrm{min})$

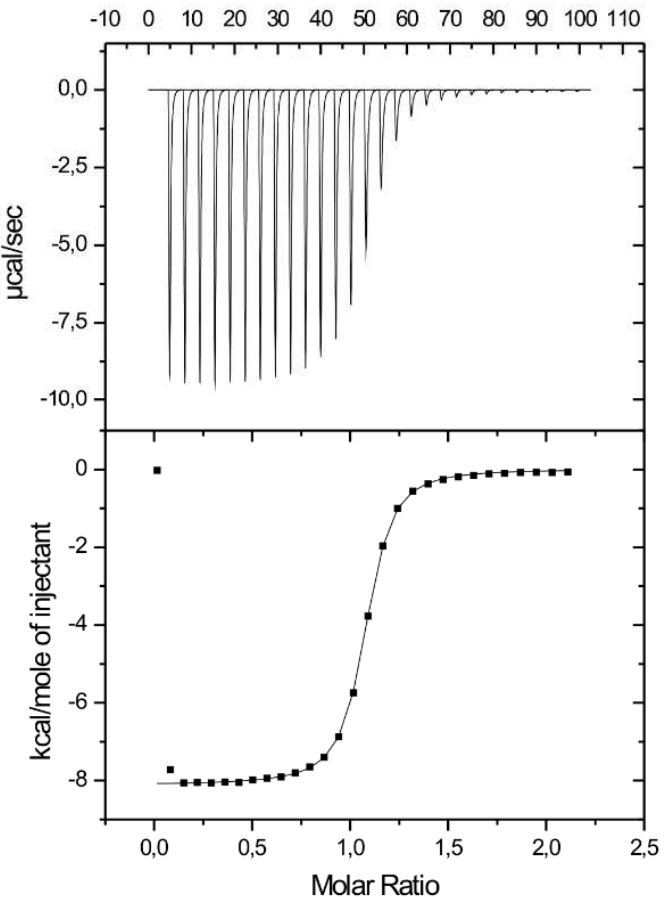

$12+\mathrm{CB} 7 \underset{\mathrm{H}_{2} \mathrm{O}}{\stackrel{30^{\circ} \mathrm{C}}{\longrightarrow}}$ 12@CB7

syringe: $c(\mathbf{1 2})=0.61 \mathrm{mM}$ cell: $c(\mathrm{CB} 7)=0.06 \mathrm{mM}$ $c($ HeMelmCl $)=4.12 \mathrm{mM}$

$K=4.25 \cdot 10^{10} \mathrm{M}^{-1}$

$n=1.05$

$\Delta H=-75.95 \mathrm{~kJ} \cdot \mathrm{mol}^{-1}$ $\Delta S=-46.94 \mathrm{~J} \cdot \mathrm{mol}^{-1} \cdot \mathrm{K}^{-1}$

Time $(\mathrm{min})$

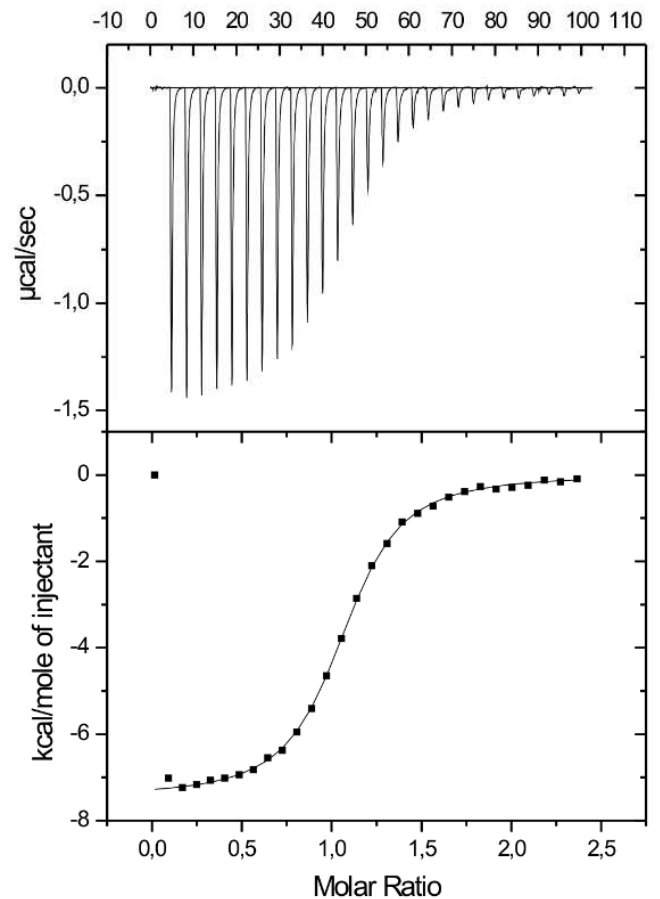

Figure S38 ITC data for the guest $\mathbf{1 2}$ and $\beta-\mathrm{CD}$ and CB7 

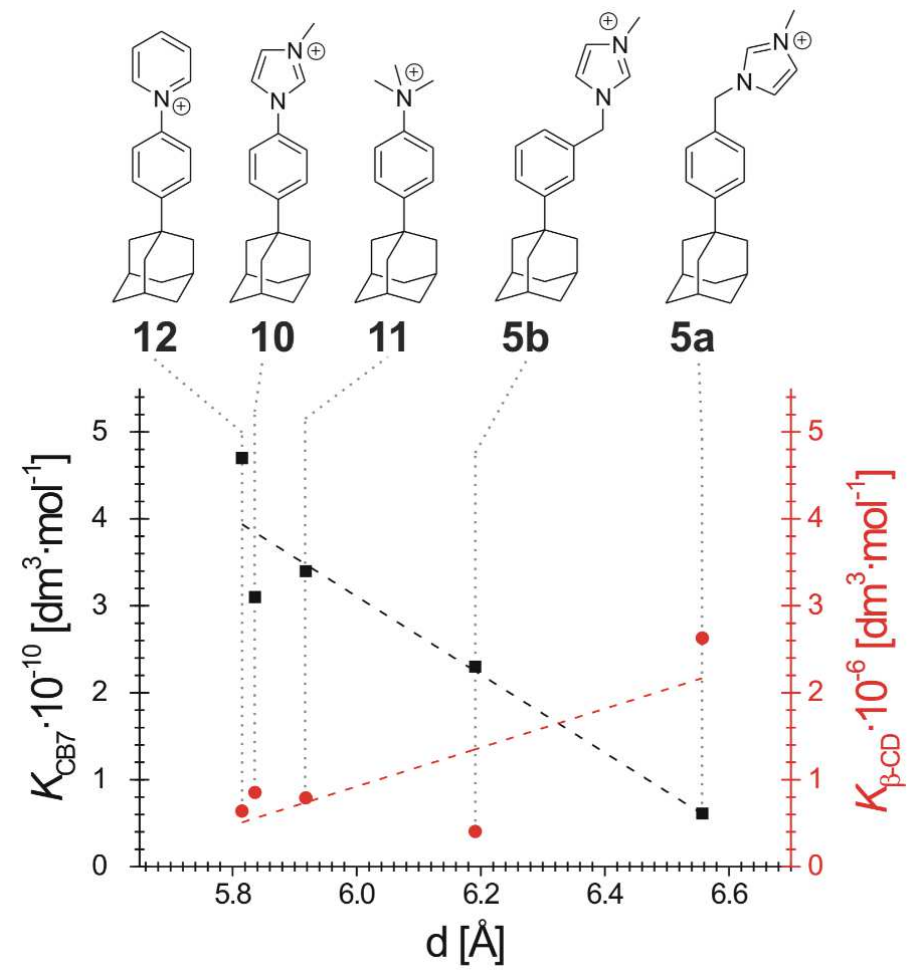

Figure S39 Plot of $K$ values against a distance $d$. The $d$ values were obtained from optimised molecular models (Avogadro, MMFF94) as point-to-point distances from adamantane $\mathrm{C} 1$ atom to $\mathrm{N}$-atom adjacent to the phenyl ring.

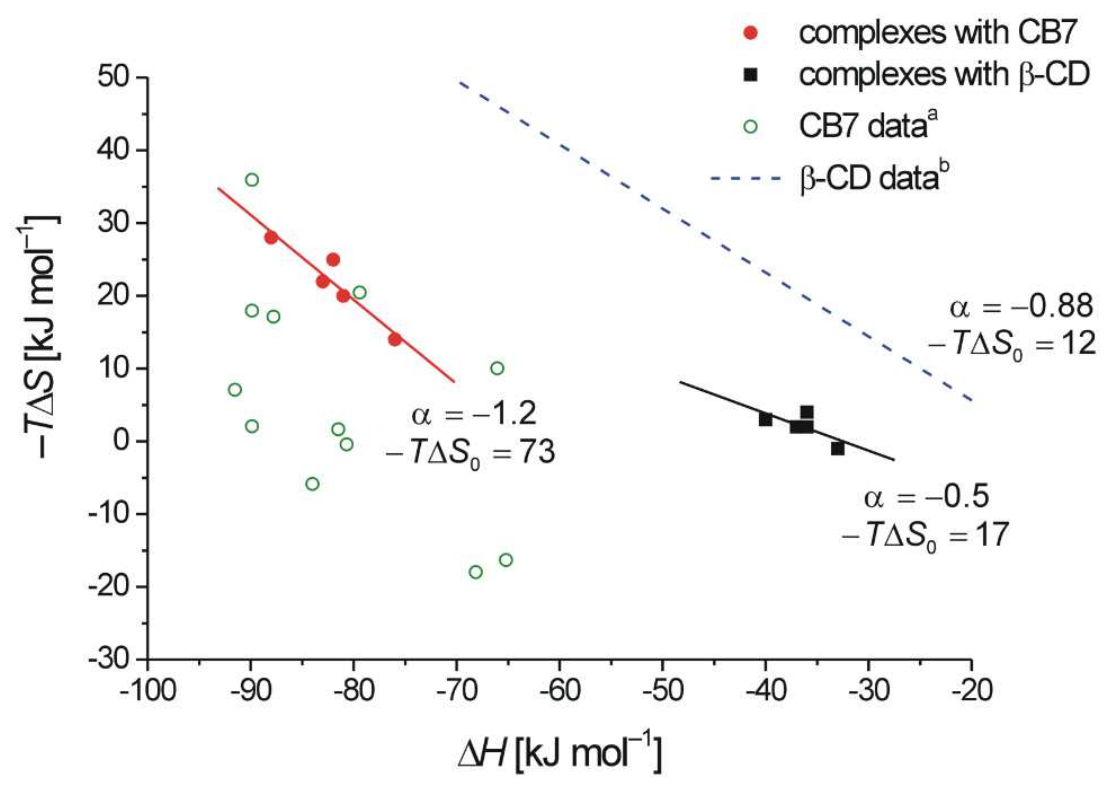

Figure S40 Enthalpy-entropy scatter plot for the guests 5a, 5b, 10, 11, and $\mathbf{1 2}$ with CB7 (red dots) and $\beta$-CD (black squares). ${ }^{a}$ Green hollow circles represents data for high-affinity CB7-guest pairs described in literature. ${ }^{1}$ blue dashed line is linear regression plot through data for 1070 complexes of natural cyclodextrins according to Rekharsky and Inoue. ${ }^{2}$ The slope $\alpha$ and intercept $T \Delta S_{0}$ are given in terms of equation $T \Delta S=\alpha \cdot \Delta H+T \Delta S_{0}$. 
Crystal data and structure refinement of compounds $\mathbf{3 b}, \mathbf{5 b}$

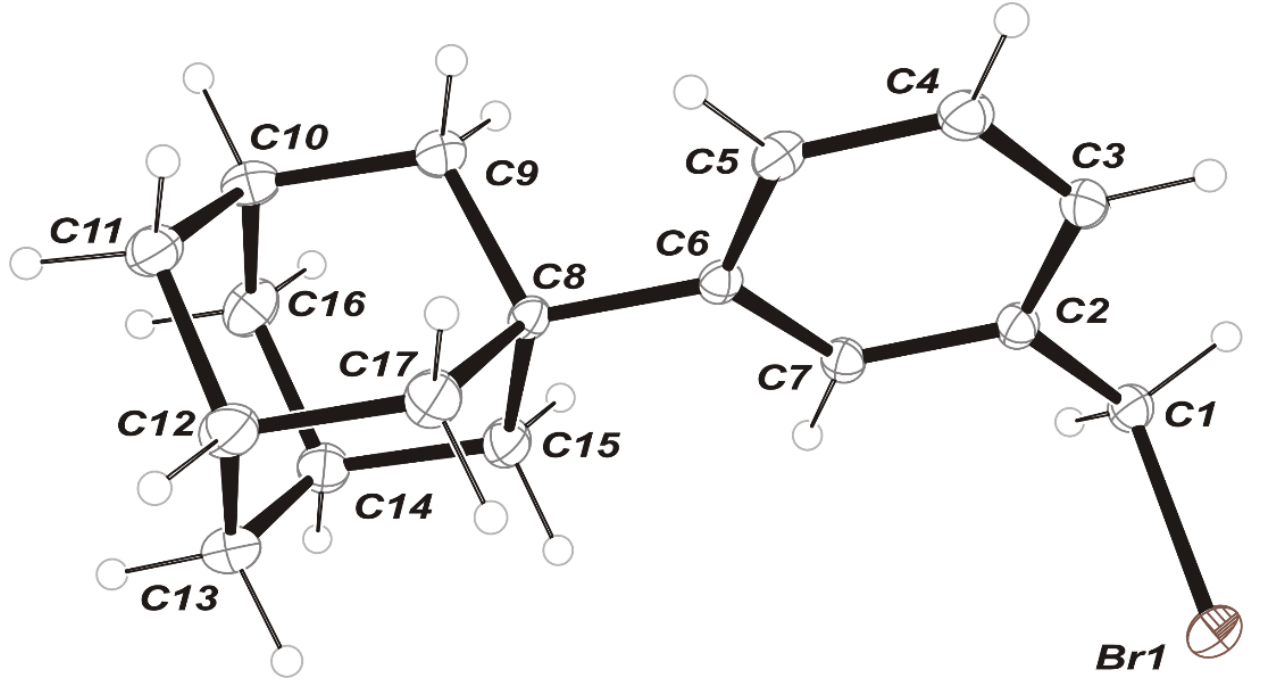

Figure S41 ORTEP diagram drawn with $40 \%$ ellipsoid probability for non-H atoms of the crystal structure of compound $\mathbf{3 b}$.

11

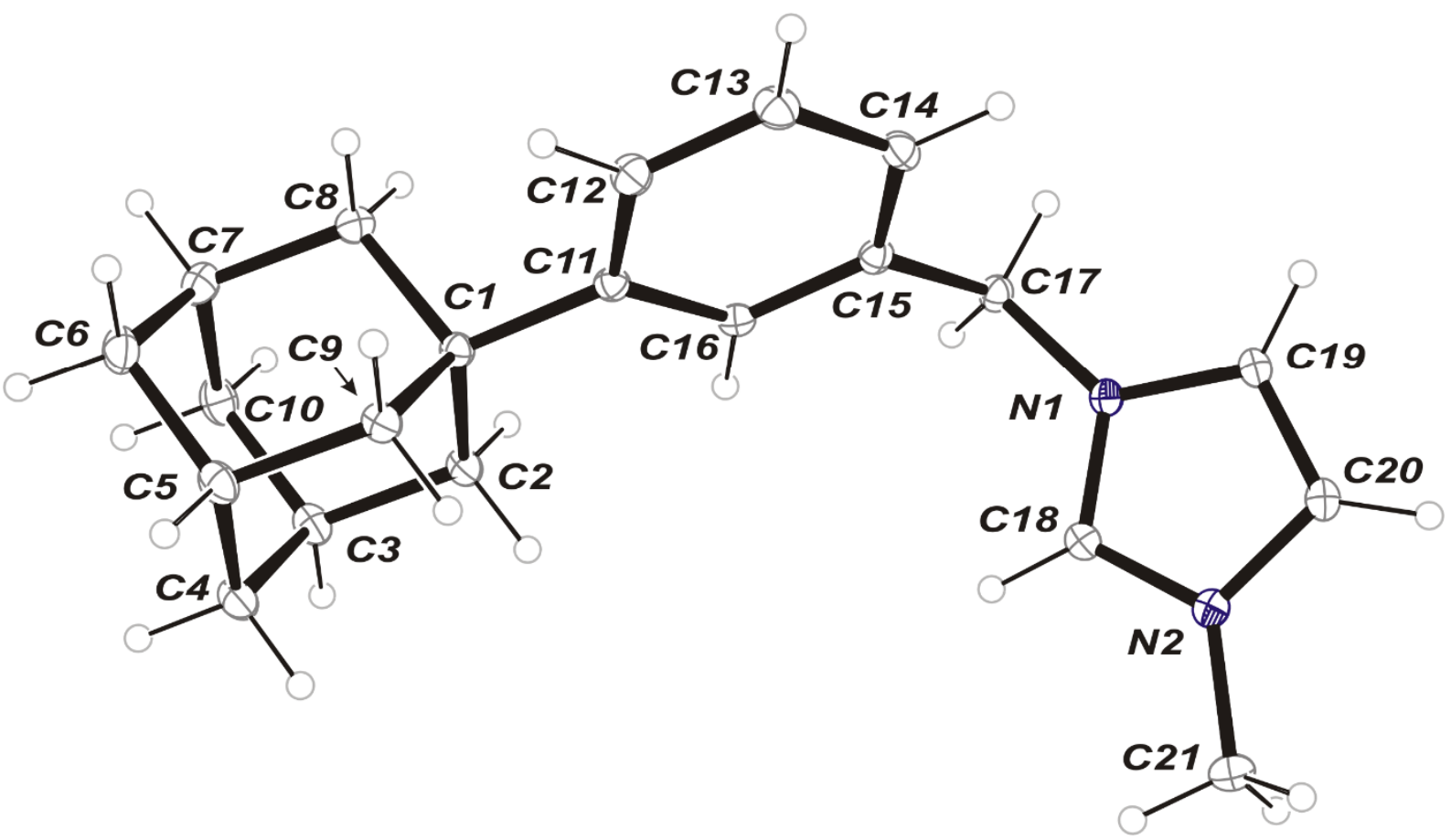

Figure S42 ORTEP diagram drawn with $40 \%$ ellipsoid probability for non-H atoms of the crystal structure of compound $\mathbf{5 b}$. 
Table S1: Crystal data and structure refinement of compounds $\mathbf{3 b}$ and $\mathbf{5 b}$.

\begin{tabular}{|c|c|c|}
\hline Compound & $3 \mathbf{b}$ & $\mathbf{5 b}$ \\
\hline CCDC deposition number & 1956556 & 1956557 \\
\hline Empirical formula & $\mathrm{C}_{17} \mathrm{H}_{21} \mathrm{Br}$ & $\mathrm{C}_{21} \mathrm{H}_{27} \mathrm{IN}_{2}$ \\
\hline Formula weight $\left(\mathrm{g} \cdot \mathrm{mol}^{-1}\right)$ & 305.25 & 434.34 \\
\hline Colour; shape & Colourless; block & Colourless; plate \\
\hline Crystal size & $0.20 \times 0.15 \times 0.15$ & $0.20 \times 0.15 \times 0.05$ \\
\hline Measured temperature $(\mathrm{K})$ & $120(2)$ & $120(2)$ \\
\hline Crystal system & Triclinic & Monoclinic \\
\hline Space group & $P \overline{1}$ & $\mathrm{P} 21 / \mathrm{c}$ \\
\hline \multirow[t]{6}{*}{ Unit cell dimensions $\left(\AA,^{\circ}\right)$} & $a=7.2323(3)$ & $a=17.6623(3)$ \\
\hline & $b=10.1462(3)$ & $b=8.31270(10)$ \\
\hline & $c=10.3654(4)$ & $c=13.6148(3)$ \\
\hline & $\alpha=98.048(3)$ & $\alpha=90$ \\
\hline & $\beta=100.941(4)$ & $\beta=104.077(2)$ \\
\hline & $\gamma=107.745(3)$ & $\gamma=90$ \\
\hline Volume $\left(\AA^{3}\right)$ & $695.16(5)$ & 1938.91(6) \\
\hline$Z$ & 2 & 4 \\
\hline$D_{x}\left(\mathrm{~g} \mathrm{~cm}^{-3}\right)$ & 1.458 & 1.488 \\
\hline$\mu\left(\mathrm{mm}^{-1}\right)$ & 2.937 & 1.656 \\
\hline Absorption correction & 0.83113 to 1.00000 & 0.89562 to 1.00000 \\
\hline$F(000)$ & 316 & 880 \\
\hline$\theta$ Range $\left(^{\circ}\right)$ & 2.047 to 29.884 & 2.724 to 25.348 \\
\hline Completeness to $\theta(\%)$ & 0.991 & 0.956 \\
\hline$h$ & $-9 \leq \mathrm{h} \leq 9$ & $-20 \leq \mathrm{h} \leq 21$ \\
\hline$k$ & $-12 \leq \mathrm{k} \leq 14$ & $-10 \leq \mathrm{k} \leq 10$ \\
\hline$l$ & $-14 \leq 1 \leq 13$ & $-16 \leq 1 \leq 12$ \\
\hline Reflections collected & 3262 & 3387 \\
\hline Reflections unique & $163[R(\mathrm{int})=0.0230]$ & $218[R(\mathrm{int})=0.0216]$ \\
\hline Unique reflections with $I \geq 2 \sigma(I)$ & 3044 & 3263 \\
\hline Number of parameters & 163 & 218 \\
\hline Goodness-of-fit on $F^{2}$ & 1.065 & 1.076 \\
\hline Final $R$ indices $[I \geq 2 \sigma(I)]$ & $R_{1}=0.0389, w R_{2}=0.1031$ & $R_{1}=0.0172, w R_{2}=0.0432$ \\
\hline$R$ indices (all data) & $R_{1}=0.0417, w R_{2}=0.1055$ & $R_{1}=0.0178, w R_{2}=0.0436$ \\
\hline Residual highest peak and deepest hole $\left(\mathrm{e} \cdot \AA^{-3}\right)$ & 1.353 and -0.437 & 0.641 and -0.258 \\
\hline
\end{tabular}


Atomic coordinates, geometrical parameters and atomic displacement parameters

Table S2: Geometric parameters $\left(\AA{ }^{\circ}{ }^{\circ}\right)$ for compound $\mathbf{3 b}$.

\begin{tabular}{|c|c|c|c|}
\hline $\mathrm{Br} 1-\mathrm{C} 1$ & $1.975(3)$ & $\mathrm{C} 10-\mathrm{C} 16$ & $1.511(4)$ \\
\hline $\mathrm{C} 1-\mathrm{C} 2$ & $1.493(4)$ & $\mathrm{C} 10-\mathrm{C} 11$ & $1.519(4)$ \\
\hline $\mathrm{C} 1-\mathrm{H} 1 \mathrm{~A}$ & 0.9900 & $\mathrm{C} 10-\mathrm{H} 10$ & 1.0000 \\
\hline $\mathrm{C} 1-\mathrm{H} 1 \mathrm{~B}$ & 0.9900 & $\mathrm{C} 11-\mathrm{C} 12$ & $1.516(4)$ \\
\hline $\mathrm{C} 3-\mathrm{C} 4$ & $1.387(4)$ & $\mathrm{C} 11-\mathrm{H} 11 \mathrm{~A}$ & 0.9900 \\
\hline $\mathrm{C} 3-\mathrm{C} 2$ & $1.393(4)$ & $\mathrm{C} 11-\mathrm{H} 11 \mathrm{~B}$ & 0.9900 \\
\hline $\mathrm{C} 3-\mathrm{H} 3$ & 0.9500 & $\mathrm{C} 12-\mathrm{C} 13$ & $1.502(5)$ \\
\hline $\mathrm{C} 2-\mathrm{C} 7$ & $1.400(3)$ & $\mathrm{C} 12-\mathrm{C} 17$ & $1.569(4)$ \\
\hline $\mathrm{C} 4-\mathrm{C} 5$ & $1.390(4)$ & $\mathrm{C} 12-\mathrm{H} 12$ & 1.0000 \\
\hline $\mathrm{C} 4-\mathrm{H} 4$ & 0.9500 & $\mathrm{C} 13-\mathrm{C} 14$ & $1.523(5)$ \\
\hline $\mathrm{C} 6-\mathrm{C} 7$ & $1.392(3)$ & $\mathrm{C} 13-\mathrm{H} 13 \mathrm{~A}$ & 0.9900 \\
\hline $\mathrm{C} 6-\mathrm{C} 5$ & $1.403(3)$ & $\mathrm{C} 13-\mathrm{H} 13 \mathrm{~B}$ & 0.9900 \\
\hline $\mathrm{C} 6-\mathrm{C} 8$ & $1.528(3)$ & $\mathrm{C} 14-\mathrm{C} 16$ & $1.513(4)$ \\
\hline $\mathrm{C} 5-\mathrm{H} 5$ & 0.9500 & $\mathrm{C} 14-\mathrm{C} 15$ & $1.562(4)$ \\
\hline $\mathrm{C} 7-\mathrm{H} 7$ & 0.9500 & C14-H14 & 1.0000 \\
\hline $\mathrm{C} 9-\mathrm{C} 8$ & $1.534(3)$ & $\mathrm{C} 15-\mathrm{H} 15 \mathrm{~A}$ & 0.9900 \\
\hline $\mathrm{C} 9-\mathrm{C} 10$ & $1.564(4)$ & C15-H15B & 0.9900 \\
\hline C9-H9A & 0.9900 & $\mathrm{C} 16-\mathrm{H} 16 \mathrm{~A}$ & 0.9900 \\
\hline C9-H9B & 0.9900 & C16-H16B & 0.9900 \\
\hline $\mathrm{C} 8-\mathrm{C} 17$ & $1.541(3)$ & $\mathrm{C} 17-\mathrm{H} 17 \mathrm{~A}$ & 0.9900 \\
\hline $\mathrm{C} 8-\mathrm{C} 15$ & $1.558(3)$ & C17-H17B & 0.9900 \\
\hline $\mathrm{C} 11-\mathrm{C} 12-\mathrm{H} 12$ & 109.9 & $\mathrm{C} 14-\mathrm{C} 15-\mathrm{H} 15 \mathrm{~A}$ & 109.5 \\
\hline $\mathrm{C} 17-\mathrm{C} 12-\mathrm{H} 12$ & 109.9 & $\mathrm{C} 8-\mathrm{C} 15-\mathrm{H} 15 \mathrm{~B}$ & 109.5 \\
\hline $\mathrm{C} 12-\mathrm{C} 13-\mathrm{C} 14$ & $110.8(2)$ & $\mathrm{C} 14-\mathrm{C} 15-\mathrm{H} 15 \mathrm{~B}$ & 109.5 \\
\hline $\mathrm{C} 12-\mathrm{C} 13-\mathrm{H} 13 \mathrm{~A}$ & 109.5 & $\mathrm{H} 15 \mathrm{~A}-\mathrm{C} 15-\mathrm{H} 15 \mathrm{~B}$ & 108.1 \\
\hline $\mathrm{C} 14-\mathrm{C} 13-\mathrm{H} 13 \mathrm{~A}$ & 109.5 & $\mathrm{C} 10-\mathrm{C} 16-\mathrm{C} 14$ & $111.0(2)$ \\
\hline $\mathrm{C} 12-\mathrm{C} 13-\mathrm{H} 13 \mathrm{~B}$ & 109.5 & $\mathrm{C} 10-\mathrm{C} 16-\mathrm{H} 16 \mathrm{~A}$ & 109.4 \\
\hline $\mathrm{C} 14-\mathrm{C} 13-\mathrm{H} 13 \mathrm{~B}$ & 109.5 & $\mathrm{C} 14-\mathrm{C} 16-\mathrm{H} 16 \mathrm{~A}$ & 109.4 \\
\hline $\mathrm{H} 13 \mathrm{~A}-\mathrm{C} 13-\mathrm{H} 13 \mathrm{~B}$ & 108.1 & $\mathrm{C} 10-\mathrm{C} 16-\mathrm{H} 16 \mathrm{~B}$ & 109.4 \\
\hline $\mathrm{C} 16-\mathrm{C} 14-\mathrm{C} 13$ & $110.8(3)$ & $\mathrm{C} 14-\mathrm{C} 16-\mathrm{H} 16 \mathrm{~B}$ & 109.4 \\
\hline $\mathrm{C} 16-\mathrm{C} 14-\mathrm{C} 15$ & $109.1(2)$ & $\mathrm{H} 16 \mathrm{~A}-\mathrm{C} 16-\mathrm{H} 16 \mathrm{~B}$ & 108.0 \\
\hline $\mathrm{C} 13-\mathrm{C} 14-\mathrm{C} 15$ & $107.4(3)$ & $\mathrm{C} 8-\mathrm{C} 17-\mathrm{C} 12$ & $110.8(2)$ \\
\hline
\end{tabular}




\begin{tabular}{llll}
\hline C16-C14-H14 & 109.8 & C8-C17-H17A & 109.5 \\
C13-C14-H14 & 109.8 & C12-C17-H17A & 109.5 \\
C15-C14-H14 & 109.8 & C8-C17-H17B & 109.5 \\
C8-C15-C14 & $110.6(2)$ & C12-C17-H17B & 109.5 \\
C8-C15-H15A & 109.5 & H17A-C17-H17B & 109.5 \\
\hline
\end{tabular}

Table S3: Fractional atomic coordinates and isotropic or equivalent isotropic displacement parameters $\left(\AA^{2}\right)$ for compound $\mathbf{3 b}$.

\begin{tabular}{|c|c|c|c|c|}
\hline & $\mathrm{x}$ & $\mathrm{y}$ & $\mathrm{z}$ & $\mathrm{U}_{\mathrm{iso}} * / \mathrm{U}_{\mathrm{eq}}$ \\
\hline $\mathrm{Br} 1$ & $1.10071(4)$ & $0.82138(3)$ & $0.64311(2)$ & $0.02969(11)$ \\
\hline $\mathrm{C} 1$ & $0.8352(4)$ & $0.6786(3)$ & $0.6235(3)$ & $0.0271(5)$ \\
\hline $\mathrm{H} 1 \mathrm{~A}$ & 0.852428 & 0.596847 & 0.659900 & $0.032 *$ \\
\hline H1B & 0.757991 & 0.720208 & 0.675959 & $0.032 *$ \\
\hline $\mathrm{C} 3$ & $0.7297(4)$ & $0.5089(3)$ & $0.4009(3)$ & $0.0263(5)$ \\
\hline $\mathrm{H} 3$ & 0.808181 & 0.457181 & 0.438374 & $0.032 *$ \\
\hline $\mathrm{C} 2$ & $0.7215(4)$ & $0.6292(3)$ & $0.4795(3)$ & $0.0227(5)$ \\
\hline $\mathrm{C} 4$ & $0.6222(4)$ & $0.4653(3)$ & $0.2675(3)$ & $0.0284(5)$ \\
\hline $\mathrm{H} 4$ & 0.628527 & 0.384027 & 0.213178 & $0.034^{*}$ \\
\hline C6 & $0.4924(3)$ & $0.6600(3)$ & $0.2899(2)$ & $0.0204(4)$ \\
\hline C5 & $0.5051(4)$ & $0.5395(3)$ & $0.2125(3)$ & $0.0250(5)$ \\
\hline H5 & 0.432539 & 0.508063 & 0.120908 & $0.030^{*}$ \\
\hline $\mathrm{C} 7$ & $0.6025(4)$ & $0.7027(3)$ & $0.4233(2)$ & $0.0217(5)$ \\
\hline $\mathrm{H} 7$ & 0.596740 & 0.784173 & 0.477726 & $0.026^{*}$ \\
\hline C9 & $0.1410(4)$ & $0.6382(3)$ & $0.1808(3)$ & $0.0292(5)$ \\
\hline H9A & 0.094479 & 0.604656 & 0.258081 & $0.035^{*}$ \\
\hline H9B & 0.133506 & 0.554576 & 0.115277 & $0.035^{*}$ \\
\hline $\mathrm{C} 8$ & $0.3591(3)$ & $0.7384(3)$ & $0.2295(2)$ & $0.0197(4)$ \\
\hline $\mathrm{C} 10$ & $0.0002(4)$ & $0.7127(3)$ & $0.1132(3)$ & $0.0312(6)$ \\
\hline H10 & -0.140241 & 0.644746 & 0.079941 & $0.037 *$ \\
\hline $\mathrm{C} 11$ & $0.0792(5)$ & $0.7636(3)$ & $-0.0033(3)$ & $0.0332(6)$ \\
\hline H11A & 0.082571 & 0.681925 & -0.066317 & $0.040^{*}$ \\
\hline H11B & -0.012364 & 0.805180 & -0.052609 & $0.040^{*}$ \\
\hline $\mathrm{C} 12$ & $0.2877(5)$ & $0.8732(3)$ & $0.0456(3)$ & $0.0368(7)$ \\
\hline H12 & 0.333540 & 0.909414 & -0.031853 & $0.040^{*}$ \\
\hline $\mathrm{C} 13$ & $0.2967(5)$ & $0.9943(3)$ & $0.1510(4)$ & $0.0409(7)$ \\
\hline
\end{tabular}




\begin{tabular}{lllll}
\hline H13A & 0.214279 & 1.047187 & 0.110777 & $0.049^{*}$ \\
H13B & 0.436706 & 1.059956 & 0.184576 & $0.049^{*}$ \\
C14 & $0.2202(5)$ & $0.9410(3)$ & $0.2678(3)$ & $0.0344(6)$ \\
H14 & 0.222646 & 1.022518 & 0.335497 & $0.041^{*}$ \\
C15 & $0.3629(4)$ & $0.8669(3)$ & $0.3331(3)$ & $0.0300(6)$ \\
H15A & 0.501185 & 0.935511 & 0.364847 & $0.036^{*}$ \\
H15B & 0.320330 & 0.833913 & 0.411710 & $0.036^{*}$ \\
C16 & $0.0097(5)$ & $0.8350(4)$ & $0.2189(3)$ & $0.0380(7)$ \\
H16A & -0.034818 & 0.798796 & 0.295727 & $0.046^{*}$ \\
H16B & -0.082356 & 0.882455 & 0.180659 & $0.046^{*}$ \\
C17 & $0.4281(4)$ & $0.7963(3)$ & $0.1105(3)$ & $0.0317(6)$ \\
H17A & 0.426534 & 0.717305 & 0.041949 & $0.038^{*}$ \\
H17B & 0.567208 & 0.863691 & 0.142306 & $0.038^{*}$ \\
\hline
\end{tabular}

Table S4: Atomic displacement parameters $\left(\AA^{2}\right)$ for compound $\mathbf{3 b}$.

\begin{tabular}{lllllll}
\hline & $\mathrm{U}^{11}$ & $\mathrm{U}^{22}$ & $\mathrm{U}^{33}$ & $\mathrm{U}^{23}$ & $\mathrm{U}^{13}$ & $\mathrm{U}^{12}$ \\
\hline $\mathrm{Br} 1$ & $0.02451(16)$ & $0.03336(17)$ & $0.02539(16)$ & $0.00198(11)$ & $0.00233(11)$ & $0.00584(11)$ \\
$\mathrm{C} 1$ & $0.0255(12)$ & $0.0287(13)$ & $0.0263(12)$ & $0.0126(10)$ & $0.0036(10)$ & $0.0069(10)$ \\
$\mathrm{C} 3$ & $0.0216(11)$ & $0.0238(12)$ & $0.0342(14)$ & $0.0080(10)$ & $0.0060(10)$ & $0.0083(10)$ \\
$\mathrm{C} 2$ & $0.0197(11)$ & $0.0246(12)$ & $0.0245(12)$ & $0.0098(9)$ & $0.0050(9)$ & $0.0067(9)$ \\
$\mathrm{C} 4$ & $0.0241(12)$ & $0.0235(12)$ & $0.0355(14)$ & $-0.0002(10)$ & $0.0067(11)$ & $0.0082(10)$ \\
$\mathrm{C} 5$ & $0.0206(11)$ & $0.0265(12)$ & $0.0241(12)$ & $0.0002(10)$ & $0.0035(9)$ & $0.0059(10)$ \\
$\mathrm{C} 7$ & $0.0217(11)$ & $0.0225(11)$ & $0.0217(11)$ & $0.0067(9)$ & $0.0063(9)$ & $0.0073(9)$ \\
$\mathrm{C} 9$ & $0.0252(12)$ & $0.0268(13)$ & $0.0319(13)$ & $0.0070(11)$ & $0.0046(11)$ & $0.0048(10)$ \\
$\mathrm{C} 8$ & $0.0179(10)$ & $0.0226(11)$ & $0.0172(10)$ & $0.0047(8)$ & $0.0033(8)$ & $0.0054(9)$ \\
$\mathrm{C} 10$ & $0.0243(12)$ & $0.0276(13)$ & $0.0357(14)$ & $0.0046(11)$ & $0.0075(11)$ & $0.0013(10)$ \\
$\mathrm{C} 11$ & $0.0321(14)$ & $0.0315(14)$ & $0.0269(13)$ & $0.0057(11)$ & $0.0021(11)$ & $0.0015(12)$ \\
$\mathrm{C} 12$ & $0.0324(15)$ & $0.0397(16)$ & $0.0291(14)$ & $0.0146(12)$ & $0.0048(12)$ & $-0.0021(12)$ \\
$\mathrm{C} 13$ & $0.0316(15)$ & $0.0286(14)$ & $0.0507(19)$ & $0.0082(13)$ & $-0.0017(14)$ & $0.0009(12)$ \\
$\mathrm{C} 14$ & $0.0409(16)$ & $0.0238(13)$ & $0.0331(14)$ & $-0.0017(11)$ & $-0.0001(12)$ & $0.0126(12)$ \\
$\mathrm{C} 15$ & $0.0292(13)$ & $0.0291(13)$ & $0.0289(13)$ & $0.0024(11)$ & $0.0026(11)$ & $0.0105(11)$ \\
$\mathrm{C} 16$ & $0.0282(14)$ & $0.0458(17)$ & $0.0370(15)$ & $0.0044(13)$ & $0.0062(12)$ & $0.0117(13)$ \\
$\mathrm{C} 17$ & $0.0284(13)$ & $0.0369(15)$ & $0.0291(13)$ & $0.0110(11)$ & $0.0086(11)$ & $0.0074(11)$ \\
\hline & & & & & &
\end{tabular}


Table S5: Geometric parameters $\left(\AA \mathrm{A}^{\circ}\right)$ for compound $\mathbf{5 b}$.

\begin{tabular}{|c|c|c|c|}
\hline $\mathrm{N} 1-\mathrm{C} 18$ & $1.327(2)$ & $\mathrm{C} 7-\mathrm{H} 7$ & 1.0000 \\
\hline $\mathrm{N} 1-\mathrm{C} 19$ & $1.382(2)$ & $\mathrm{C} 8-\mathrm{H} 8 \mathrm{~A}$ & 0.9900 \\
\hline $\mathrm{N} 1-\mathrm{C} 17$ & $1.482(2)$ & $\mathrm{C} 8-\mathrm{H} 8 \mathrm{AB}$ & 0.9900 \\
\hline N2- $\mathrm{C} 18$ & $1.331(2)$ & $\mathrm{C} 9-\mathrm{H} 9 \mathrm{~A}$ & 0.9900 \\
\hline $\mathrm{N} 2-\mathrm{C} 20$ & $1.387(2)$ & C9-H9AB & 0.9900 \\
\hline $\mathrm{N} 2-\mathrm{C} 21$ & $1.468(2)$ & $\mathrm{C} 10-\mathrm{H} 10 \mathrm{~A}$ & 0.9900 \\
\hline $\mathrm{C} 1-\mathrm{C} 11$ & $1.529(2)$ & $\mathrm{C} 10-\mathrm{H} 10 \mathrm{~B}$ & 0.9900 \\
\hline $\mathrm{C} 1-\mathrm{C} 2$ & $1.537(2)$ & $\mathrm{C} 11-\mathrm{C} 12$ & $1.401(3)$ \\
\hline $\mathrm{C} 1-\mathrm{C} 8$ & $1.548(2)$ & $\mathrm{C} 11-\mathrm{C} 16$ & $1.403(2)$ \\
\hline $\mathrm{C} 1-\mathrm{C} 9$ & $1.551(2)$ & $\mathrm{C} 12-\mathrm{C} 13$ & $1.387(3)$ \\
\hline $\mathrm{C} 2-\mathrm{C} 3$ & $1.540(2)$ & $\mathrm{C} 12-\mathrm{H} 12$ & 0.9500 \\
\hline $\mathrm{C} 2-\mathrm{H} 2 \mathrm{~A}$ & 0.9900 & $\mathrm{C} 13-\mathrm{C} 14$ & $1.393(3)$ \\
\hline $\mathrm{C} 2-\mathrm{H} 2 \mathrm{AB}$ & 0.9900 & $\mathrm{C} 13-\mathrm{H} 13$ & 0.9500 \\
\hline $\mathrm{C} 3-\mathrm{C} 10$ & $1.530(3)$ & $\mathrm{C} 14-\mathrm{C} 15$ & $1.395(2)$ \\
\hline $\mathrm{C} 3-\mathrm{C} 4$ & $1.537(2)$ & $\mathrm{C} 14-\mathrm{H} 14$ & 0.9500 \\
\hline $\mathrm{C} 3-\mathrm{H} 3$ & 1.0000 & $\mathrm{C} 15-\mathrm{C} 16$ & $1.395(2)$ \\
\hline $\mathrm{C} 4-\mathrm{C} 5$ & $1.528(3)$ & $\mathrm{C} 15-\mathrm{C} 17$ & $1.515(2)$ \\
\hline $\mathrm{C} 4-\mathrm{H} 4 \mathrm{~A}$ & 0.9900 & $\mathrm{C} 16-\mathrm{H} 16$ & 0.9500 \\
\hline $\mathrm{C} 4-\mathrm{H} 4 \mathrm{AB}$ & 0.9900 & C17-H17A & 0.9900 \\
\hline $\mathrm{C} 5-\mathrm{C} 6$ & $1.533(3)$ & C17-H17B & 0.9900 \\
\hline $\mathrm{C} 5-\mathrm{C} 9$ & $1.538(2)$ & $\mathrm{C} 18-\mathrm{H} 18$ & 0.9500 \\
\hline $\mathrm{C} 5-\mathrm{H} 5$ & 1.0000 & $\mathrm{C} 19-\mathrm{C} 20$ & $1.353(3)$ \\
\hline $\mathrm{C} 6-\mathrm{C} 7$ & $1.538(3)$ & $\mathrm{C} 19-\mathrm{H} 19$ & 0.9500 \\
\hline C6-H6A & 0.9900 & $\mathrm{C} 20-\mathrm{H} 20$ & 0.9500 \\
\hline C6- & 0.9900 & $\mathrm{C} 21-\mathrm{H} 21 \mathrm{~A}$ & 0.9800 \\
\hline $\mathrm{C} 7-\mathrm{C} 10$ & $1.533(3)$ & $\mathrm{C} 21-\mathrm{H} 21 \mathrm{~B}$ & 0.9800 \\
\hline $\mathrm{C} 7-\mathrm{C} 8$ & $1.537(3)$ & $\mathrm{C} 21-\mathrm{H} 21 \mathrm{C}$ & 0.9800 \\
\hline $\mathrm{C} 18-\mathrm{N} 1-\mathrm{C} 19$ & $108.75(14)$ & $\mathrm{H} 8 \mathrm{~A}-\mathrm{C} 8-\mathrm{H} 8 \mathrm{AB}$ & 108.0 \\
\hline $\mathrm{C} 18-\mathrm{N} 1-\mathrm{C} 17$ & $124.81(14)$ & $\mathrm{C} 5-\mathrm{C} 9-\mathrm{C} 1$ & $110.72(14)$ \\
\hline $\mathrm{C} 19-\mathrm{N} 1-\mathrm{C} 17$ & $126.32(14)$ & $\mathrm{C} 5-\mathrm{C} 9-\mathrm{H} 9 \mathrm{~A}$ & 109.5 \\
\hline $\mathrm{C} 18-\mathrm{N} 2-\mathrm{C} 20$ & $108.35(15)$ & $\mathrm{C} 1-\mathrm{C} 9-\mathrm{H} 9 \mathrm{~A}$ & 109.5 \\
\hline $\mathrm{C} 18-\mathrm{N} 2-\mathrm{C} 21$ & $125.12(15)$ & $\mathrm{C} 5-\mathrm{C} 9-\mathrm{H} 9 \mathrm{AB}$ & 109.5 \\
\hline $\mathrm{C} 20-\mathrm{N} 2-\mathrm{C} 21$ & $126.50(14)$ & $\mathrm{C} 1-\mathrm{C} 9-\mathrm{H} 9 \mathrm{AB}$ & 109.5 \\
\hline
\end{tabular}




\begin{tabular}{|c|c|c|c|}
\hline $\mathrm{C} 11-\mathrm{C} 1-\mathrm{C} 2$ & $113.15(13)$ & H9A-C9-H9AB & 108.1 \\
\hline $\mathrm{C} 11-\mathrm{C} 1-\mathrm{C} 8$ & $109.13(14)$ & $\mathrm{C} 3-\mathrm{C} 10-\mathrm{C} 7$ & $109.54(15)$ \\
\hline $\mathrm{C} 2-\mathrm{C} 1-\mathrm{C} 8$ & $108.00(14)$ & $\mathrm{C} 3-\mathrm{C} 10-\mathrm{H} 10 \mathrm{~A}$ & 109.8 \\
\hline $\mathrm{C} 11-\mathrm{C} 1-\mathrm{C} 9$ & $110.01(13)$ & $\mathrm{C} 7-\mathrm{C} 10-\mathrm{H} 10 \mathrm{~A}$ & 109.8 \\
\hline $\mathrm{C} 2-\mathrm{C} 1-\mathrm{C} 9$ & $107.93(14)$ & $\mathrm{C} 3-\mathrm{C} 10-\mathrm{H} 10 \mathrm{~B}$ & 109.8 \\
\hline $\mathrm{C} 8-\mathrm{C} 1-\mathrm{C} 9$ & $108.50(13)$ & $\mathrm{C} 7-\mathrm{C} 10-\mathrm{H} 10 \mathrm{~B}$ & 109.8 \\
\hline $\mathrm{C} 1-\mathrm{C} 2-\mathrm{C} 3$ & $110.76(14)$ & $\mathrm{H} 10 \mathrm{~A}-\mathrm{C} 10-\mathrm{H} 10 \mathrm{~B}$ & 108.2 \\
\hline $\mathrm{C} 1-\mathrm{C} 2-\mathrm{H} 2 \mathrm{~A}$ & 109.5 & $\mathrm{C} 12-\mathrm{C} 11-\mathrm{C} 16$ & $117.32(16)$ \\
\hline $\mathrm{C} 3-\mathrm{C} 2-\mathrm{H} 2 \mathrm{~A}$ & 109.5 & $\mathrm{C} 12-\mathrm{C} 11-\mathrm{C} 1$ & $120.09(15)$ \\
\hline $\mathrm{C} 1-\mathrm{C} 2-\mathrm{H} 2 \mathrm{AB}$ & 109.5 & $\mathrm{C} 16-\mathrm{C} 11-\mathrm{C} 1$ & $122.57(16)$ \\
\hline $\mathrm{C} 3-\mathrm{C} 2-\mathrm{H} 2 \mathrm{AB}$ & 109.5 & $\mathrm{C} 13-\mathrm{C} 12-\mathrm{C} 11$ & $121.63(16)$ \\
\hline $\mathrm{H} 2 \mathrm{~A}-\mathrm{C} 2-\mathrm{H} 2 \mathrm{AB}$ & 108.1 & $\mathrm{C} 13-\mathrm{C} 12-\mathrm{H} 12$ & 119.2 \\
\hline $\mathrm{C} 10-\mathrm{C} 3-\mathrm{C} 4$ & $109.41(15)$ & $\mathrm{C} 11-\mathrm{C} 12-\mathrm{H} 12$ & 119.2 \\
\hline $\mathrm{C} 10-\mathrm{C} 3-\mathrm{C} 2$ & $109.80(15)$ & $\mathrm{C} 12-\mathrm{C} 13-\mathrm{C} 14$ & $120.26(17)$ \\
\hline $\mathrm{C} 4-\mathrm{C} 3-\mathrm{C} 2$ & $109.22(14)$ & $\mathrm{C} 12-\mathrm{C} 13-\mathrm{H} 13$ & 119.9 \\
\hline $\mathrm{C} 10-\mathrm{C} 3-\mathrm{H} 3$ & 109.5 & $\mathrm{C} 14-\mathrm{C} 13-\mathrm{H} 13$ & 119.9 \\
\hline $\mathrm{C} 4-\mathrm{C} 3-\mathrm{H} 3$ & 109.5 & $\mathrm{C} 13-\mathrm{C} 14-\mathrm{C} 15$ & $119.33(16)$ \\
\hline $\mathrm{C} 2-\mathrm{C} 3-\mathrm{H} 3$ & 109.5 & $\mathrm{C} 13-\mathrm{C} 14-\mathrm{H} 14$ & 120.3 \\
\hline $\mathrm{C} 5-\mathrm{C} 4-\mathrm{C} 3$ & 109.62(15) & $\mathrm{C} 15-\mathrm{C} 14-\mathrm{H} 14$ & 120.3 \\
\hline $\mathrm{C} 5-\mathrm{C} 4-\mathrm{H} 4 \mathrm{~A}$ & 109.7 & $\mathrm{C} 16-\mathrm{C} 15-\mathrm{C} 14$ & $119.93(16)$ \\
\hline $\mathrm{C} 3-\mathrm{C} 4-\mathrm{H} 4 \mathrm{~A}$ & 109.7 & $\mathrm{C} 16-\mathrm{C} 15-\mathrm{C} 17$ & $119.46(16)$ \\
\hline $\mathrm{C} 5-\mathrm{C} 4-\mathrm{H} 4 \mathrm{AB}$ & 109.7 & $\mathrm{C} 14-\mathrm{C} 15-\mathrm{C} 17$ & $120.58(15)$ \\
\hline $\mathrm{C} 3-\mathrm{C} 4-\mathrm{H} 4 \mathrm{AB}$ & 109.7 & $\mathrm{C} 15-\mathrm{C} 16-\mathrm{C} 11$ & $121.51(17)$ \\
\hline $\mathrm{H} 4 \mathrm{~A}-\mathrm{C} 4-\mathrm{H} 4 \mathrm{AB}$ & 108.2 & $\mathrm{C} 15-\mathrm{C} 16-\mathrm{H} 16$ & 119.2 \\
\hline $\mathrm{C} 4-\mathrm{C} 5-\mathrm{C} 6$ & $109.90(15)$ & $\mathrm{C} 11-\mathrm{C} 16-\mathrm{H} 16$ & 119.2 \\
\hline $\mathrm{C} 4-\mathrm{C} 5-\mathrm{C} 9$ & $108.99(14)$ & $\mathrm{N} 1-\mathrm{C} 17-\mathrm{C} 15$ & $112.44(13)$ \\
\hline $\mathrm{C} 6-\mathrm{C} 5-\mathrm{C} 9$ & $109.42(15)$ & $\mathrm{N} 1-\mathrm{C} 17-\mathrm{H} 17 \mathrm{~A}$ & 109.1 \\
\hline $\mathrm{C} 4-\mathrm{C} 5-\mathrm{H} 5-$ & 109.5 & $\mathrm{C} 15-\mathrm{C} 17-\mathrm{H} 17 \mathrm{~A}$ & 109.1 \\
\hline $\mathrm{C} 6-\mathrm{C} 5-\mathrm{H} 5$ & 109.5 & $\mathrm{~N} 1-\mathrm{C} 17-\mathrm{H} 17 \mathrm{~B}$ & 109.1 \\
\hline $\mathrm{C} 9-\mathrm{C} 5-\mathrm{H} 5$ & 109.5 & $\mathrm{C} 15-\mathrm{C} 17-\mathrm{H} 17 \mathrm{~B}$ & 109.1 \\
\hline $\mathrm{C} 5-\mathrm{C} 6-\mathrm{C} 7$ & $109.62(14)$ & $\mathrm{H} 17 \mathrm{~A}-\mathrm{C} 17-\mathrm{H} 17 \mathrm{~B}$ & 107.8 \\
\hline $\mathrm{C} 5-\mathrm{C} 6-\mathrm{H} 6 \mathrm{~A}$ & 109.7 & $\mathrm{~N} 1-\mathrm{C} 18-\mathrm{N} 2$ & $108.88(15)$ \\
\hline $\mathrm{C} 7-\mathrm{C} 6-\mathrm{H} 6 \mathrm{~A}$ & 109.7 & $\mathrm{~N} 1-\mathrm{C} 18-\mathrm{H} 18$ & 125.6 \\
\hline $\mathrm{C} 5-\mathrm{C} 6-\mathrm{H} 6 \mathrm{AB}$ & 109.7 & $\mathrm{~N} 2-\mathrm{C} 18-\mathrm{H} 18$ & 125.6 \\
\hline C7-C6-H6AB & 109.7 & $\mathrm{C} 20-\mathrm{C} 19-\mathrm{N} 1$ & $106.98(16)$ \\
\hline
\end{tabular}




\begin{tabular}{llll}
\hline H6A-C6-H6AB & 108.2 & $\mathrm{C} 20-\mathrm{C} 19-\mathrm{H} 19$ & 126.5 \\
C10-C7-C8 & $109.27(15)$ & $\mathrm{N} 1-\mathrm{C} 19-\mathrm{H} 19$ & 126.5 \\
C10-C7-C6 & $109.59(16)$ & $\mathrm{C} 19-\mathrm{C} 20-\mathrm{N} 2$ & $107.04(15)$ \\
C8-C7-C6 & $109.09(15)$ & $\mathrm{C} 19-\mathrm{C} 20-\mathrm{H} 20$ & 126.5 \\
C10-C7-H7 & 109.6 & $\mathrm{~N} 2-\mathrm{C} 20-\mathrm{H} 20$ & 126.5 \\
C8-C7-H7 & 109.6 & $\mathrm{~N} 2-\mathrm{C} 21-\mathrm{H} 21 \mathrm{~A}$ & 109.5 \\
C6-C7-H7 & 109.6 & $\mathrm{~N} 2-\mathrm{C} 21-\mathrm{H} 21 \mathrm{~B}$ & 109.5 \\
C7-C8-C1 & $111.00(15)$ & $\mathrm{H} 21 \mathrm{~A}-\mathrm{C} 21-\mathrm{H} 21 \mathrm{~B}$ & 109.5 \\
C7-C8-H8A & 109.4 & $\mathrm{~N} 2-\mathrm{C} 21-\mathrm{H} 21 \mathrm{C}$ & 109.5 \\
C1-C8-H8A & 109.4 & $\mathrm{H} 21 \mathrm{~A}-\mathrm{C} 21-\mathrm{H} 21 \mathrm{C}$ & 109.5 \\
C7-C8-H8AB & 109.4 & $\mathrm{H} 21 \mathrm{~B}-\mathrm{C} 21-\mathrm{H} 21 \mathrm{C}$ & 109.5 \\
C1-C8-H8AB & 109.4 & & \\
\hline
\end{tabular}

Table S6: Fractional atomic coordinates and isotropic or equivalent isotropic displacement parameters $\left(\AA^{2}\right)$ for compound $\mathbf{5 b}$.

\begin{tabular}{lllll}
\hline & $\mathrm{x}$ & $\mathrm{y}$ & $\mathrm{z}$ & $\mathrm{U}_{\text {iso }} * / \mathrm{U}_{\mathrm{eq}}$ \\
\hline $\mathrm{C} 1$ & $0.34014(9)$ & $0.6146(2)$ & $0.54769(13)$ & $0.0168(3)$ \\
$\mathrm{C} 2$ & 0.293537 & 0.586546 & 0.673622 & $0.023^{*}$ \\
$\mathrm{H} 2 \mathrm{AB}$ & 0.242743 & 0.705438 & 0.590514 & $0.023^{*}$ \\
$\mathrm{C} 3$ & $0.33882(10)$ & $0.8200(2)$ & $0.68461(14)$ & $0.0221(4)$ \\
$\mathrm{H} 3$ & 0.309669 & 0.856909 & 0.734916 & $0.027^{*}$ \\
$\mathrm{C} 4$ & $0.42198(10)$ & $0.7703(2)$ & $0.74040(14)$ & $0.0237(4)$ \\
H4A & 0.449269 & 0.862846 & 0.779279 & $0.028^{*}$ \\
H4B & 0.419672 & 0.682350 & 0.788615 & $0.028^{*}$ \\
$\mathrm{C} 5$ & $0.46673(10)$ & $0.7140(2)$ & $0.66377(14)$ & $0.0228(4)$ \\
H5 & 0.520856 & 0.681978 & 0.700196 & $0.027^{*}$ \\
C6 & $0.47056(10)$ & $0.8500(2)$ & $0.58908(15)$ & $0.0267(4)$ \\
H6A & 0.499318 & 0.812957 & 0.539277 & $0.032^{*}$ \\
H6AB & 0.498791 & 0.943164 & 0.626060 & $0.032^{*}$ \\
C7 & $0.38735(11)$ & $0.9006(2)$ & $0.53375(15)$ & $0.0260(4)$ \\
H7 & 0.389843 & 0.990008 & 0.485473 & $0.031^{*}$ \\
C8 & $0.34486(10)$ & $0.7555(2)$ & $0.47516(14)$ & $0.0226(4)$ \\
H8A & 0.372994 & 0.719866 & 0.424374 & $0.027 *$ \\
H8AB & 0.291469 & 0.787834 & 0.438691 & $0.027^{*}$ \\
C9 & $0.42430(10)$ & $0.5684(2)$ & $0.60549(14)$ & $0.0193(4)$ \\
\hline & & & & \\
\hline
\end{tabular}




\begin{tabular}{|c|c|c|c|c|}
\hline H9A & 0.422235 & 0.480124 & 0.653567 & $0.023 *$ \\
\hline H9AB & 0.453636 & 0.529527 & 0.556777 & $0.023 *$ \\
\hline $\mathrm{C} 10$ & $0.34289(11)$ & $0.9572(2)$ & $0.61114(16)$ & $0.0267(4)$ \\
\hline $\mathrm{H} 10 \mathrm{~A}$ & 0.369830 & 1.050840 & 0.649076 & $0.032 *$ \\
\hline $\mathrm{H} 10 \mathrm{~B}$ & 0.289458 & 0.990730 & 0.575679 & $0.032 *$ \\
\hline $\mathrm{C} 11$ & $0.30035(9)$ & $0.4711(2)$ & $0.48580(13)$ & $0.0170(3)$ \\
\hline $\mathrm{C} 12$ & $0.33626(10)$ & $0.3938(2)$ & $0.41764(14)$ & $0.0218(4)$ \\
\hline $\mathrm{H} 12$ & 0.385691 & 0.430800 & 0.411131 & $0.026^{*}$ \\
\hline $\mathrm{C} 13$ & $0.30141(10)$ & $0.2645(2)$ & $0.35944(14)$ & $0.0242(4)$ \\
\hline H13 & 0.327352 & 0.213482 & 0.314413 & $0.029 *$ \\
\hline C14 & $0.22854(10)$ & $0.2093(2)$ & $0.36675(13)$ & $0.0207(4)$ \\
\hline $\mathrm{H} 14$ & 0.204748 & 0.120399 & 0.327191 & $0.025^{*}$ \\
\hline $\mathrm{C} 15$ & $0.19085(9)$ & $0.2858(2)$ & $0.43273(13)$ & $0.0177(3)$ \\
\hline $\mathrm{C} 16$ & $0.22680(10)$ & $0.4145(2)$ & $0.49174(13)$ & $0.0170(3)$ \\
\hline H16 & 0.200870 & 0.464909 & 0.537001 & $0.020 *$ \\
\hline $\mathrm{C} 17$ & $0.10976(9)$ & $0.2341(2)$ & $0.43836(13)$ & $0.0184(3)$ \\
\hline H17A & 0.085356 & 0.321746 & 0.469176 & $0.022 *$ \\
\hline H17B & 0.077419 & 0.216141 & 0.368902 & $0.022 *$ \\
\hline $\mathrm{C} 18$ & $0.13398(9)$ & $0.0767(2)$ & $0.59864(14)$ & $0.0180(4)$ \\
\hline $\mathrm{H} 18$ & 0.155050 & 0.163152 & 0.642466 & $0.022 *$ \\
\hline C19 & $0.08312(10)$ & $-0.06401(19)$ & $0.46139(14)$ & $0.0191(4)$ \\
\hline H19 & 0.062675 & -0.091800 & 0.392336 & $0.023 *$ \\
\hline $\mathrm{C} 20$ & $0.09085(9)$ & $-0.1630(2)$ & $0.54210(14)$ & $0.0198(4)$ \\
\hline $\mathrm{H} 20$ & 0.076853 & -0.273475 & 0.540458 & $0.024 *$ \\
\hline $\mathrm{C} 21$ & $0.14029(10)$ & $-0.1286(2)$ & $0.73309(14)$ & $0.0240(4)$ \\
\hline $\mathrm{H} 21 \mathrm{~A}$ & 0.091853 & -0.132742 & 0.756032 & $0.036^{*}$ \\
\hline $\mathrm{H} 21 \mathrm{~B}$ & 0.176973 & -0.054232 & 0.776083 & $0.036^{*}$ \\
\hline $\mathrm{H} 21 \mathrm{C}$ & 0.163511 & -0.236258 & 0.737516 & $0.036^{*}$ \\
\hline
\end{tabular}

Table S7: Atomic displacement parameters $\left(\AA^{2}\right)$ for compound $\mathbf{5 b}$.

\begin{tabular}{lllllll}
\hline & $\mathrm{U}^{11}$ & $\mathrm{U}^{22}$ & $\mathrm{U}^{33}$ & $\mathrm{U}^{23}$ & $\mathrm{U}^{13}$ & $\mathrm{U}^{12}$ \\
\hline $\mathrm{I} 1$ & $0.02427(8)$ & $0.01808(7)$ & $0.01771(8)$ & $-0.00251(4)$ & $0.00477(5)$ & $-0.00283(4)$ \\
$\mathrm{N} 1$ & $0.0135(6)$ & $0.0162(7)$ & $0.0190(8)$ & $-0.0004(5)$ & $0.0036(6)$ & $0.0001(5)$ \\
$\mathrm{N} 2$ & $0.0160(7)$ & $0.0183(7)$ & $0.0205(8)$ & $0.0002(5)$ & $0.0064(6)$ & $0.0009(5)$ \\
$\mathrm{C} 1$ & $0.0142(8)$ & $0.0190(8)$ & $0.0169(9)$ & $-0.0005(7)$ & $0.0034(6)$ & $-0.0004(6)$ \\
\hline
\end{tabular}




\begin{tabular}{lllllll}
\hline C2 & $0.0147(8)$ & $0.0232(9)$ & $0.0199(9)$ & $-0.0034(7)$ & $0.0052(6)$ & $-0.0004(6)$ \\
C3 & $0.0172(8)$ & $0.0265(9)$ & $0.0234(10)$ & $-0.0074(7)$ & $0.0061(7)$ & $0.0000(7)$ \\
C4 & $0.0203(9)$ & $0.0285(10)$ & $0.0212(9)$ & $-0.0054(7)$ & $0.0028(7)$ & $-0.0023(7)$ \\
C5 & $0.0140(8)$ & $0.0291(10)$ & $0.0238(10)$ & $-0.0042(8)$ & $0.0019(7)$ & $-0.0005(7)$ \\
C6 & $0.0225(9)$ & $0.0281(10)$ & $0.0312(11)$ & $-0.0058(8)$ & $0.0100(8)$ & $-0.0081(7)$ \\
C7 & $0.0288(10)$ & $0.0213(9)$ & $0.0278(10)$ & $0.0031(8)$ & $0.0068(8)$ & $-0.0044(8)$ \\
C8 & $0.0236(9)$ & $0.0243(9)$ & $0.0195(9)$ & $0.0023(7)$ & $0.0045(7)$ & $-0.0019(7)$ \\
C9 & $0.0148(8)$ & $0.0231(9)$ & $0.0201(9)$ & $-0.0012(7)$ & $0.0045(7)$ & $0.0016(6)$ \\
C10 & $0.0234(9)$ & $0.0207(9)$ & $0.0342(12)$ & $-0.0051(7)$ & $0.0035(8)$ & $0.0003(7)$ \\
C11 & $0.0164(8)$ & $0.0184(8)$ & $0.0156(9)$ & $0.0014(6)$ & $0.0029(7)$ & $0.0007(6)$ \\
C12 & $0.0172(8)$ & $0.0261(9)$ & $0.0235(10)$ & $-0.0017(7)$ & $0.0075(7)$ & $-0.0010(7)$ \\
C13 & $0.0236(9)$ & $0.0272(10)$ & $0.0236(10)$ & $-0.0044(7)$ & $0.0094(7)$ & $0.0023(7)$ \\
C14 & $0.0232(9)$ & $0.0185(8)$ & $0.0195(9)$ & $-0.0028(7)$ & $0.0033(7)$ & $-0.0001(7)$ \\
C15 & $0.0171(8)$ & $0.0172(8)$ & $0.0177(9)$ & $0.0039(6)$ & $0.0023(6)$ & $0.0003(6)$ \\
C16 & $0.0171(8)$ & $0.0189(8)$ & $0.0154(9)$ & $0.0010(6)$ & $0.0048(7)$ & $0.0016(6)$ \\
C17 & $0.0178(8)$ & $0.0166(8)$ & $0.0200(9)$ & $0.0018(6)$ & $0.0032(7)$ & $-0.0003(6)$ \\
C18 & $0.0158(8)$ & $0.0192(9)$ & $0.0191(9)$ & $-0.0018(6)$ & $0.0042(7)$ & $0.0005(6)$ \\
C19 & $0.0186(8)$ & $0.0171(9)$ & $0.0211(10)$ & $-0.0030(6)$ & $0.0042(7)$ & $-0.0025(6)$ \\
C20 & $0.0181(8)$ & $0.0173(8)$ & $0.0244(9)$ & $-0.0021(7)$ & $0.0059(7)$ & $-0.0015(6)$ \\
C21 & $0.0259(9)$ & $0.0279(10)$ & $0.0193(9)$ & $0.0033(7)$ & $0.0079(7)$ & $0.0013(7)$ \\
\hline & & & & & & \\
\hline
\end{tabular}




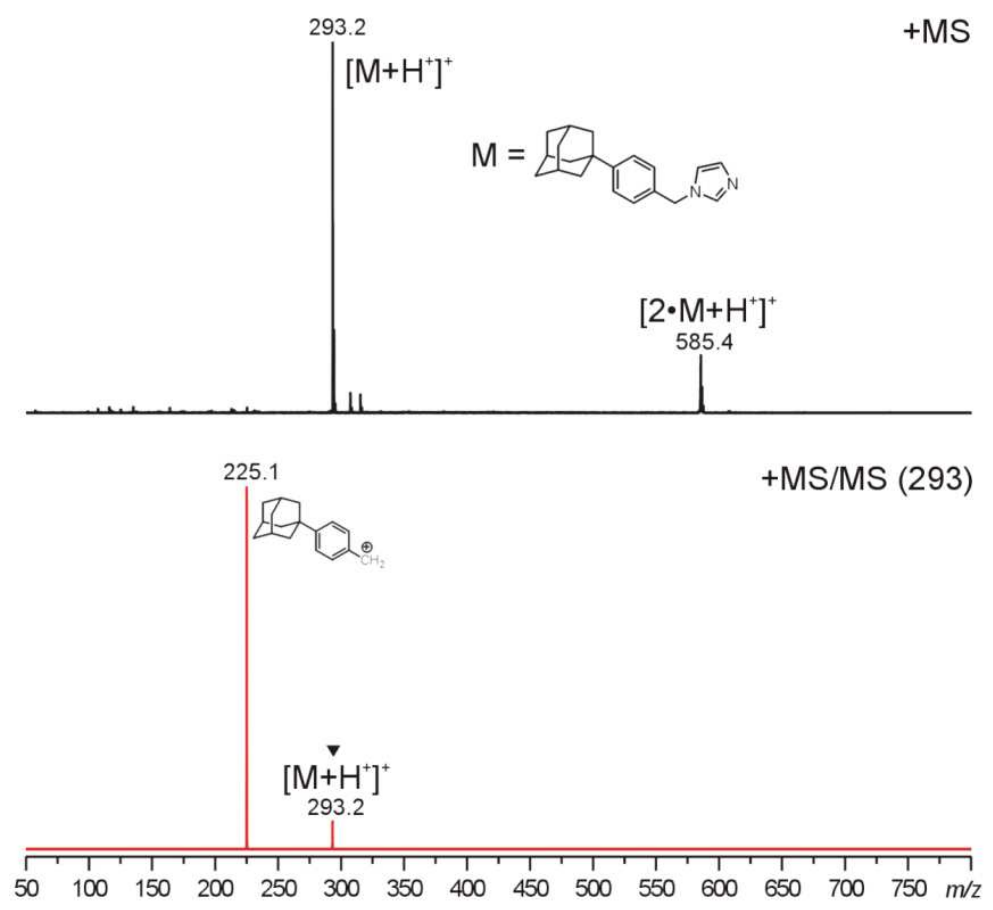

Figure S43 The positive-ion first-order ESI-MS (black line) and MS/MS (red line) of a $\mathrm{MeOH}: \mathrm{H}_{2} \mathrm{O}$ $(1: 1, \mathrm{v}: \mathrm{v})$ solution of compound $\mathbf{4 a}$. The assignments for observed signals are shown in the brackets. The fragmented ion in MS/MS spectra is marked with a downward-facing triangle.

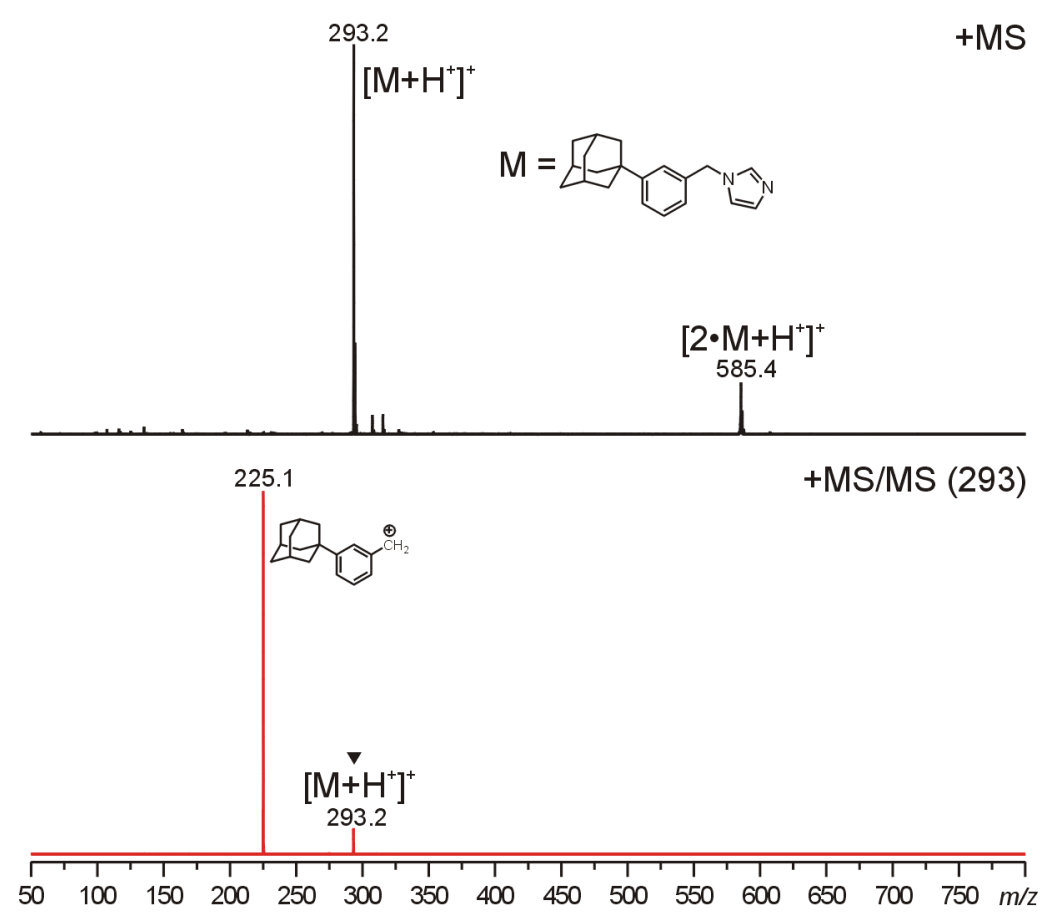

Figure S44 The positive-ion first-order ESI-MS (black line) and MS/MS (red line) of a $\mathrm{MeOH}: \mathrm{H}_{2} \mathrm{O}$ $(1: 1, \mathrm{v}: \mathrm{v})$ solution of compound $\mathbf{4 b}$. The assignments for observed signals are shown in the brackets. The fragmented ion in MS/MS spectra is marked with a downward-facing triangle. 


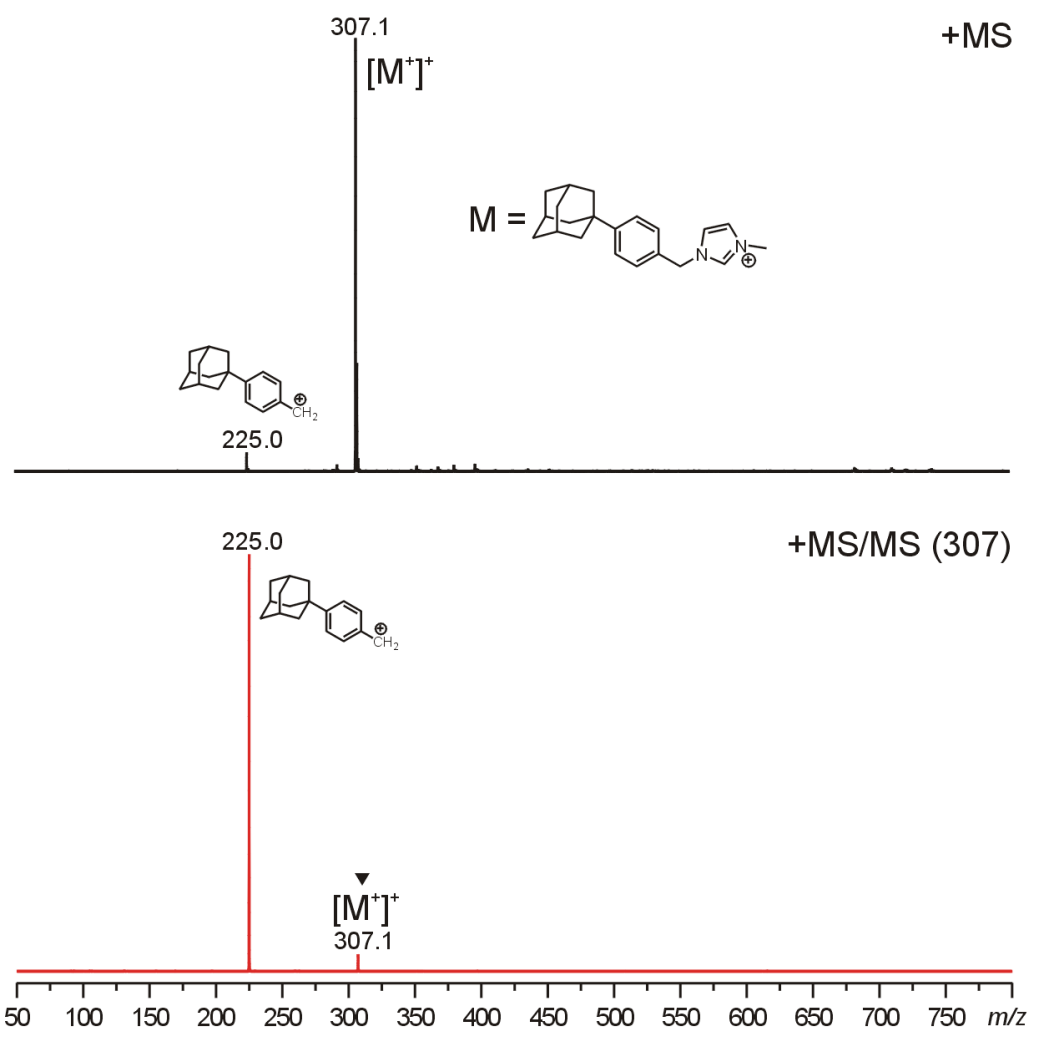

Figure S45 The positive-ion first-order ESI-MS (black line) and MS/MS (red line) of a $\mathrm{MeOH}: \mathrm{H}_{2} \mathrm{O}$ $(1: 1, \mathrm{v}: \mathrm{v})$ solution of compound 5a. The assignments for observed signals are shown in the brackets. The fragmented ion in MS/MS spectra is marked with a downward-facing triangle.

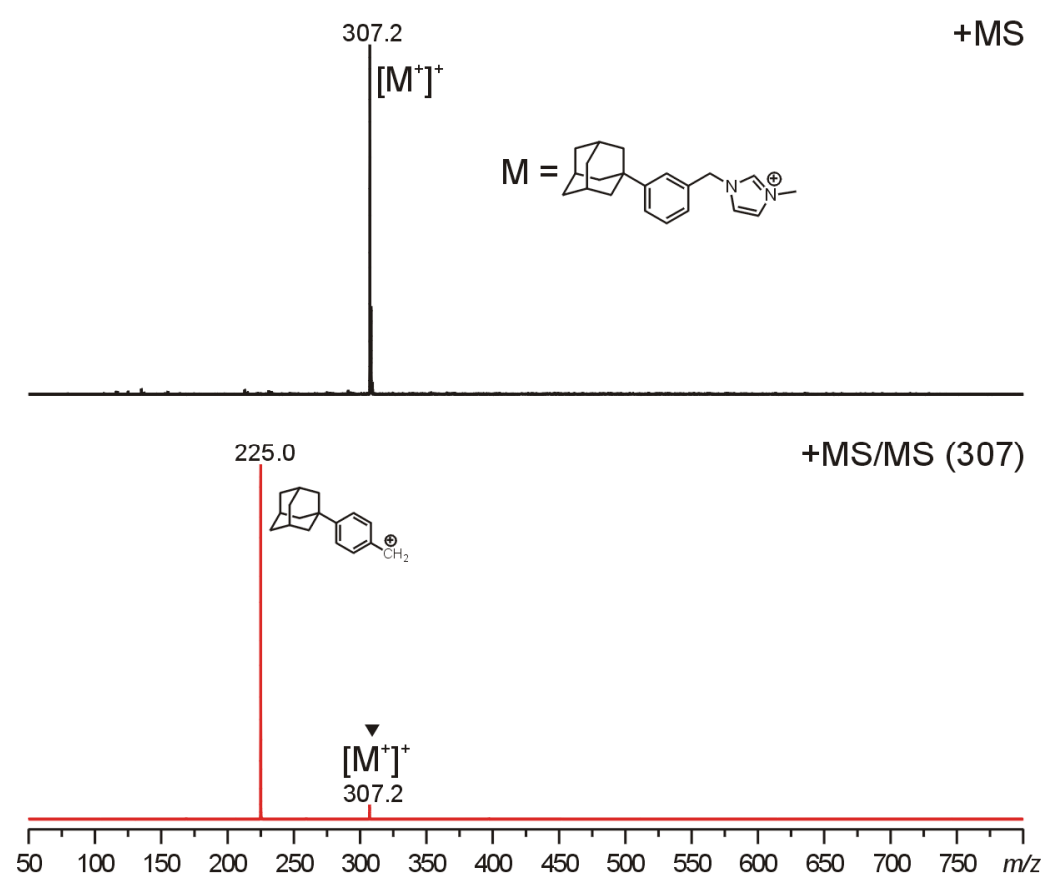

Figure S46 The positive-ion first-order ESI-MS (black line) and MS/MS (red line) of a $\mathrm{MeOH}: \mathrm{H}_{2} \mathrm{O}$ $(1: 1, \mathrm{v}: \mathrm{v})$ solution of compound $\mathbf{5 b}$. The assignments for observed signals are shown in the brackets. The fragmented ion in MS/MS spectra is marked with a downward-facing triangle. 


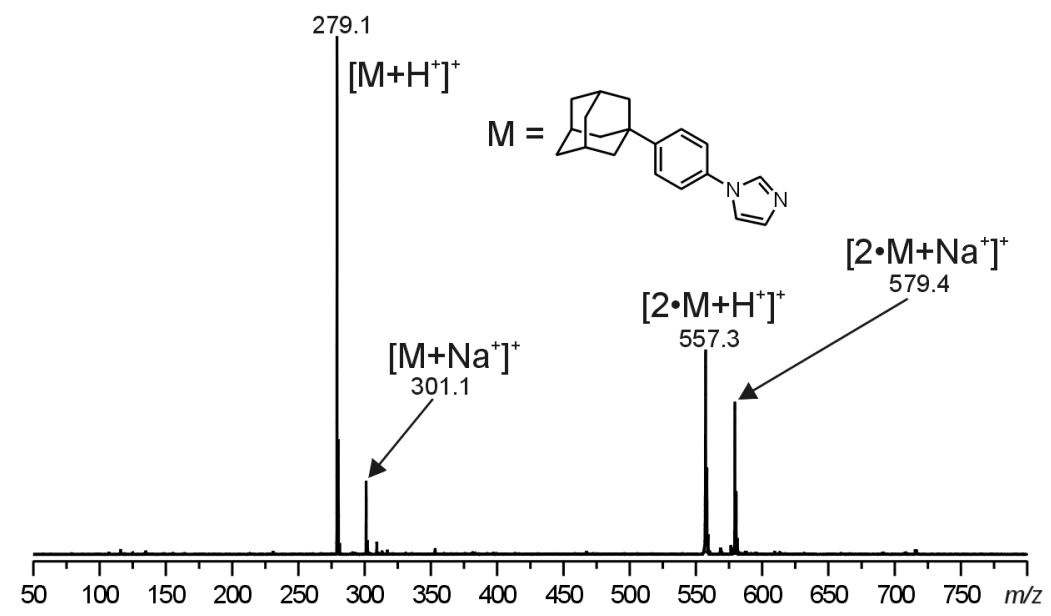

Figure S47 The positive-ion first-order ESI-MS of a MeOH: $\mathrm{H}_{2} \mathrm{O}(1: 1, \mathrm{v}: \mathrm{v})$ solution of compound 9. The assignments for observed signals are shown in the brackets.

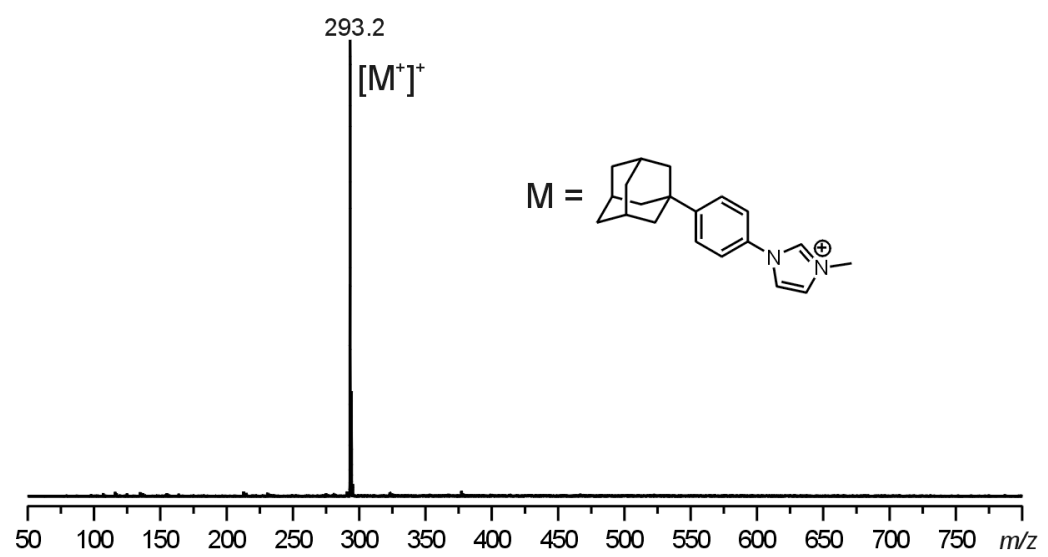

Figure S48 The positive-ion first-order ESI-MS of a $\mathrm{MeOH}: \mathrm{H}_{2} \mathrm{O}(1: 1, \mathrm{v}: \mathrm{v})$ solution of compound $\mathbf{1 0}$. The assignment for observed signals is shown in the brackets.

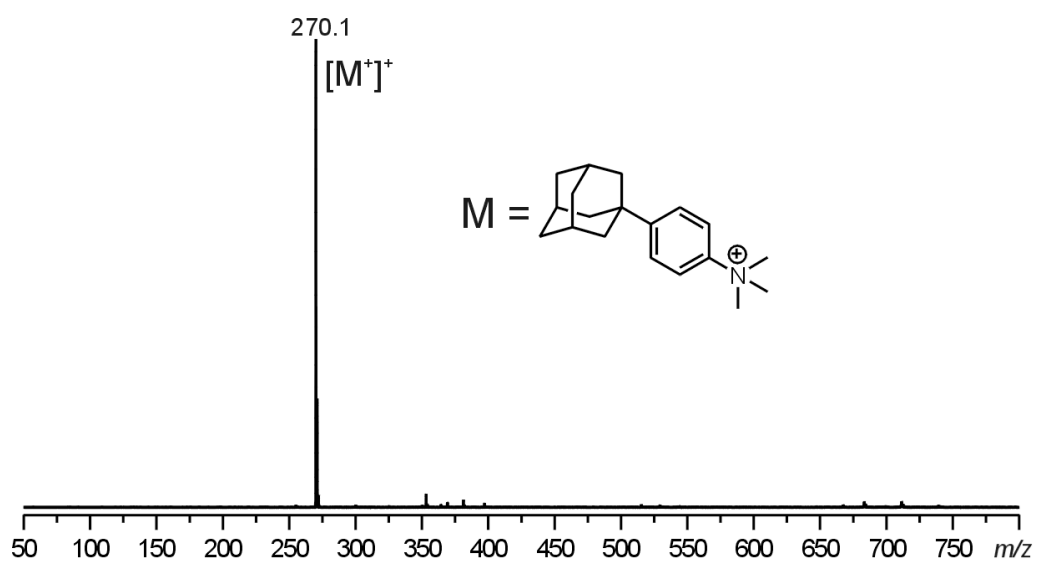

Figure S49 The positive-ion first-order ESI-MS of a $\mathrm{MeOH}: \mathrm{H}_{2} \mathrm{O}$ (1:1, v:v) solution of compound 11. The assignment for observed signals is shown in the brackets. 


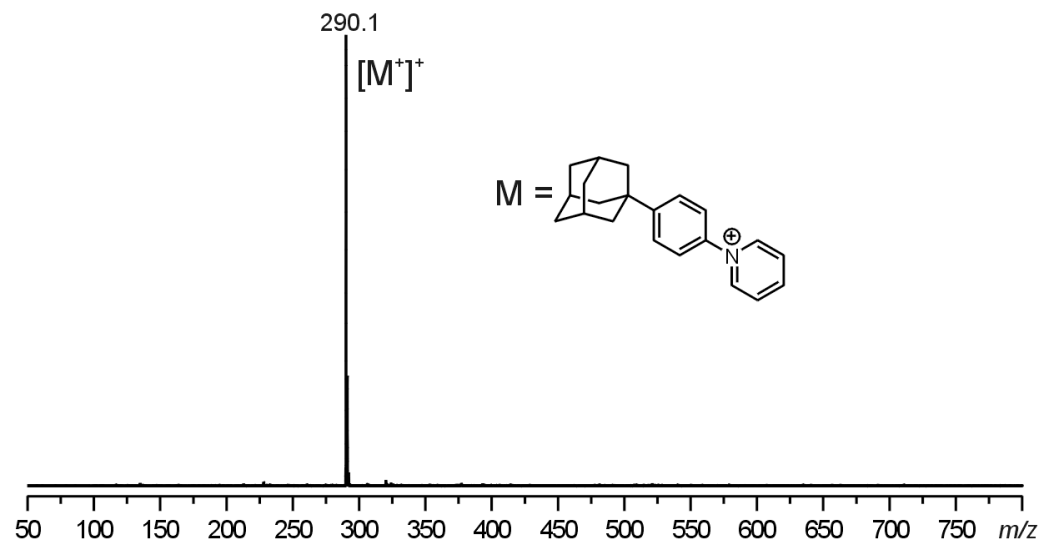

Figure S50 The positive-ion first-order ESI-MS of a MeOH: $\mathrm{H}_{2} \mathrm{O}(1: 1, \mathrm{v}: \mathrm{v})$ solution of compound 12. The assignment for observed signals is shown in the brackets.

S38 


\section{ESI-MS spectra of complexes}

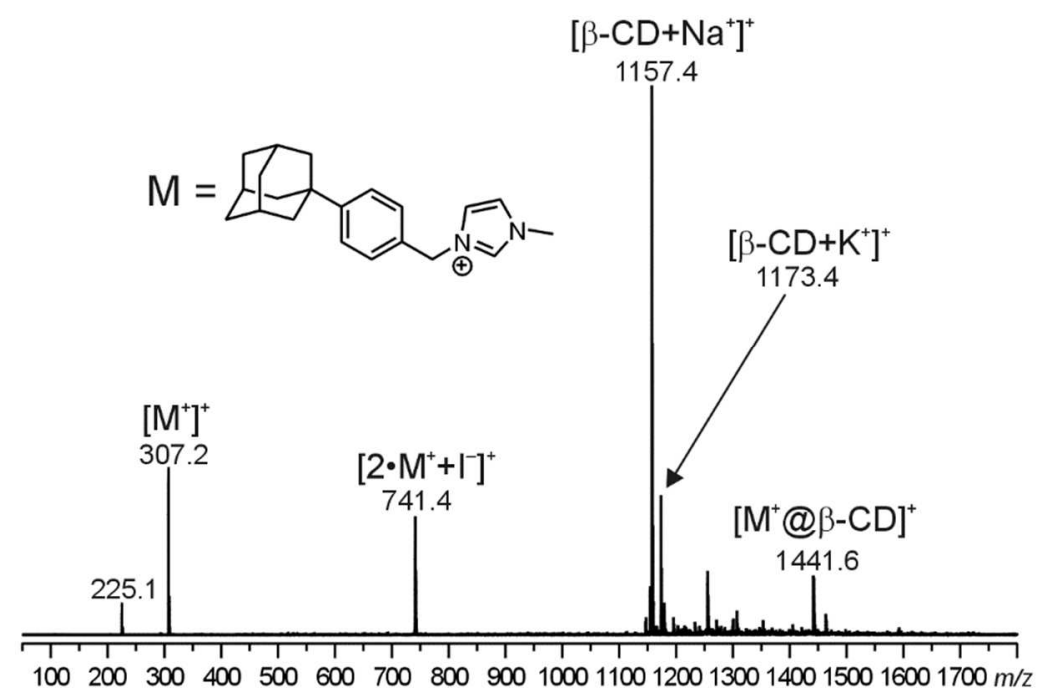

Figure S51 The positive-ion first-order ESI-MS of an aqueous solution of 5a @ $\beta$-CD in molar ratio 1:1. The assignments for observed signals are shown in the brackets.

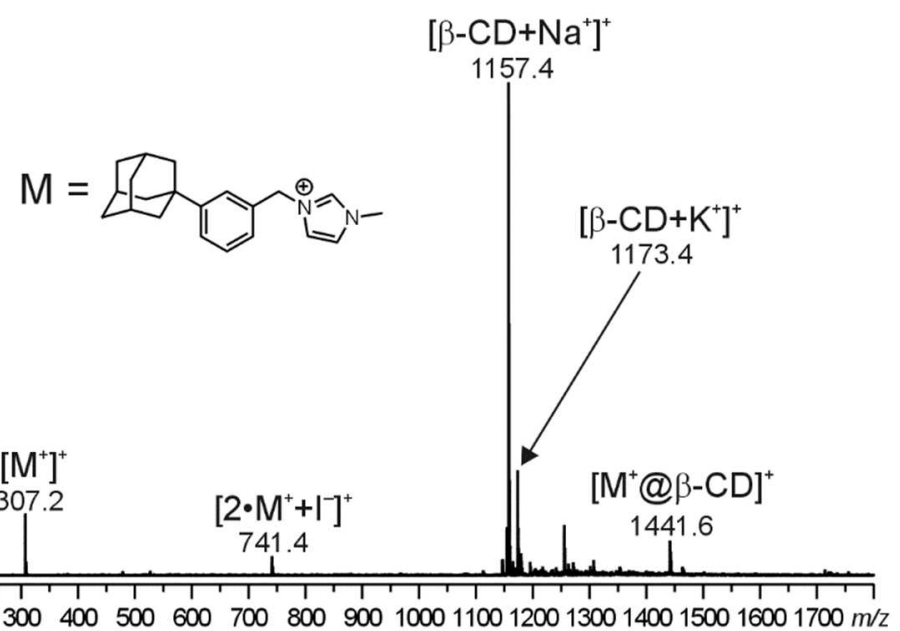

$10020030040050060070080090010001100120013001400150016001700 \mathrm{~m} / \mathrm{z}$

Figure S52 The positive-ion first-order ESI-MS of an aqueous solution of $\mathbf{5 b} @ \beta$-CD in molar ratio 1:1. The assignments for observed signals are shown in the brackets. 


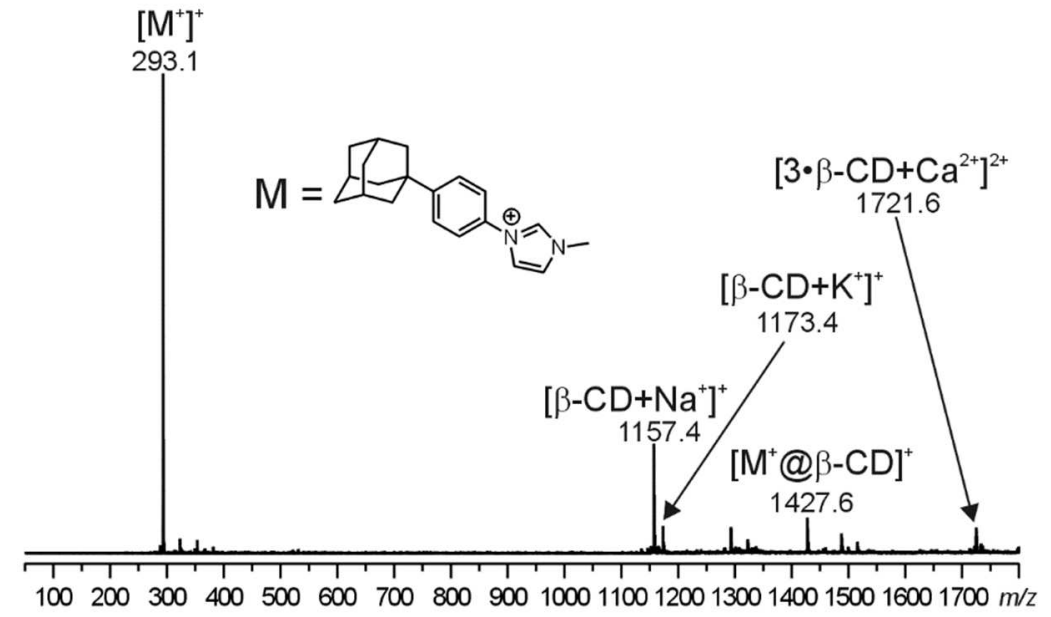

Figure S53 The positive-ion first-order ESI-MS of an aqueous solution of 10@ $\beta$-CD in molar ratio 1:1. The assignments for observed signals are shown in the brackets.

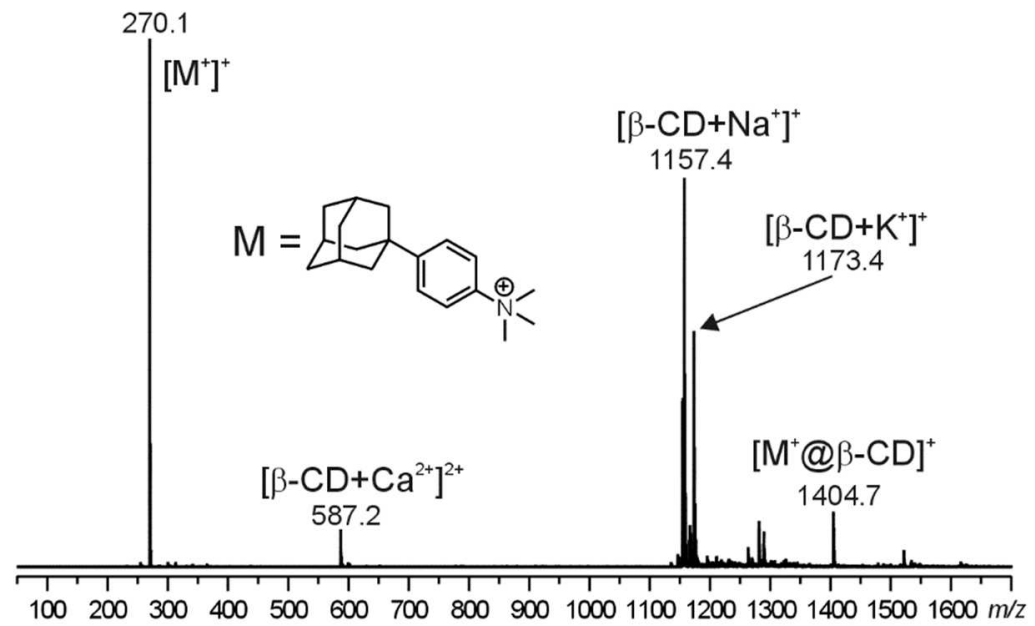

Figure S54 The positive-ion first-order ESI-MS of an aqueous solution of 11@ $\beta$-CD in molar ratio 1:1. The assignments for observed signals are shown in the brackets.

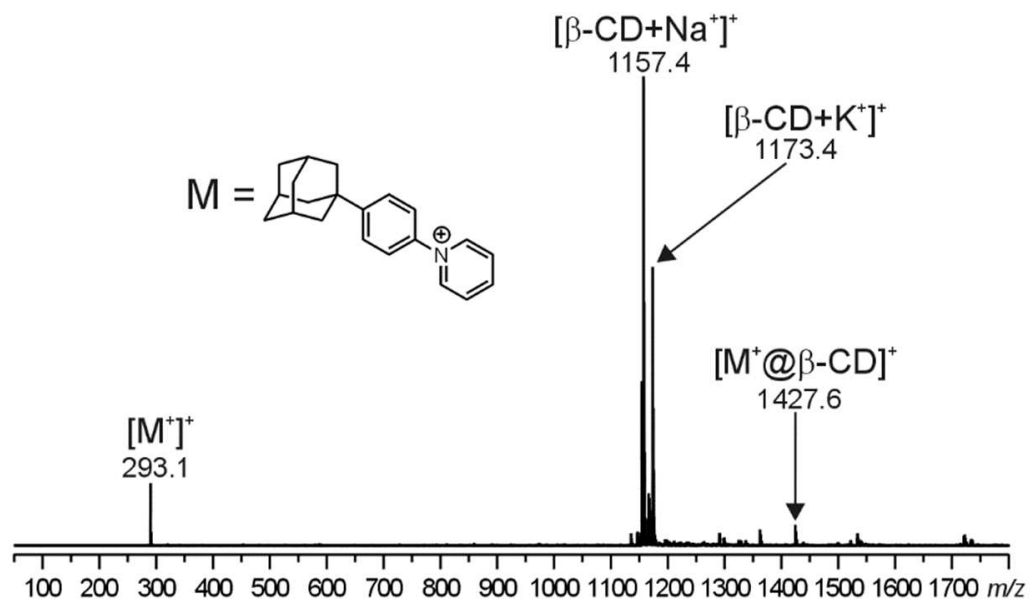

Figure S55 The positive-ion first-order ESI-MS of an aqueous solution of 12@ $\beta$-CD in molar ratio 1:3. The assignments for observed signals are shown in the brackets. 

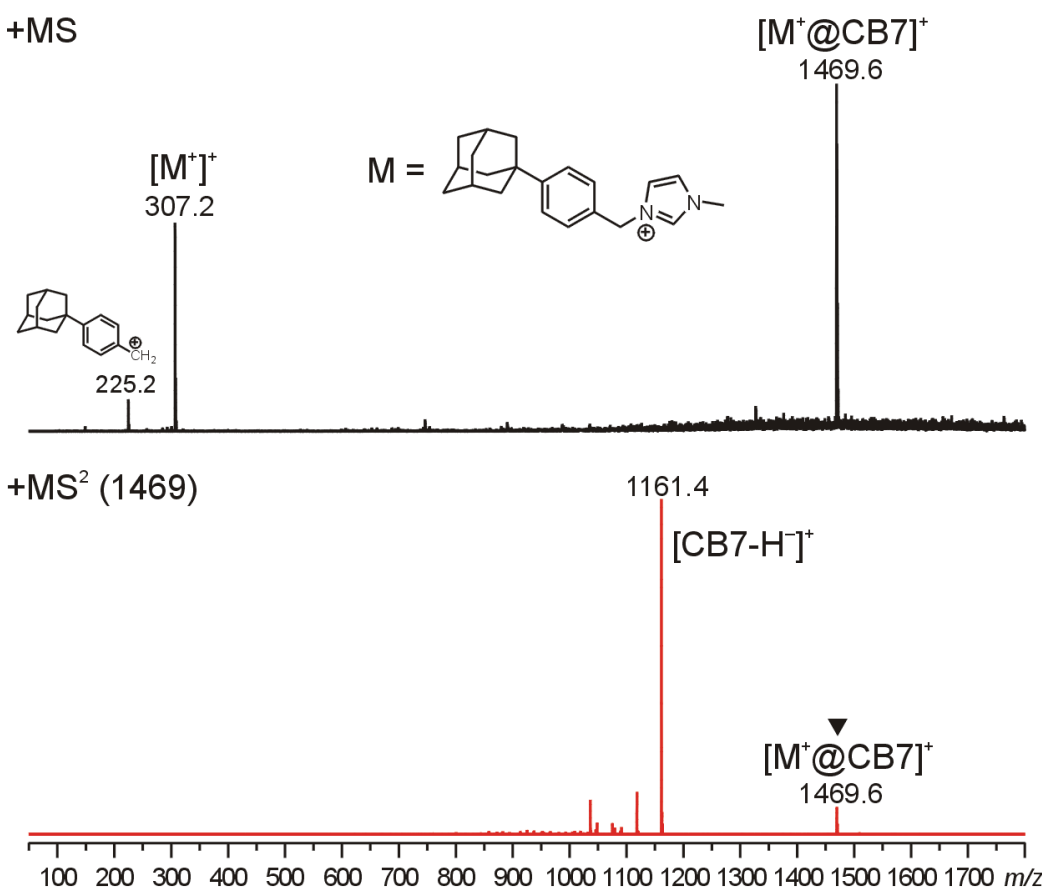

Figure S56 The positive-ion first-order ESI-MS (black line) and MS/MS (red line) of an aqueous solution of 5a@CB7 in molar ratio 1:1. The assignments for observed signals are shown in the brackets. The fragmented ion in MS/MS spectra is marked with a downward-facing triangle.

+ MS

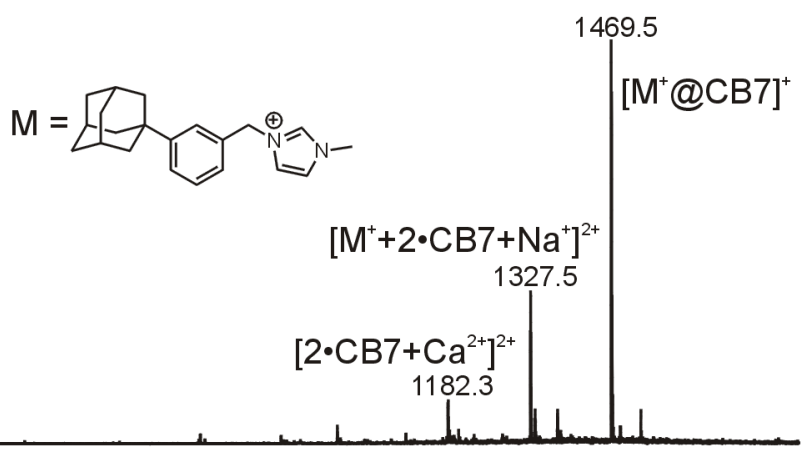

$+\mathrm{MS}^{2}(1469)$

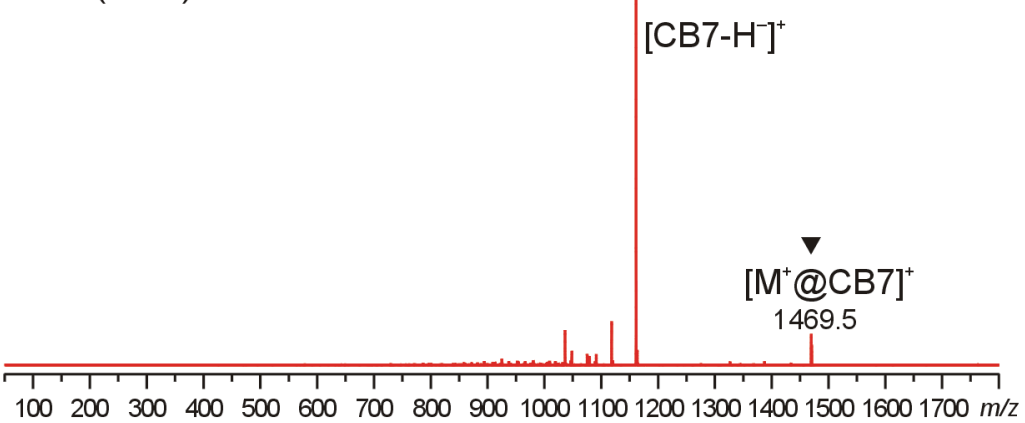

Figure S57 The positive-ion first-order ESI-MS (black line) and MS/MS (red line) of an aqueous solution of 5b@CB7 in molar ratio 1:1. The assignments for observed signals are shown in the brackets. The fragmented ion in MS/MS spectra is marked with a downward-facing triangle. 


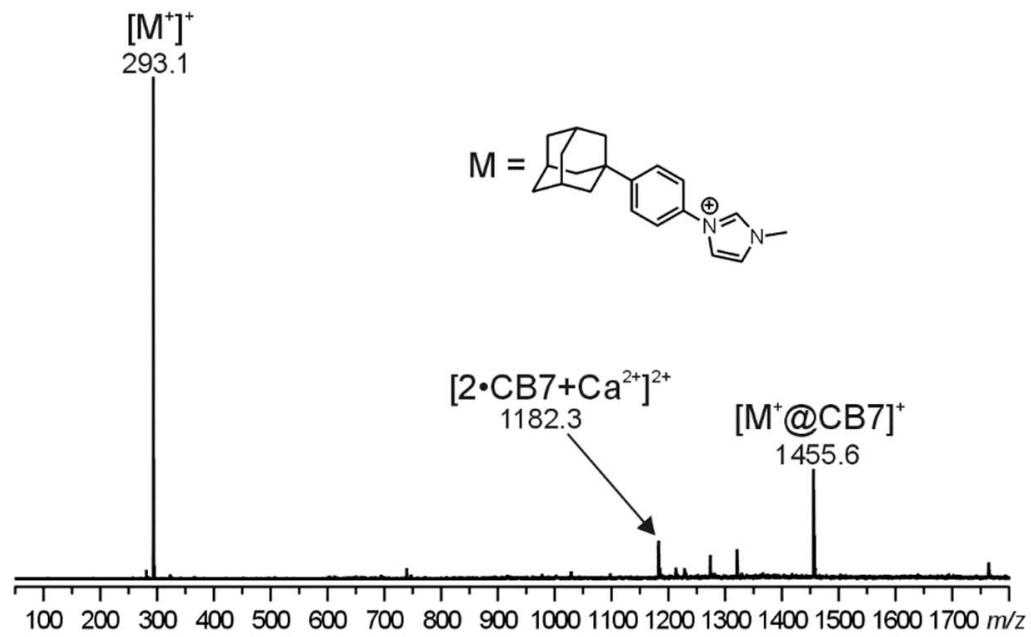

Figure S58 The positive-ion first-order ESI-MS of an aqueous solution of 10@CB7 in molar ratio 1:1. The assignments for observed signals are shown in the brackets.

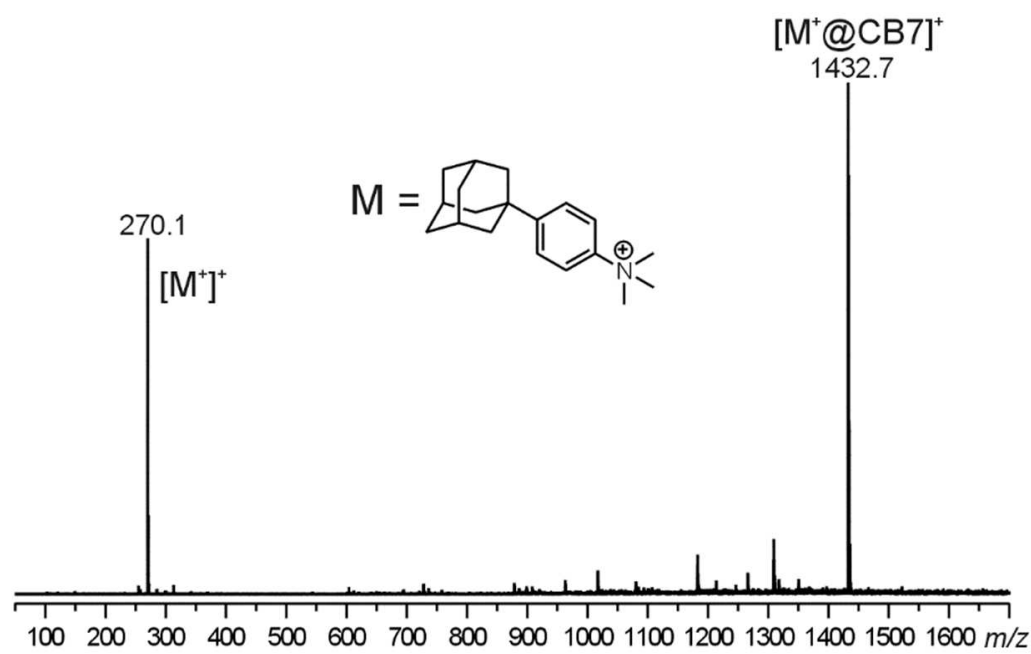

Figure S59 The positive-ion first-order ESI-MS of an aqueous solution of 11@CB7 in molar ratio 1:1. The assignments for observed signals are shown in the brackets. 


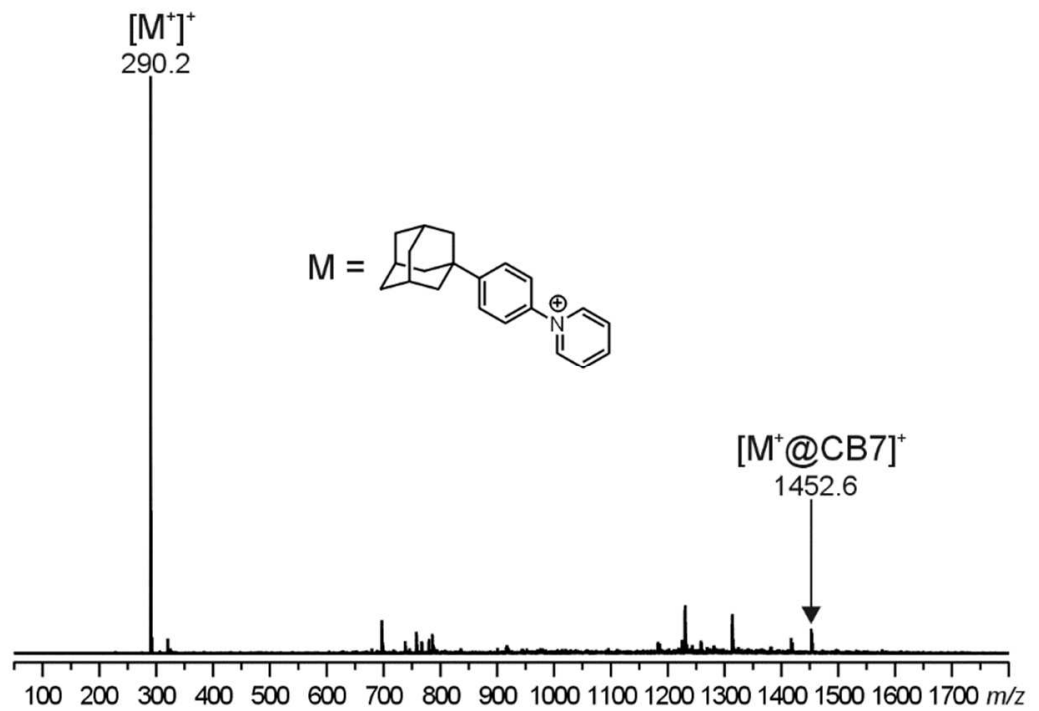

Figure S60 The positive-ion first-order ESI-MS of an aqueous solution of 12@CB7 in molar ratio 1:1. The assignments for observed signals are shown in the brackets.
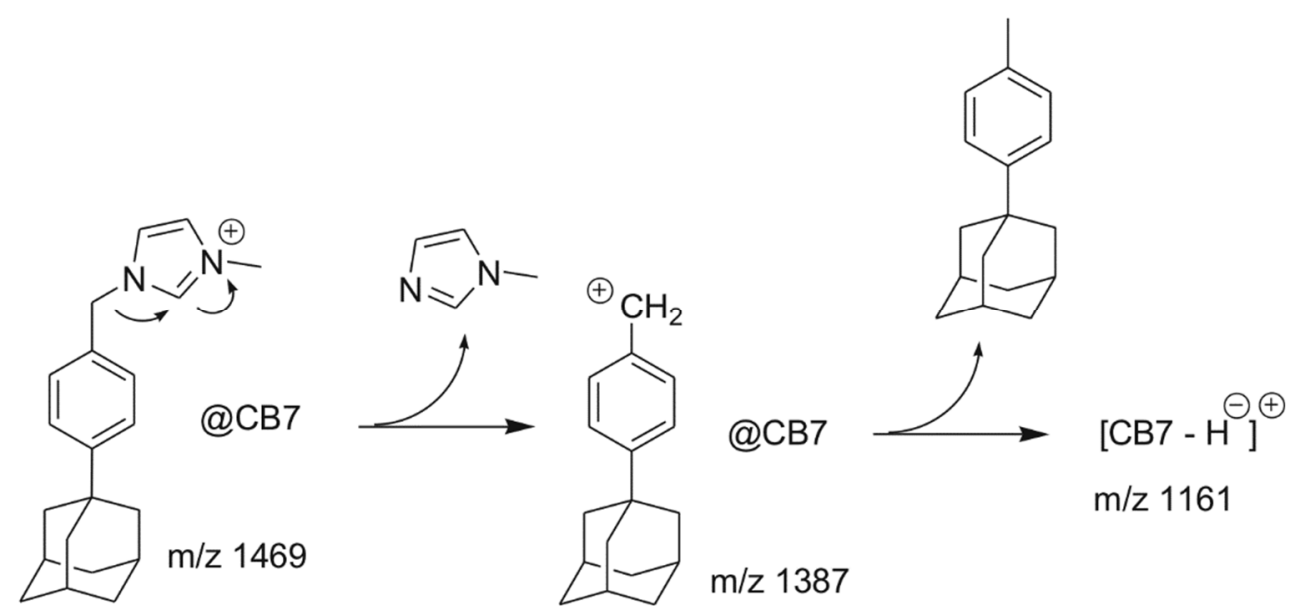

Scheme S1 Suggested fragmentation of the complex 5a@CB7. 


\section{Computation Details}

\section{$11 @ \beta-C D$ Complex Preparation}

Structure of the guest 11 was optimised at TPSSh-D3BJ/def2-TZVPP level of theory ${ }^{3-6}$ employing RI and RIJCOSX approximations and def $2 / \mathrm{J}$ auxiliary basis set ${ }^{7}$ in Orca. ${ }^{8}$ Electrostatic potential (ESP) was calculated on the optimised structure at HF/6-31G* level of theory in Gaussian. ${ }^{9}$ Atomic charges were derived from calculated ESP with the RESP method ${ }^{10}$ implemented in Antechamber, ${ }^{11}$ which was also used to assign General Amber Force Field 2 (GAFF2) atom types and parameters. $\beta$-Cyclodextrin ( $\beta$ CD) was described by a GLYCAM06 force field. ${ }^{12}$ The structure of the supramolecular 11@ $\beta$-CD complex was formed by placing 11 into the $\beta-C D$ cavity. The complex was then solvated with TIP3P ${ }^{13}$ water model using a solvate package ${ }^{14}$ with a $28 \AA$ thick water shell, which was later transformed into a truncated octahedral box. Sodium and chloride ions ${ }^{15}$ were added to neutralise the net charge of $11 @ \beta$ $\mathrm{CD}$ and to increase the ionic strength of the solution $\left(c_{\mathrm{NaCl}} \approx 0.14 \mathrm{M}\right)$ to improve the sampling of ions around the solute. Because $\mathbf{1 1}$ can be positioned in two orientations inside the $\beta$-CD cavity, the other orientation was prepared by rotating encapsulated 11 by $180^{\circ}$. Both orientations will be referred to as NP and NS according to the nitrogen atom of $\mathbf{1 1}$ pointing in the direction of the primary or secondary portal, respectively.

\section{Unbiased Molecular Dynamics}

All molecular dynamics (MD) simulations were done with the AMBER 16 software package ${ }^{16}$ employing the periodic boundary conditions with vdW interactions considered up to $9 \AA$. Electrostatic interactions were treated with $\mathrm{PME}^{17}$ with a direct summation truncated at the same cut-off as used for $\mathrm{vdW}$ interactions. The system was equilibrated in three stages prior to further molecular dynamics simulations. First, the geometry of the systems was optimised. In the next stage, the system was gradually heated from 1 to $300 \mathrm{~K}$ during $100 \mathrm{ps}$ long MD simulation employing an integration step of 1 fs. The systems were kept at a constant volume and temperature, which was controlled by stochastic Langevin thermostat ${ }^{18}$ (the collision frequency $\gamma$ was set to $5.0 \mathrm{ps}^{-1}$ ). Bonds containing hydrogen atoms were kept fixed by the SHAKE algorithm. ${ }^{19}$ The third stage of equilibration was 500 ps long (300 K, $\gamma=2.0 \mathrm{ps}^{-1}$ ). The aim of this stage was to equilibrate the density of the system. Thus in addition to the thermostat, a barostat was applied and set to $100 \mathrm{kPa}$ (the pressure relaxation time $\tau_{\mathrm{p}}$ was set to $1.2 \mathrm{ps}$ ). Production dynamics were performed on graphics cards ${ }^{20}$ and had similar settings as the last stage of equilibration. However, cut off for vdW interactions, and the PME method was decreased to $8.0 \AA$, and the integration step was prolonged to $2 \mathrm{fs}$. Langevin thermostat was changed to Berendsen thermostat ${ }^{21}$ (the $\tau_{\mathrm{t}}$ coupling constant was set to $5.0 \mathrm{ps}$ ), which performs better on GPU accelerators and pressure was kept at $100 \mathrm{kPa}\left(\tau_{\mathrm{p}}=6.0 \mathrm{ps}\right)$. Production dynamic provided $1 \mu \mathrm{s}$ long trajectories of NP and NS. 


\section{$\underline{\text { Biased Molecular Dynamics }}$}

Biased MD simulations were performed with the same settings as in the production phase, except thermostat, where the stochastic Langevin thermostat $\left(\gamma=0.1 \mathrm{ps}^{-1}\right)$ was applied to gain better sampling. The Adaptive Biasing Force (ABF) method enhanced by the Multiple Walker Approach (MWA) ${ }^{22}$ implemented in $\mathrm{PMFLib}^{23}$ was used. Initial configurations for 30 walkers were taken from the production phase of NP and NS systems.

$\mathrm{ABF}$ was sampled in the space of two discretised collective variables (Figure S60). Variable $d_{\mathrm{ODIS}}$ was defined as the oriented distance between the centre of mass of non-hydrogen atoms of $\mathbf{1 1}$ and a plane given by the best fit through $\mathrm{C} 1, \mathrm{C} 4$, and $\mathrm{O} 4$ atoms of all $\beta$-CD monosaccharide units. Variable $d_{\text {ODIS }}$ was sampled from -12.0 to $12.0 \AA$ with a bin size of $0.1 \AA$. Harmonic potential with a force constant of $41.38 \mathrm{~kJ} \mathrm{~mol}^{-1} \AA^{-2}$ was applied outside this interval. The second variable $\alpha_{\text {PVANG }}$ was given by an angle between the normal vector of the same $\beta$-CD plane and a vector pointing from the centre of mass of $\mathrm{C} 3, \mathrm{C} 5$, and $\mathrm{C} 7$ adamantane atoms to $\mathrm{N}$ atom (Figure S60). Variable $\alpha_{\mathrm{PVANG}}$ was sampled in the range of 2 to $178^{\circ}$ with a bin size of $2^{\circ}$. To keep the system in a defined state, two restraints were applied. The first was a radial distance $d_{\mathrm{ORAD}}$ measured as the distance between the centre of mass of nonhydrogen atoms of $\mathbf{1 1}$ and the main rotational axis of all $\beta-C D$ heavy atoms. The purpose of $d_{\mathrm{ORAD}}$ wall restraint was to keep $\mathbf{1 1}$ in a cylinder going through the cavity of $\beta$-CD by applying harmonic potential with force constant of $40 \mathrm{~kJ} \cdot \mathrm{mol}^{-1} \cdot \AA^{-2}$ when the value of the distance exceeded $4.0 \AA$. The second one was a root-mean-square deviation of $\beta$-CD structure to the target RMSDT. It prevented a collapse of the $\beta$-CD macrocycle when the complex was dissociated and which otherwise hindered sampling. The targeted structure was prepared as an average structure of $\beta-\mathrm{CD}$ in unbiased MD simulations of the $11 @ \beta-C D$ complex without O6 atoms. Walls of RMSDT restraint were set between 0.0 and $2.0 \AA$ with a force constant of $40 \mathrm{~kJ} \cdot \mathrm{mol}^{-1} \cdot \AA^{-2}$. ABF force was gradually applied from 200 samples accumulated in a bin, and it fully reached its actual value at 400 and more samples (linear scaling between the first and second value). Total sampling achieved by the ABF/MWA simulation was $4.4 \mu \mathrm{s}$.

Final free energy surface was reconstructed from the calculated forces by Gaussian Process Regression (GPR). Optimisation of GPR hyperparameters was achieved by maximising the logarithm of the marginal likelihood. Trajectories were analysed by CATs ${ }^{24}$, and visualisation was done in $\mathrm{VMD}^{25}$ and Gnuplot. ${ }^{26}$ Reaction pathways were recovered as minimum free energy paths on the calculated free energy surface. 

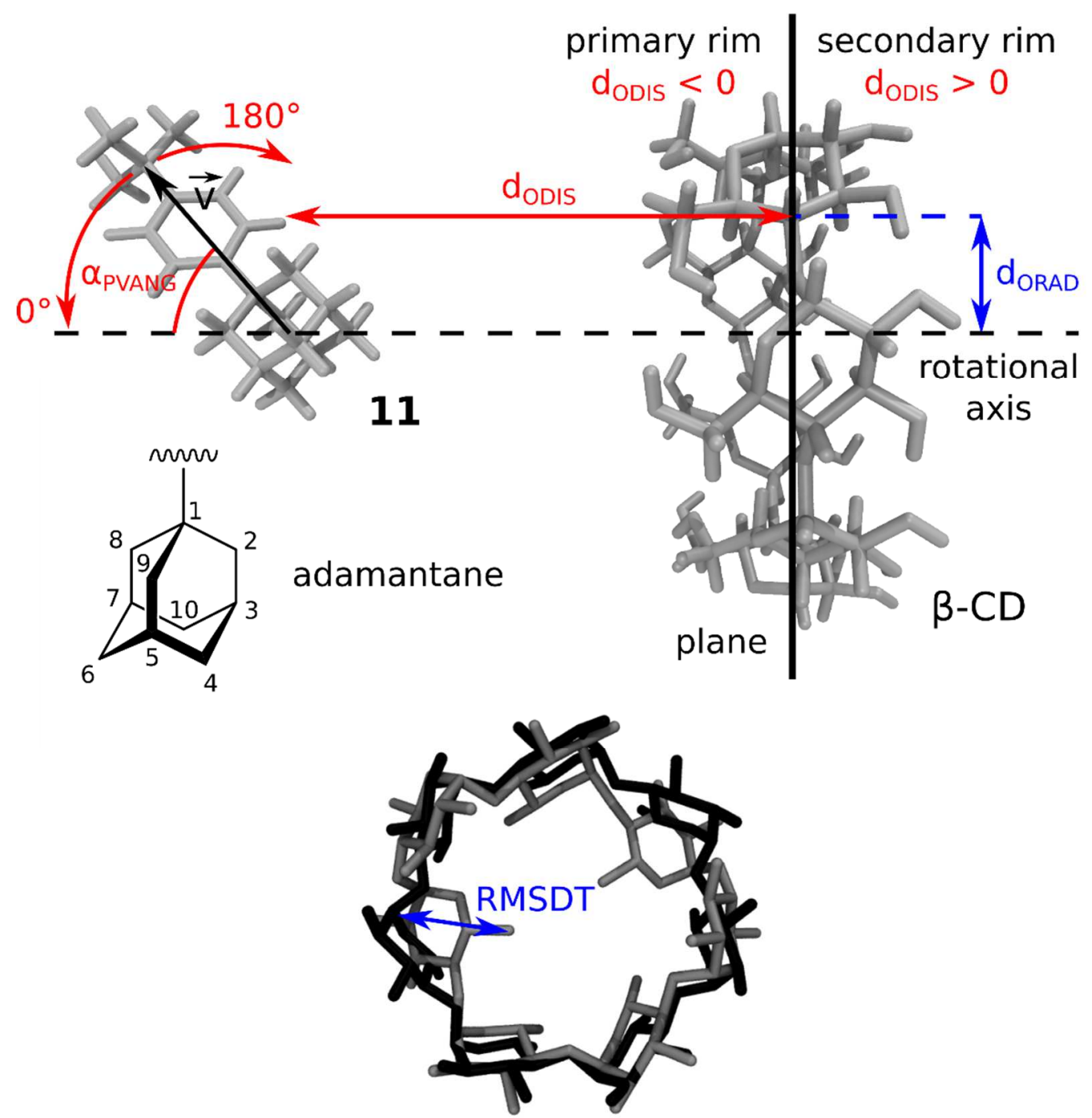

Figure S61. Collective variables (red) and restraints (blue) employed during biased molecular dynamics exploring binding modes of guest 11 into $\beta$-CD. The numbering of the adamantane moiety is provided for the definition of collective variables (see the text). 
Table S8. Parameters of stationary points found on the free energy surface (Figure 4A). Free energies are reported relative to NS.

\begin{tabular}{lcrrrrr} 
Free Energy Minimum & $\begin{array}{l}\text { Representative } \\
\text { structure }\end{array}$ & $d_{\mathrm{ODIS}}[\AA ⿻$ & $\alpha_{\mathrm{PVANG}}\left[{ }^{\circ}\right]$ & $\Delta G\left[\mathrm{~kJ} \cdot \mathrm{mol}^{-1}\right]$ \\
\hline NS & MD-ABF/NS.xyz & 2.8 & 130.6 & 0.0 & \pm & 0.0 \\
NP & MD-ABF/NP.xyz & -1.2 & 17.7 & 5.1 & \pm & 1.5 \\
IP & MD-ABF/IP.xyz & -5.7 & 95.4 & 27.2 & \pm & 1.6 \\
\hline Transition State & & & & & & \\
\hline T1P & MD-ABF/T1P.xyz & -4.8 & 72.8 & 38.1 & \pm & 1.7 \\
T2P & MD-ABF/T2P.xyz & -4.0 & 132.9 & 51.9 & \pm & 1.6 \\
T1S & MD-ABF/T1S.xyz & 3.9 & 55.7 & 41.0 & \pm & 1.2
\end{tabular}

${ }^{a}$ These structures represent the mutual position of interacting molecules (11 and $\left.\beta-C D\right)$ in the complex $11 @ \beta-C D$. They were randomly selected from ensembles of snapshots representing given free energy minima taken from biased ABF/MWA MD simulations. The water molecules and counterions were removed for clarity. The structures are available in the XYZ format in the attached ZIP archive. 


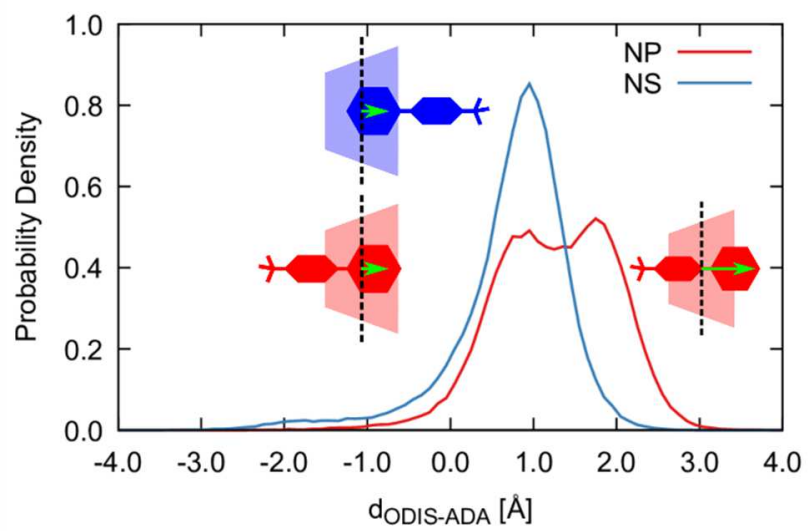

Figure S62. Distribution of oriented distance $d_{\mathrm{ODIS}-\mathrm{ADA}}$ between adamantyl of $\mathbf{1 1}$ and $\beta$-CD from unbiased molecular dynamics of NP and NS orientations. For NS, one adamantyl position with respect to $\beta$-CD was favoured, whereas NP displayed two positions. While the first one was comparable to the position of NS, the second one was shifted towards the secondary portal of $\beta$-CD. Oriented distance $d_{\text {ODIS-ADA }}$ focused only on adamantyl contrary to $d_{\text {ODIS }}$, which employed the centre of mass of all heavy atoms of $\mathbf{1 1}$ (Figure S60). 

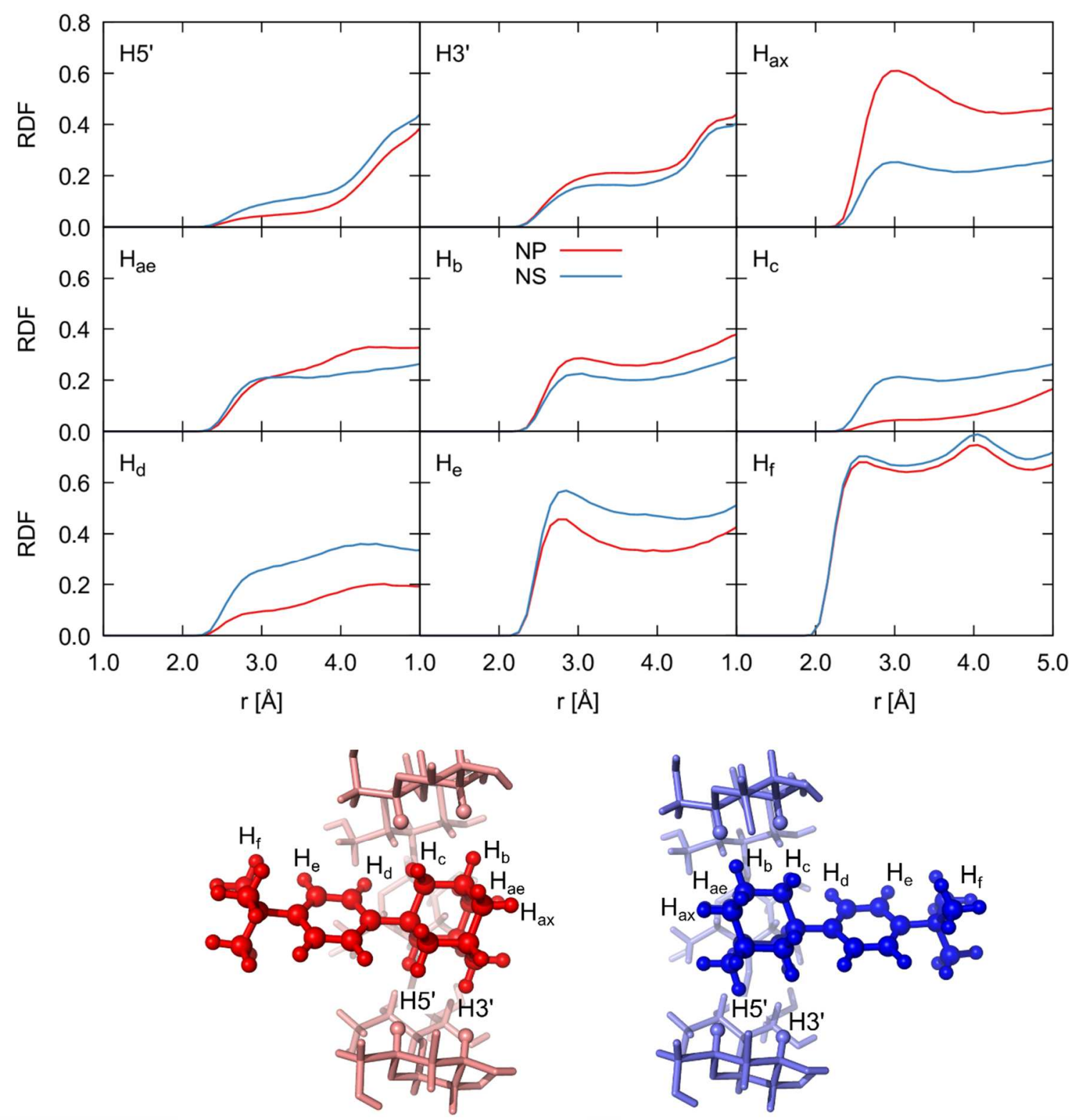

Figure S63 Radial distribution functions (RDF) calculated between protons of $11 @ \beta$-CD complex and oxygen atoms of water. The analysis shows the difference between solvation of NP and NS binding modes, which correlates with large differences between chemical shifts of NP and NS forms observed for $\mathrm{H}_{\mathrm{ax}}, \mathrm{H}_{\mathrm{c}}$, and $\mathrm{H}_{\mathrm{d}}$ atoms. 


\section{Quantum-chemical Calculations}

\section{Nucleus-Independent Chemical Shift (NICS)}

The input coordinates of benzene were optimised using the PBE0 density functional ${ }^{27}$ and the def2TZVPP basis set ${ }^{11,12}$ with D3BJ dispersion correction ${ }^{8,9}$ using the Gaussian 16.B1 software package. ${ }^{28}$ The resulting coordinates were used to compute the spatial magnetic shielding via the NICS approach. ${ }^{29}$ First, a three-dimensional grid of pseudo-atoms spaced $0.25 \AA$ apart was generated around the benzene. Then, the magnetic shielding was evaluated at the position of each pseudo-atom using the PBE0/6$311 \mathrm{G}^{* *}$ level of theory. ${ }^{30}$

Finally, we employed NICS data to rationalise chemical shifts observed for different binding modes. ${ }^{31}$ We focused on the through-space effect of benzene ring found in the structure of $\mathbf{1 1}$ on $\beta$-CD interior protons. Each snapshot from molecular dynamics of NP and NS was re-oriented such that the carbon atoms of the benzene ring were best fitted to the benzene used in the NICS calculation described above. Then, the NICS value was retrieved for H3' and H5' atoms of $\beta$-CD the from the volumetric map. These values were grouped according to topicity and averaged along the trajectory.

Dependence of NMR shielding of ligand 11 on its position with respect to $\beta$-CD

The rigid body scan shows changes of ${ }^{1} \mathrm{H}$ shielding of the ligand 11 upon encapsulation into the $\beta$ CD cavity. Structure of idealised $\beta-C D$ and geometry optimised ligand $\mathbf{1 1}$ were shifted mutually along the $z$-axis using the ligand $\mathrm{N}$ atom and the XY-plane of $\beta$-CD defined by the oxygen glycosidic atoms with $0.5 \AA$ step in the range $\sim 6-20 \AA$. The scanned distance was then transformed into $d_{\text {ODIS-ADA }}$ (Figure S61). The NMR shieldings of ligand hydrogen atoms were evaluated using B3LYP/def2-SVP( $\beta$ CD),def2-TZVPP(ligand)/SMD employing RI and RIJCOSX approximations and def2/J auxiliary basis set in Orca. For employed structure geometries, see Tables S9 and S10. 
Table S9. Structures employed in the rigid body scan of ligand $\mathbf{1 1}$ into the $\beta$-CD cavity from the NP side, their total energies at the B3LYP/def2-SVP( $\beta-C D)$,def2-TZVPP(ligand)/SMD, and value of geometrical parameter $\mathrm{d}_{\mathrm{ODIS}-\mathrm{ADA}}$.

\begin{tabular}{llr}
\hline Structure & Energy [a.u.] & dodIs-ADA [Å] \\
\hline QM-RigidScan/NP/geom_NP_01.xyz & -5063.933842 & 1.2 \\
QM-RigidScan/NP/geom_NP_02.xyz & -5063.936792 & 0.9 \\
QM-RigidScan/NP/geom_NP_03.xyz & -5063.934161 & 0.5 \\
QM-RigidScan/NP/geom_NP_04.xyz & -5063.935224 & -0.1 \\
QM-RigidScan/NP/geom_NP_05.xyz & -5063.937662 & -0.5 \\
QM-RigidScan/NP/geom_NP_06.xyz & -5063.936526 & -0.8 \\
QM-RigidScan/NP/geom_NP_07.xyz & -5063.932529 & -0.9 \\
QM-RigidScan/NP/geom_NP_08.xyz & -5063.931806 & -1.5 \\
QM-RigidScan/NP/geom_NP_09.xyz & -5063.936453 & -1.9 \\
QM-RigidScan/NP/geom_NP_10.xyz & -5063.943021 & -2.5 \\
QM-RigidScan/NP/geom_NP_11.xyz & -5063.947242 & -2.9 \\
QM-RigidScan/NP/geom_NP_12.xyz & -5063.947646 & -3.5 \\
QM-RigidScan/NP/geom_NP_13.xyz & -5063.948699 & -3.9 \\
QM-RigidScan/NP/geom_NP_14.xyz & -5063.948328 & -4.5 \\
QM-RigidScan/NP/geom_NP_15.xyz & -5063.947481 & -4.9 \\
QM-RigidScan/NP/geom_NP_16.xyz & -5063.947628 & -5.5 \\
QM-RigidScan/NP/geom_NP_17.xyz & -5063.948281 & -5.9 \\
QM-RigidScan/NP/geom_NP_18.xyz & -5063.948020 & -6.5 \\
QM-RigidScan/NP/geom_NP_19.xyz & -5063.947771 & -6.9 \\
QM-RigidScan/NP/geom_NP_20.xyz & -5063.947357 & -7.5 \\
\hline
\end{tabular}


Table S10. Structures employed in the rigid body scan of ligand $\mathbf{1 1}$ into the $\beta$-CD cavity from the NS side, their total energies at the B3LYP/def2-SVP( $\beta-C D)$,def2-TZVPP(ligand)/SMD, and value of geometrical parameter dODIS-ADA.

\begin{tabular}{llr}
\hline Structure & Energy [a.u.] & d $_{\text {ODIs-ADA [Å] }}$ \\
\hline QM-RigidScan/NS/geom_NS_01.xyz & -5063.936321 & -1.2 \\
QM-RigidScan/NS/geom_NS_02.xyz & -5063.933409 & -0.9 \\
QM-RigidScan/NS/geom_NS_03.xyz & -5063.928603 & -0.5 \\
QM-RigidScan/NS/geom_NS_04.xyz & -5063.928482 & 0.1 \\
QM-RigidScan/NS/geom_NS_05.xyz & -5063.933598 & 0.5 \\
QM-RigidScan/NS/geom_NS_06.xyz & -5063.939671 & 0.8 \\
QM-RigidScan/NS/geom_NS_07.xyz & -5063.941339 & 0.9 \\
QM-RigidScan/NS/geom_NS_08.xyz & -5063.940629 & 1.5 \\
QM-RigidScan/NS/geom_NS_09.xyz & -5063.941683 & 1.9 \\
QM-RigidScan/NS/geom_NS_10.xyz & -5063.942796 & 2.5 \\
QM-RigidScan/NS/geom_NS_11.xyz & -5063.943734 & 2.9 \\
QM-RigidScan/NS/geom_NS_12.xyz & -5063.944279 & 3.5 \\
QM-RigidScan/NS/geom_NS_13.xyz & -5063.944503 & 3.9 \\
QM-RigidScan/NS/geom_NS_14.xyz & -5063.939456 & 4.5 \\
QM-RigidScan/NS/geom_NS_15.xyz & -5063.939902 & 4.9 \\
QM-RigidScan/NS/geom_NS_16.xyz & -5063.945414 & 5.5 \\
QM-RigidScan/NS/geom_NS_17.xyz & -5063.946067 & 5.9 \\
QM-RigidScan/NS/geom_NS_18.xyz & -5063.947118 & 6.5 \\
QM-RigidScan/NS/geom_NS_19.xyz & -5063.947007 & 6.9 \\
QM-RigidScan/NS/geom_NS_20.xyz & -5063.946978 & 7.5 \\
\hline
\end{tabular}


${ }^{1}$ S. Moghaddam, C. Yang, M. V. Rekharsky, Y. H. Ko, K. Kim, Y. Inoue, M. K. Gilson J. Am. Chem. Soc. 2011, 133, 3570-3581.

${ }^{2}$ M. V. Rekharsky, Y. Inoue Chem. Rev. 1998, 98, 1875-1917.

${ }^{3}$ S. Grimme, J. Antony, S. Ehrlich, H. Krieg J. Chem. Phys. 2010, 132, 154104.

${ }^{4}$ S. Grimme, S. Ehrlich, L. Goerigk J. Comput. Chem. 2011, 32, 1456-1465.

${ }^{5}$ V. N. Staroverov, G. E. Scuseria, J. Tao, J. P. Perdew J. Chem. Phys. 2003, 119, 12129-12137.

${ }^{6}$ F. Weigend, R. Ahlrichs Phys. Chem. Chem. Phys. 2005, 7, 3297.

${ }^{7}$ F. Weigend Phys. Chem. Chem. Phys. 2006, 8, 1057.

${ }^{8}$ F. Neese Wiley Interdiscip. Rev.-Comput. Mol. Sci. 2012, 2, 73-78.

${ }^{9}$ M. J. Frisch, G. W. Trucks, H. B. Schlegel, G. E. Scuseria, M. A. Robb, J. R. Cheeseman, G. Scalmani, V. Barone, B. Mennucci, G. A. Petersson et al. Gaussian 09, Revision E.1; Gaussian, Inc.: Wallingford CT, 2009. ${ }^{10}$ C. I. Bayly, P. Cieplak, W. D. Cornell, P. A. Kollman J. Phys. Chem. 1993, 97, 10269-10280.

${ }^{11}$ J. Wang, W. Wang, P. A. Kollman J. Mol. Graph. Model. 2006, 25, 247-260.

${ }^{12}$ K. N. Kirschner, A. B. Yongye, S. M. Tschampel, J. González-Outeiriño, C. R. Daniels, B. L. Foley, R. J. Woods J. Comput. Chem. 2008, 29, 622-655.

${ }^{13}$ W. L. Jorgensen, J. Chandrasekhar, J. D. Madura, R. W. Impey, M. L. Klein J. Chem. Phys. 1983, 79, 926.

${ }^{14}$ H. Grubmuller Solvate 1.0; Ludwig-Maximilians-Universität München, 1996.

${ }^{15}$ I. S. Joung, T. E. Cheatham J. Phys. Chem. B 2008, 112, 9020-9041.

${ }^{16}$ D. A. Case, R. M. Betz, W. Botello-Smith, D. S. Cerutti, T. E. Cheatham III, T. A. Darden, R. E. Duke, T. J. Giese, H. Gohlke, A. W. Goetz et al. AMBER 16; University of California: San Francisco, 2016.

${ }^{17}$ T. Darden, D. York, L. Pedersen J. Chem. Phys. 1993, $98,10089$.

${ }^{18}$ D. Quigley, M. I. J. Probert J. Chem. Phys. 2004, 120, 11432.

${ }^{19}$ J.-P. Ryckaert, G. Ciccotti, H. J. C. Berendsen J. Comput. Phys. 1977, 23, 327-341.

${ }^{20}$ R. Salomon-Ferrer, A. W. Götz, D. Poole, S. Le Grand, R. C. Walker J. Chem. Theory Comput. 2013, 9 , 3878-3888.

${ }^{21}$ H. J. C. Berendsen, J. P. M. Postma, W. F. van Gunsteren, A. DiNola, J. R. Haak J. Chem. Phys. 1984, 81, 3684.

${ }^{22}$ J. Comer, J. C. Gumbart, J. Hénin, T. Lelièvre, A. Pohorille, C. Chipot J. Phys. Chem. B 2015, 119, 11291151.

${ }^{23}$ P. Kulhánek, J. Štěpán, M. Fuxreiter, L. Mones, Z. Střelcová, M. Petřek PMFLib - A Toolkit for Free Energy Calculations https://github.com/kulhanek/pmflib.

${ }^{24}$ P. Kulhánek, J. Štěpán, J. Ol’ha, M. Růžička, V. Illík CATs - Conversion and Analysis Tools https://github.com/kulhanek/cats.

${ }^{25}$ W. Humphrey, A. Dalke, K. Schulten J. Mol. Graph. 1996, 14, 33-38.

${ }^{26}$ The Gnuplot team (http://gnuplot.info). Gnuplot 5.0.

27 (a) J. P. Perdew, K. Burke, M. Ernzerhof Phys. Rev. Lett. 1996, 77, 3865-3868; (b) C. Adamo, V. Barone J. Chem. Phys. 1999, 110, 6158-6170.

${ }^{28}$ Gaussian 16, Revision B.01, M. J. Frisch, G. W. Trucks, H. B. Schlegel, G. E. Scuseria, M. A. Robb, J. R. Cheeseman, G. Scalmani, V. Barone, G. A. Petersson, H. Nakatsuji, X. Li, M. Caricato, A. V. Marenich, J. Bloino, B. G. Janesko, R. Gomperts, B. Mennucci, H. P. Hratchian, J. V. Ortiz, A. F. Izmaylov, J. L. Sonnenberg, D. Williams-Young, F. Ding, F. Lipparini, F. Egidi, J. Goings, B. Peng, A. Petrone, T. Henderson, D. Ranasinghe, V. G. Zakrzewski, J. Gao, N. Rega, G. Zheng, W. Liang, M. Hada, M. Ehara, K. Toyota, R. Fukuda, J. Hasegawa, M. Ishida, T. Nakajima, Y. Honda, O. Kitao, H. Nakai,

T. Vreven, K. Throssell, J. A. Montgomery, Jr., J. E. Peralta, F. Ogliaro, M. J. Bearpark, J. J. Heyd, E. N. Brothers, K. N. Kudin, V. N. Staroverov, T. A. Keith, R. Kobayashi, J. Normand, K. Raghavachari, A. P. Rendell, J. C. Burant, S. S. Iyengar, J. Tomasi, M. Cossi, J. M. Millam, M. Klene, C. Adamo, R. Cammi, J. W. Ochterski, R. L. Martin, K. Morokuma, O. Farkas, J. B. Foresman, and D. J. Fox, Gaussian, Inc., Wallingford CT, 2016.

${ }^{29}$ P. Schleyer, C. Maerker, A. Dransfeld, H. Jiao, J. R. van Eikema Hommes J. Am. Chem. Soc. 1996, 118, 6317-6318.

${ }^{30}$ R. Krishnan, J. S. Binkley, R. Seeger, J. A. Pople J. Chem. Phys. 1980, 72, 650-654.

${ }^{31}$ P. Branná, J. Černochová, M. Rouchal, P. Kulhánek, M. Babinský, R. Marek, M. Nečas, I. Kuřitka, R. Vícha J. Org. Chem. 2016, 81, 9595-9604. 